\title{
In vivo bone strain and finite element modeling of the mandible of Alligator mississippiensis
}

\author{
Laura B. Porro, ${ }^{1,2}$ Keith A. Metzger, ${ }^{3}$ Jose Iriarte-Diaz ${ }^{1}$ and Callum F. Ross ${ }^{1}$ \\ ${ }^{1}$ Department of Organismal Biology and Anatomy, University of Chicago, Chicago, IL, USA \\ ${ }^{2}$ School of Earth Sciences, University of Bristol, Bristol, UK \\ ${ }^{3}$ Department of Science Education, Hofstra North Shore - LIJ School of Medicine, Hempstead, NY, USA
}

\begin{abstract}
Forces experienced during feeding are thought to strongly influence the morphology of the vertebrate mandible; in vivo strain data are the most direct evidence for deformation of the mandible induced by these loading regimes. Although many studies have documented bone strains in the mammalian mandible, no information is available on strain magnitudes, orientations or patterns in the sauropsid lower jaw during feeding. Furthermore, strain gage experiments record the mechanical response of bone at a few locations, not across the entire mandible. In this paper, we present bone strain data recorded at various sites on the lower jaw of Alligator mississippiensis during in vivo feeding experiments. These data are used to understand how changes in loading regime associated with changes in bite location are related to changes in strain regime on the working and balancing sides of the mandible. Our results suggest that the working side mandible is bent dorsoventrally and twisted about its long-axis during biting, and the balancing side experiences primarily dorsoventral bending. Strain orientations are more variable on the working side than on the balancing side with changes in bite point and between experiments; the balancing side exhibits higher strain magnitudes. In the second part of this paper, we use principal strain orientations and magnitudes recorded in vivo to evaluate a finite element model of the alligator mandible. Our comparison demonstrates that strain orientations and mandibular deformation predicted by the model closely match in vivo results; however, absolute strain magnitudes are lower in the finite element model.
\end{abstract}

Key words: biomechanics; crocodilians; feeding; validation.

\section{Introduction}

Understanding the extent to which combinations of external forces (loading regimes) acting on the skeleton are associated with internal stress, strain and deformation regimes is fundamental in evaluating hypotheses regarding formfunction relationships and is an important objective of biomechanics, functional morphology and vertebrate paleontology (Bock \& Von Wahlert, 1965; Plotnick \& Baumiller, 2000; Schwenk, 2000). Moreover, obtaining such data from several taxa can be used to relate interspecific differences in skeletal morphology to differences in behavior, habitat or diet.

The vertebrate mandible is an important structure in which to investigate the association between loading regime and stress and strain regimes because of its role in

Correspondence

Laura B. Porro, Department of Organismal Biology and Anatomy, University of Chicago, 1027 East 57th Street, Chicago, IL 60637, USA. E: laura.porro@bristol.ac.uk

Accepted for publication 13 June 2013

Article published online 15 July 2013 feeding, during which it transmits forces to and from the organism and environment. Relationships between in vivo loading regimes, strain regimes and mandibular morphology have been examined in numerous mammalian taxa, including rabbits (Weijs \& De Jongh, 1977), pigs (Liu \& Herring, 2000; Herring et al. 2001), hyraxes (Lieberman et al. 2004), selenodont artiodactyls (Williams et al. 2008, 2009) and primates (Hylander, 1979b, 1981, 1984; Hylander et al. 1987, 1998), but we know of no published in vivo bone strain studies examining the sauropsid (Testudines + Squamates + Archosaurs) lower jaw. As a result, comparative hypotheses regarding the functional significance of variation in mandibular morphology across vertebrates are currently limited (but see Hylander \& Crompton, 1986). Additionally, numerous morphological features characteristic of sauropsids, including the presence of mandibular sutures, a mandibular fenestra, and lack of adductor musculature lateral to the mandibular ramus, are absent in mammals, making extrapolation of sauropsid mandibular function from mammalian studies tenuous (Weishampel, 1995). Documenting in vivo mechanical behavior of the Alligator mandible is a first step towards understanding 
sauropsid jaw biomechanics, and takes the study of feeding function in reptiles beyond the realm of theoretical analyses. Furthermore, the extreme bite forces reported in crocodilians (Erickson et al. 2003, 2012) and high strain magnitudes recorded from the Alligator cranium (Ross \& Metzger, 2004) suggest that feeding may exert particularly strong selective pressure on mandibular morphology in crocodilians.

Cranial mechanical behavior in various extant and extinct sauropsids has been modeled using different techniques (Busbey, 1995; Daniel \& McHenry, 2001; Metzger et al. 2005; McHenry et al. 2006; Rayfield et al. 2007; Pierce et al. 2008; Rayfield \& Milner, 2008; Moazen et al. 2009; Soons et al. 2010); in contrast, the sauropsid mandible has received little attention. Studies of sauropsid mandibular function include: free-body analyses of crocodilian, dinosaur and bird mandibles (Bock, 1966; Van Drongelen \& Dullemeijer, 1982; Molnar, 1998); beam modeling of plesiosaur, crocodilian and theropod dinosaur mandibles (Taylor, 1992; Therrien et al. 2005; Porro et al. 2011); photoelastic studies of bird mandibles (Bock \& Kummer, 1968) and finite element analysis (FEA) of the mandibles of Varanus, Alligator, several dinosaur taxa, and ostrich (Mazzetta et al. 2004; Moreno et al. 2008; Bell et al. 2009; Porro et al. 2011; Rayfield, 2011; Reed et al. 2011). FEA is a useful tool for understanding the mechanical behavior of geometrically and materially complex structures that cannot be adequately modeled using simpler methods. FEA also has advantages over strain gage experiments: strain can be calculated in places inaccessible to gages; strain can be observed throughout the entire structure rather than at a limited number of sites; and three-dimensional internal strains are recorded in addition to surface strains. Furthermore, FEA can be used to test the mechanical significance of anatomical features by generating hypothetical structures (Strait et al. 2007; Rayfield \& Milner, 2008) and revealing the mechanical behavior of extinct sauropsid skulls (Rayfield et al. 2001, 2007; Bell et al. 2009; Mazzetta et al. 2009).

Results generated by FEA should be evaluated against in vivo/in vitro strain data both to appraise the accuracy of model predictions (validation) and to determine how variability in input parameters impacts model results (sensitivity analyses). Validation studies comparing the behavior of finite element model (FEM) skulls with in vivolin vitro results have been largely confined to mammals (Verrue et al. 2001; Ross et al. 2005, 2011; Strait et al. 2005; Kupczik et al. 2007; Bright \& Rayfield, 2011; Panagiotopoulou et al. 2011). An FEM of the Alligator cranium is the only nonmammalian skull for which a validation study has been carried out (Metzger et al. 2005) and no model of a sauropsid mandible has yet been validated against in vivo strain data (see (Rayfield, 2011) comparing in vitro bone strains and FEA in the ostrich). Although previous studies have used FEA to predict overall mandibular deformation in the
Alligator mandible (Porro et al. 2011) and quantify model sensitivity to input parameters (Reed et al. 2011), it is unclear how well model predictions reflect reality without validation.

The goals of this study are: (i) to document patterns of in vivo bone strain in the Alligator mandible during biting, including variance in strain patterns at multiple gage sites during biting at different points along the tooth row on the working (biting) and balancing (non-biting) sides and calculation of the neutral axis of bending in a transverse section of the mandible; (ii) to use in vivo bone strain data to validate a high resolution FEM of the Alligator mandible; (iii) to use the FEM to predict overall patterns of deformation and strain in the Alligator mandible during biting at different points along the toothrow; and (iv) to compare Alligator mandibular strain regimes with those collected from mammals.

\section{Material and methods}

\section{In vivo bone strain recording and analysis}

\section{Subjects}

Three sub-adult American alligators (Alligator mississippiensis; head length $12.3,20.5$, and $17.5 \mathrm{~cm}$ ) were used in five separate experiments. Animals were individually housed in large enclosures with wet and dry areas on a 12-h light/dark cycle and fed mice, chicken and fish three times per week. Environmental temperature ranged from $28{ }^{\circ} \mathrm{C}$ during the day to $20^{\circ} \mathrm{C}$ at night and water was kept at a constant temperature of $27{ }^{\circ} \mathrm{C}$ using a submersible heater. Animals were housed in the Stony Brook University Division of Laboratory Animal Resources in accordance with the National Institutes of Health Guidelines for the Care and Use of Laboratory Animals. All experimental procedures were approved by the Stony Brook University Institutional Animal Care and Use Committee.

\section{Bone strain data collection}

During experiments, stacked delta rosette strain gages (SA-06030WY-120; Micromeasurements, Raleigh, NC) or rectangular rosette strain gages (FRA 1-11-1L; Texas Measurements, Inc., College Station, TX) were wired, insulated, and gas-sterilized using previously described procedures (Ross, 2001). Following anesthesia with $2 \%$ isofluorane administered in oxygen through an intubation tube, $1 \mathrm{~cm}^{2}$ of skin overlying the gage sites was removed, the periosteum elevated, the bone degreased with chloroform, and the gage bonded to the surface of the bone using cyanoacrylate adhesive. Gages were placed in multiple locations during five separate experiments, including the dorsolateral, lateral and ventral surfaces of the dentary, ventral and medial surfaces of the splenial, and the angular (Table 1, Fig. 1). Gage lead wires were epoxied and sutured to the skin overlying the mandible to provide strain relief and secured to the back of the animal with veterinary tape. Following surgery, all animals were radiographed to obtain a permanent record of strain gage locations.

Animals recovered from surgery for at least $2 \mathrm{~h}$ before strains were recorded while the animal bit unilaterally on steel bite plates covered with several layers of surgical tape. The upper layer was replaced after each bite so that bite position was accurately 
Table 1 Experimental summary including strain gage locations by experiment. For gages with nearly identical placement in different experiments, only one site was analyzed in the FEM.

\begin{tabular}{|c|c|c|c|c|}
\hline $\begin{array}{l}\text { Experiment } \\
\text { ID number }\end{array}$ & $\begin{array}{l}\text { Animal } \\
\text { ID }\end{array}$ & $\begin{array}{l}\text { Mandible } \\
\text { side }\end{array}$ & Gage location & $\begin{array}{l}\text { FEM gage } \\
\text { ID number }\end{array}$ \\
\hline \multirow[t]{3}{*}{56} & 1 & Right & $\begin{array}{l}\text { Anterior ventral } \\
\text { dentary }\end{array}$ & 1 \\
\hline & & & $\begin{array}{l}\text { Posterior ventral } \\
\text { dentary }\end{array}$ & 2 \\
\hline & & & Ventral angular & 3 \\
\hline \multirow[t]{3}{*}{75} & 1 & Left & Lateral dentary & 4 \\
\hline & & & Ventral dentary & 5 \\
\hline & & & Medial splenial & 6 \\
\hline \multirow[t]{4}{*}{99} & 2 & Right & $\begin{array}{l}\text { Dorsolateral } \\
\text { dentary }\end{array}$ & 7 \\
\hline & & & Lateral dentary & 4 \\
\hline & & & Ventral dentary & 5 \\
\hline & & & Medial splenial & 6 \\
\hline \multirow[t]{5}{*}{102} & 3 & Left & $\begin{array}{l}\text { Dorsolateral } \\
\text { dentary }\end{array}$ & 8 \\
\hline & & & Lateral dentary & 9 \\
\hline & & & Ventral dentary & 10 \\
\hline & & & Ventral splenial & 11 \\
\hline & & & Medial splenial & 12 \\
\hline \multirow[t]{4}{*}{103} & 3 & Right & $\begin{array}{l}\text { Dorsolateral } \\
\text { dentary }\end{array}$ & 7 \\
\hline & & & Lateral dentary & 13 \\
\hline & & & Ventral dentary & 1 \\
\hline & & & Medial splenial & 14 \\
\hline
\end{tabular}

recorded using tooth impressions. To investigate variance in strain patterns with changes in bite point, the location of each recorded bite was assigned to one of 10 equal-sized regions of the mandible: left or right anterior, anterior/middle, middle, middle/posterior, and posterior regions (Fig. 1) (additionally, strains were recorded during biting at the midline anterior end of the jaws; results for these bites are presented in Tables 2-6 but not discussed). Biting side and location as well as the presence of any unusual activity (shake, attempted twisting) were recorded on a data sheet; data from these activities were excluded from the present study.

Voltage changes in the gages were conditioned and amplified on Vishay 2100 bridge-amplifiers. Data were acquired at $1 \mathrm{kHz}$ through a National Instruments DAQ board run by MIDAS data acquisition software package (Xcitex, Cambridge, MA) and saved to a PC.

\section{In vivo bone strain data analysis}

In vivo strain data were filtered and processed in IGOR PRO 4.0 (WaveMetrics, Inc., Lake Oswego, OR) using custom-written software. The strain data (strain is a dimensionless unit, $\varepsilon$, that represents change in length over original length or $\Delta \mathrm{L} / \mathrm{L}$ ) were converted to microstrain $\left(\mu \varepsilon\right.$, which are equal to $1 \times 10^{-6}$ inches per inch or $\mathrm{mm}$ per $\mathrm{mm}$ ) using calibration files produced during the recording sessions. The magnitude of maximum $\left(\varepsilon_{1}\right)$ and minimum $\left(\varepsilon_{2}\right)$ principal strains were calculated (Hibbeler, 2000) (Tables 2-6). Maximum principal strain $\left(\varepsilon_{1}\right)$ is usually the largest tensile strain value, and the minimum principal strain is usually the largest compressive strain value $\left(\varepsilon_{2}\right)$. The ratios of maximum to minimum principal strains $\left(\left|\varepsilon_{1} / \varepsilon_{2}\right|\right)$ were calculated (Tables 2-6) as was shear strain $(\gamma)$, which is equal to $\left|\varepsilon_{1}-\varepsilon_{2}\right|$. The orientations of the maximum principal strains $\left(\varepsilon_{1}\right)$ were also calculated (Tables 2-6). For consistency, $\varepsilon_{1}$ orientations for in vivo experiments and the FEM (as presented in tables and vector plots, as well as those used in statistical analyses) are calculated for a right mandible. The orientation of $\varepsilon_{1}$ in lateral, medial and ventral views were calculated relative to a reference axis aligned between the most anterior point of the external mandibular fenestra to the posterior base of the sixth dentary tooth; the reference plane was defined by this axis and another line parallel to either the ventral (for medial and lateral views) or medial (for ventral view) border of the mandible (Fig. 1). Converting strain orientations to this common reference axis enables comparisons between different experiments and between in vivo and FEM data. By convention, positive values are those rotated counterclockwise from the reference axis (vectors rotated clockwise from the axis are negative) when viewed from the right (for medial and lateral views) or from below (for ventral view). Custom software in IGOR PRO 4.0 was used to convert strain orientations and magnitudes to vectors within polar coordinates. Vector plots (Figs 2 and 3), in which the relative magnitudes and orientations of $\varepsilon_{1}$ for all gage sites during all recorded bites are displayed, were created using ADOBE ILLUSTRATOR CS 5.1 (Adobe System Incorporated, San Jose, CA).

Data from experiments in which three gages were placed around the circumference of the lower jaw allow normal strain distribution to be reconstructed at the section of the gages. From this distribution, maximum and minimum normal strains for the cross-section can be calculated (Rybicki et al. 1977; Demes et al. 2001; Demes, 2007). This gives an indication of the maximum tensile and compressive strains experienced in the section and allows the orientation of the neutral axis within the cross-section to be determined. The orientation of the neutral axis was calculated using data from four experiments $(75,99,102$ and 103) in which there were three or more gages placed around the circumference of the mandible in a coronal plane. To calculate the orientation of the neutral axis of bending, normal strains (strains normal to the plane of the crosssection) were calculated from peak principal strains recorded during each bite. Orientation of the neutral axis is reliant upon both normal strains and bone cross-sectional geometry (Rybicki et al. 1977; Carter et al. 1981; Demes, 2007). The orientation of the neutral axis was determined using the formula:

$\tan =\alpha=\left(I_{\max } / I_{\min }\right) / \tan \theta$

where $\alpha=$ angle of the neutral axis with maximum principal axis, $\theta=$ angle of external bending direction with maximum principal axis, and $I_{\max , \min }=$ maximum and minimum principal moments of cross-section (Hibbeler, 2000). Cross-sectional geometry of the mandible was determined from postmortem CT scans of the specimens. Normal strains were calculated assuming that material properties are homogeneous through the cross-section and that sutures do not dissipate or reorient strains. Calculations of normal strains and neutral axis were conducted using a custom macro for IGOR PRO provided by B. Demes, Stony Brook University.

\section{Finite element analysis}

\section{Model construction}

Strain data collected in vivo were compared with predictions from a high-resolution FEM of the Alligator mandible; additionally, the FEM was used to relate the in vivo strain data to global strain patterns and deformation throughout the Alligator mandible. The FEM described here has been used previously to understand the 

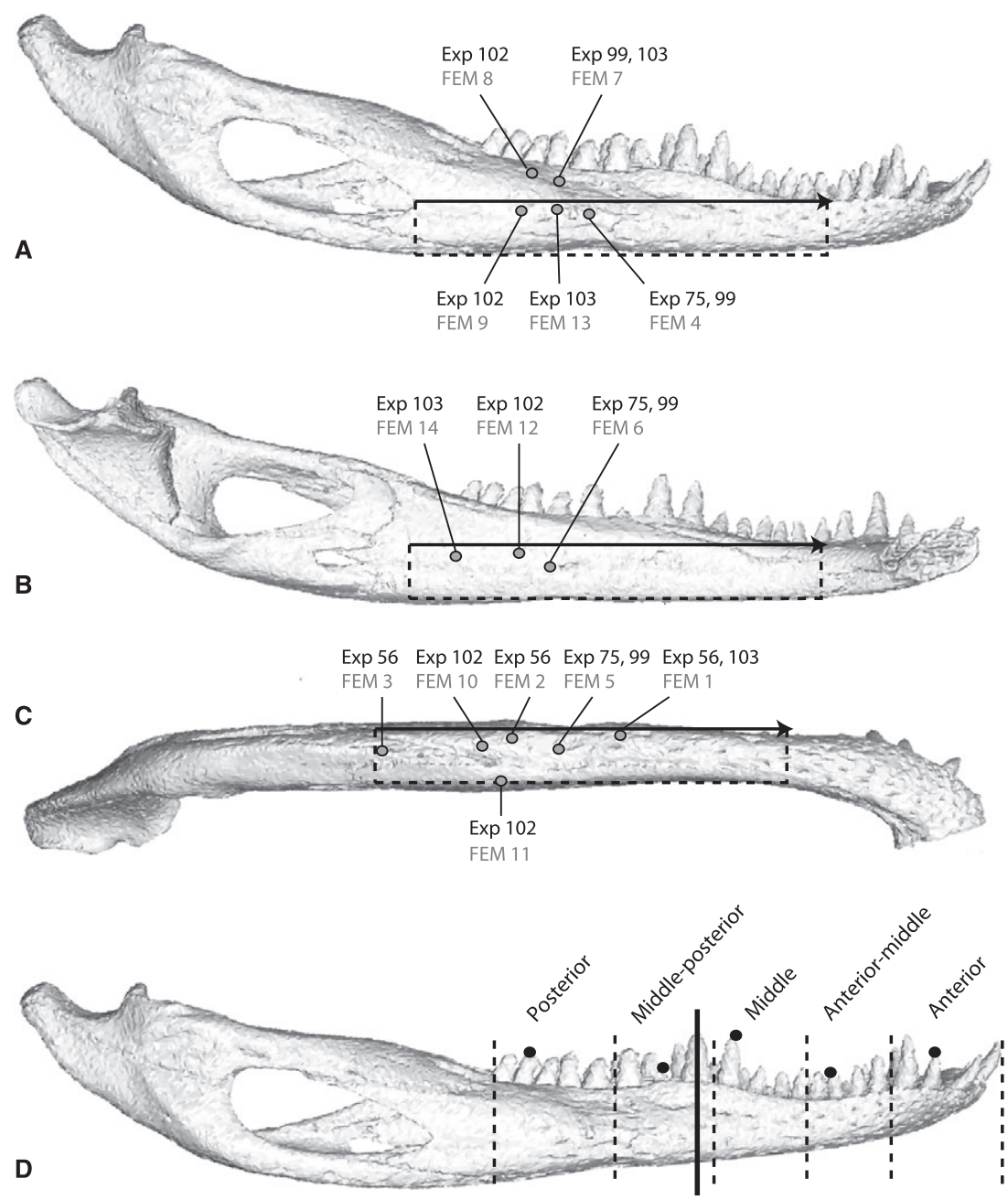

Fig. 1 Gage locations, reference axes and bite points. The mandible of the Alligator in lateral (A), medial (B) and ventral (C) views illustrating the locations of experimental strain gages (black text) and equivalent areas in the FEM (finite element model) (gray text). The reference axis used to standardize strain orientations across all experiments and the FEM is shown in all three views as a black arrow from the anterior point of the external mandibular fenestra to the posterior base of the 6th dentary tooth; strains rotated counterclockwise from this axis are positive, those rotated clockwise are negative. Dotted lines in $A, B$ and $C$ indicate the reference planes. Bite locations during in vivo experiments (dotted lines) and constraints in the FEM (black dots) are shown in (D). The solid black line in (D) indicates the section used to determine axis of bending in the FEM. impact of material properties on mandible deformation (Reed et al. 2011) and has been compared with results from simpler beam modeling and free-body analyses (Porro et al. 2011).

The preserved head (mandibular length of $189 \mathrm{~mm}$ ) of one of the individuals used in our experiments was $\mathrm{CT}$ scanned at Stony Brook University Medical Center using a GE LightSpeed 16 CT scanner at $100 \mathrm{kV}$ per $70 \mathrm{~mA}$ to produce 645 coronal slices with a slice thickness of $0.31 \mathrm{~mm}$ and a resolution of $0.25 \mathrm{~mm}$ per pixel. Scans were segmented in AMIRA 5.2.2 (Visage Imaging GmbH, Berlin, Germany) to generate a 3D surface model in which individual mandibular bones, sutures, periodontal ligament, and teeth were treated as separate parts; the cranium, including all bones and teeth, was segmented as a single part. The surface model was meshed with linear tetrahedra in the finite element software package STRAND7 2.4.1 (Strand7 Pty Ltd, Sydney, Australia) and the jaws of the model set at a gape angle of $30^{\circ}$.

The attachment sites of 16 jaw elevator muscles (the superficial, medial and deep portions of the external adductor; superficial and deep pseudotemporalis; posterior adductor; and dorsal and ventral portions of the pterygoideus on both sides of the head), and depressor mandibulae, were mapped onto the skull using information from dissections and the literature (Holliday \& Witmer, 2007). The reduced physiological cross-sectional area (PCSA), accounting for muscle mass, pinnation, and fiber and sarcomere lengths, was determined for each muscle (Porro et al. 2011); reduced PCSA was multiplied by $300 \mathrm{kN} \mathrm{m}^{-2}$ to yield the maximum contractile force generated by each muscle (Sinclair \& Alexander, 1987). Muscle forces were loaded onto the FEM using the VISUAL BASIC SCript BONELOAD (Grosse et al. 2007) which accounts for tensile, tangential and normal traction loads due to muscles wrapping around bone surfaces. The model was constrained at three nodes aligned mediolaterally across the joint surfaces of the quadrates (cranium) and articulars (mandible), preventing rigid body motion and generating joint reaction forces. Additionally, the tip of a single right dentary tooth (and its closest opposing premaxillary or maxillary tooth) was fixed to generate bite force. Five loading conditions were analyzed: anterior (3rd tooth), anterior-middle (7th tooth), middle (12th tooth), middle-posterior (14th tooth), and posterior (19th tooth) bite points, corresponding to similar areas in in vivo experiments. In all cases, the right side of the FEM was the working side and the left side was the balancing side. A single node was constrained on the medial surface of each mandible (opposite its contact with the pterygoid flange) to restrict medial bending and torsion of the mandibular ramus (the importance of this constraint is described in Porro et al. 2011).

Alligator mandibular bone properties applied to the FEM were obtained from an individual similar in size to the animals used in our experiment (Zapata et al. 2010; Porro et al. 2011). Mandibular sutures and periodontal ligament were assigned a density and Poisson's ratio reflecting the average reported in the literature (Currey, 
Table 2 Descriptive statistics for principal $\left(\varepsilon_{1}\right.$ and $\left.\varepsilon_{2}\right)$ and $\left|\left(\varepsilon_{1} / \varepsilon_{2}\right)\right|$ strain magnitude and $\varepsilon_{1}$ orientation for Experiment 56.

\begin{tabular}{|c|c|c|c|c|c|c|c|c|c|c|c|c|c|}
\hline \multirow{2}{*}{$\begin{array}{l}\text { Gauge } \\
\text { location }\end{array}$} & \multirow[b]{2}{*}{ Bite side } & \multirow{2}{*}{$\begin{array}{l}\text { Bite } \\
\text { position }\end{array}$} & \multirow[b]{2}{*}{$n$} & \multicolumn{3}{|c|}{$\varepsilon_{1}$} & \multicolumn{3}{|c|}{$\varepsilon_{2}$} & \multicolumn{2}{|c|}{$\varepsilon_{1} / \varepsilon_{2}$ ratio } & \multicolumn{2}{|c|}{$\begin{array}{c}\varepsilon_{1} \\
\text { orientation }\end{array}$} \\
\hline & & & & Mean & SD & Max. & Mean & SD & Max. & Mean & SD & Mean & SD \\
\hline \multirow{9}{*}{$\begin{array}{l}\text { Right } \\
\text { ventral } \\
\text { angular }\end{array}$} & Left (BS) & a & 4 & 71 & 45 & 122 & -120 & 49 & -148 & 0.61 & 0.27 & 113 & 33 \\
\hline & & $p$ & 5 & 56 & 21 & 88 & -129 & 41 & -183 & 0.48 & 0.25 & 101 & 28 \\
\hline & All left & & 9 & 63 & 34 & 122 & -125 & 42 & -183 & 0.54 & 0.25 & 107 & 28 \\
\hline & Right (WS) & $\mathrm{a} / \mathrm{m}$ & 2 & 35 & 31 & 57 & -205 & 158 & -317 & 0.16 & 0.03 & 66 & 3 \\
\hline & & $\mathrm{m}$ & 4 & 15 & 10 & 26 & -178 & 27 & -202 & 0.08 & 0.06 & 73 & 13 \\
\hline & & $m / p$ & 1 & 3 & & 3 & -273 & & & 0.01 & & 72 & \\
\hline & & $p$ & 6 & 14 & 4 & 20 & -206 & 61 & -314 & 0.07 & 0.03 & 66 & 6 \\
\hline & All right & & 13 & 17 & 14 & 57 & -202 & 67 & -314 & 0.09 & 0.05 & 69 & 9 \\
\hline & All bites & & 22 & 34 & 31 & 122 & -163 & 70 & -317 & 0.27 & 0.27 & 85 & 27 \\
\hline \multirow{10}{*}{$\begin{array}{l}\text { Right } \\
\text { anterior } \\
\text { dentary }\end{array}$} & Midline & & 2 & 426 & 193 & 563 & -648 & 354 & -899 & 0.68 & 0.07 & 110 & 7 \\
\hline & Left (BS) & a & 4 & 779 & 291 & 1003 & -1076 & 476 & -1487 & 0.75 & 0.09 & -1 & 1 \\
\hline & & $p$ & 6 & 457 & 149 & 703 & -568 & 230 & -962 & 0.82 & 0.05 & 6 & 4 \\
\hline & All left & & 10 & 600 & 257 & 1003 & -794 & 403 & -1487 & 0.79 & 0.07 & 3 & 4 \\
\hline & Right (WS) & $a / m$ & 2 & 782 & 117 & 865 & -570 & 53 & -608 & 1.37 & 0.08 & -25 & 2 \\
\hline & & $\mathrm{m}$ & 4 & 816 & 319 & 1244 & -908 & 426 & -1427 & 0.94 & 0.13 & -40 & 11 \\
\hline & & $m / p$ & 1 & 952 & & 952 & -819 & & -819 & 1.16 & & -29 & \\
\hline & & $p$ & 6 & 543 & 165 & 830 & -443 & 156 & -744 & 1.24 & 0.17 & -21 & 3 \\
\hline & All right & & 13 & 695 & 247 & 1244 & -634 & 320 & -1427 & 1.16 & 0.21 & -28 & 10 \\
\hline & All bites & & 22 & 637 & 254 & 1244 & -695 & 359 & -1487 & 0.98 & 0.26 & 0 & 53 \\
\hline \multirow{10}{*}{$\begin{array}{l}\text { Right } \\
\text { posterior } \\
\text { dentary }\end{array}$} & Midline & & 2 & 37 & 28 & 56 & -81 & 12 & -89 & 0.43 & 0.28 & -33 & 8 \\
\hline & Left (BS) & a & 4 & 125 & 45 & 182 & -77 & 29 & -105 & 1.65 & 0.16 & 12 & 6 \\
\hline & & $p$ & 6 & 118 & 30 & 142 & -95 & 29 & -140 & 1.36 & 0.51 & 20 & 24 \\
\hline & All left & & 10 & 121 & 47 & 182 & -87 & 26 & -140 & 1.49 & 0.4 & 17 & 19 \\
\hline & Right (WS) & $\mathrm{a} / \mathrm{m}$ & 2 & 54 & 16 & 65 & -47 & 30 & -68 & 1.57 & 1.33 & -12 & 14 \\
\hline & & $\mathrm{m}$ & 4 & 176 & 61 & 218 & -209 & 73 & -281 & 0.84 & 0.09 & -27 & 2 \\
\hline & & $m / p$ & 1 & 139 & & & -226 & & -226 & 0.62 & & -25 & \\
\hline & & $\mathrm{p}$ & 6 & 159 & 62 & 238 & -229 & 118 & -318 & 0.76 & 0.15 & -29 & 1 \\
\hline & All right & & 13 & 147 & 66 & 238 & -195 & 107 & -318 & 0.9 & 0.5 & -26 & 8 \\
\hline & All bites & & 22 & 128 & 61 & 238 & -145 & 97 & -318 & 1.08 & 0.56 & -13 & 54 \\
\hline
\end{tabular}

a, anterior; $a / m$, anterior/middle; $\mathrm{m}$, middle; $\mathrm{m} / \mathrm{p}$, middle/posterior; $\mathrm{p}$, posterior.

2002; Kupczik et al. 2007). Material properties for bone were anisotropic: $X$ refers to the mediolateral axis, $Y$ to the dorsoventral axis, and $Z$ to the anteroposterior axis of the mandible. The following properties were applied to the model: bone [density of $1662.8 \mathrm{~kg} \mathrm{~m}^{-3}$; elastic moduli of $8.1 \mathrm{GPA}(\mathrm{X}), 9.26 \mathrm{GPA}(\mathrm{Y})$ and 19.71 GPA (Z); shear moduli of 3.17 GPA (XY), 4.45 GPA (XZ), and 5.51 GPA (YZ); Poisson's ratios of $0.38(\mathrm{XY}), 0.08$ (XZ), and 0.15 (YZ)]; teeth (density of $2076 \mathrm{~kg} \mathrm{~m}^{-3}$; elastic modulus of $21 \mathrm{GPA}$; Poisson's ratio of 0.31 ); sutures and periodontal ligament (density of $1200 \mathrm{~kg} \mathrm{~m}^{-3}$; elastic modulus of 0.09 GPA; Poisson's ratio of 0.3). The FEM was solved using the linear static solver in STRAND7.

\section{In silico strain data extraction}

Prior to analysis, bricks on the surface of the FEM most closely corresponding to gage sites in the experiments were identified on both the working (right) and balancing (left) sides. The threedimensional 2 nd-order strain tensor of each brick within these sites were exported from STRAND7 to MATLAB (MathWorks, Nantick, MA) where custom-written code (Ross et al. 2011) was used to calculate the magnitude and orientation of the maximum and minimum principal surface strains in the FEM (Table 7). The orientations of the $\varepsilon_{1}$ surface strains were transformed relative to the reference axis described above using MATLAB, and strain orientations and magnitudes converted to vectors within polar coordinates in IGOR PRO 4.0, making $\varepsilon_{1}$ orientations from the FEM and in vivo experiments directly comparable (Table 7). Vectors representing the mean $\varepsilon_{1}$ orientation of all bricks within a gage site during anterior and posterior biting are superimposed over in vivo data in vector plots (Figs 2 and 3). Additionally, the ratio of maximum to minimum principal strains $\left(\left|\varepsilon_{1} / \varepsilon_{2}\right|\right)$ and maximum shear strain $(\gamma$-max) were calculated for FEM gage sites.

To determine the neutral axis of bending in the FEM, crosssections of the working and balancing sides at the level of the 13th dentary tooth were analyzed for strain perpendicular to the section. For each bite point, contour plots were used to determine the orientation of the neutral axis and the maximum and minimum principal strains within the section.

\section{In vivo and FEM bone strain data statistical analyses}

To quantify the effect of bite point on strain in the mandible, data were split into the 10 toothrow regions described above. On all data tables for in vivo experiments (Tables 2-6), missing data indicate that no strains were recorded for a particular bite position. 
Table 3 Descriptive statistics for principal $\left(\varepsilon_{1}\right.$ and $\left.\varepsilon_{2}\right)$ and $\left|\left(\varepsilon_{1} / \varepsilon_{2}\right)\right|$ strain magnitude and $\varepsilon_{1}$ orientation for experiment 75 .

\begin{tabular}{|c|c|c|c|c|c|c|c|c|c|c|c|c|c|}
\hline \multirow{2}{*}{$\begin{array}{l}\text { Gauge } \\
\text { location }\end{array}$} & \multirow[b]{2}{*}{ Bite side } & \multirow{2}{*}{$\begin{array}{c}\text { Bite } \\
\text { position }\end{array}$} & \multirow[b]{2}{*}{$n$} & \multicolumn{3}{|c|}{$\varepsilon_{1}$} & \multicolumn{3}{|c|}{$\varepsilon_{2}$} & \multicolumn{2}{|c|}{$\varepsilon_{1} / \varepsilon_{2}$ Ratio } & \multicolumn{2}{|c|}{$\begin{array}{c}\varepsilon_{1} \\
\text { Orientation }\end{array}$} \\
\hline & & & & Mean & SD & Max & Mean & SD & Max & Mean & SD & Mean & SD \\
\hline \multirow{12}{*}{$\begin{array}{l}\text { Left } \\
\text { lateral } \\
\text { dentary }\end{array}$} & Left (WS) & $\mathrm{a} / \mathrm{m}$ & 4 & 1098 & 420 & 1434 & -1131 & 449 & -1389 & 0.98 & 0.08 & -37 & 6 \\
\hline & & $\mathrm{m}$ & 6 & 530 & 271 & 848 & -387 & 321 & -961 & 1.66 & 0.44 & -29 & 12 \\
\hline & & $\mathrm{m} / \mathrm{p}$ & 12 & 1379 & 976 & 2846 & -976 & 786 & -2006 & 1.88 & 0.85 & -35 & 9 \\
\hline & & $\mathrm{p}$ & 8 & 1006 & 382 & 1386 & -615 & 283 & -1003 & 1.79 & 0.45 & -41 & 8 \\
\hline & All left & & 30 & 1073 & 727 & 2846 & -783 & 604 & -2006 & 1.69 & 0.67 & -36 & 9 \\
\hline & Right (BS) & a & 4 & 648 & 90 & 731 & -453 & 58 & -492 & 1.43 & 0.04 & 31 & 2 \\
\hline & & $\mathrm{a} / \mathrm{m}$ & 3 & 1328 & 203 & 1543 & -983 & 179 & -1172 & 1.36 & 0.04 & 33 & 1 \\
\hline & & $\mathrm{m}$ & 19 & 1492 & 290 & 1868 & -1157 & 248 & -1481 & 1.3 & 0.04 & 34 & 1 \\
\hline & & $\mathrm{m} / \mathrm{p}$ & 7 & 946 & 508 & 1728 & -714 & 427 & -1388 & 1.37 & 0.12 & 33 & 3 \\
\hline & & $p$ & 15 & 1191 & 446 & 1714 & -893 & 352 & -1325 & 1.35 & 0.07 & 32 & 2 \\
\hline & All right & & 48 & 1238 & 443 & 1868 & -941 & 365 & -1481 & 1.34 & 0.07 & 33 & 2 \\
\hline & All bites & & 78 & 1174 & 571 & 2846 & -880 & 474 & -2006 & 1.48 & 0.45 & 2 & 34 \\
\hline \multirow{12}{*}{$\begin{array}{l}\text { Left } \\
\text { ventral } \\
\text { dentary }\end{array}$} & Left (WS) & $\mathrm{a} / \mathrm{m}$ & 4 & 924 & 488 & 1382 & -1362 & 470 & -1632 & 0.63 & 0.22 & -51 & 3 \\
\hline & & $\mathrm{m}$ & 6 & 623 & 347 & 1118 & -420 & 388 & -1098 & 2.26 & 1.04 & -37 & 10 \\
\hline & & $\mathrm{m} / \mathrm{p}$ & 12 & 1930 & 1261 & 4016 & -1225 & 888 & -2714 & 1.9 & 0.59 & -29 & 7 \\
\hline & & $p$ & 6 & 2639 & 1027 & 3538 & -1569 & 687 & -2133 & 1.76 & 0.24 & -21 & 1 \\
\hline & All left & & 30 & 1723 & 1221 & 4016 & -1174 & 793 & -2713 & 1.76 & 0.75 & -32 & 11 \\
\hline & Right (BS) & $a$ & 4 & 839 & 135 & 976 & -1181 & 213 & -1419 & 0.71 & 0.02 & 67 & 2 \\
\hline & & $a / m$ & 3 & 1759 & 300 & 2064 & -2404 & 399 & -2791 & 0.73 & 0.01 & 61 & 0 \\
\hline & & $\mathrm{m}$ & 19 & 2017 & 476 & 2530 & -2757 & 644 & -3405 & 0.73 & 0.01 & 61 & 1 \\
\hline & & $\mathrm{m} / \mathrm{p}$ & 7 & 1216 & 742 & 2311 & -1661 & 1015 & -3113 & 0.74 & 0.02 & 61 & 1 \\
\hline & & $p$ & 15 & 1584 & 650 & 2379 & -2204 & 908 & -3307 & 0.72 & 0.02 & 62 & 0 \\
\hline & All right & & 48 & 1651 & 655 & 2530 & -2271 & 894 & -3405 & 0.73 & 0.01 & 62 & 1 \\
\hline & All bites & & 78 & 1679 & 908 & 4016 & -1849 & 1007 & -3405 & 1.13 & 0.69 & 19 & 43 \\
\hline \multirow{12}{*}{$\begin{array}{l}\text { Left } \\
\text { medial } \\
\text { splenial }\end{array}$} & Left (WS) & $a / m$ & 4 & 336 & 181 & 532 & -724 & 422 & -1131 & 0.48 & 0.04 & 110 & 4 \\
\hline & & $m$ & 6 & 198 & 85 & 264 & -410 & 186 & -595 & 0.5 & 0.08 & 113 & 2 \\
\hline & & $\mathrm{m} / \mathrm{p}$ & 12 & 242 & 132 & 454 & -541 & 302 & -949 & 0.47 & 0.07 & 115 & 3 \\
\hline & & $p$ & 8 & 252 & 52 & 332 & -552 & 130 & -708 & 0.46 & 0.06 & 113 & 2 \\
\hline & All left & & 30 & 249 & 116 & 532 & -542 & 267 & -1131 & 0.47 & 0.07 & 114 & 3 \\
\hline & Right (BS) & $a$ & 4 & 112 & 26 & 139 & -199 & 50 & -246 & 0.57 & 0.04 & 113 & 2 \\
\hline & & $a / m$ & 3 & 96 & 29 & 127 & -182 & 58 & -243 & 0.53 & 0.01 & 112 & 1 \\
\hline & & $m$ & 19 & 112 & 32 & 196 & -179 & 47 & -243 & 0.68 & 0.35 & 103 & 25 \\
\hline & & $\mathrm{m} / \mathrm{p}$ & 7 & 119 & 60 & 232 & -243 & 133 & -496 & 0.5 & 0.07 & 112 & 1 \\
\hline & & $p$ & 15 & 156 & 89 & 423 & -278 & 155 & -735 & 0.57 & 0.07 & 111 & 2 \\
\hline & All right & & 48 & 126 & 61 & 423 & -221 & 112 & -735 & 0.6 & 0.23 & 109 & 16 \\
\hline & All bites & & 78 & 173 & 105 & 532 & -345 & 243 & -1131 & 0.55 & 0.19 & 111 & 13 \\
\hline
\end{tabular}

a, anterior; a/m, anterior/middle; $\mathrm{m}$, middle; m/p, middle/posterior; p. posterior.

Principal strain orientations are axial circular data in which an $\varepsilon_{1}$ orientation of $0^{\circ}$ is equal to $180^{\circ}$ and the zero point is arbitrary. These data cannot be analyzed using traditional statistical methods. Quantitative analyses of in vivo principal strain data were performed in ORIANA 3.13 (Kovach Computing Services, Anglesey, UK; www.kovcomp.com). To conduct these analyses, all angle data were converted to positive values, thus $-30^{\circ}$ was converted to $330^{\circ}$. Additionally, ORIANA converts all axial data to values between 0 and $180^{\circ}$. Readers are urged to note these changes when comparing descriptive statistics from Tables $2-7$ to circular statistics from Tables 8-10.

Descriptive circular statistics (Tables 8-10) were calculated for $\varepsilon_{1}$ orientations at each gage site, with data grouped according to broad regions of the mandible (i.e. lateral, medial or ventral surfaces) and whether bites were ipsilateral or contralateral to the gage site (yielding working- and balancing-side bites). The statistics presented here include: the mean angle of the vectors $(\mu)$ relative to the reference axis describe above; length of the mean vector $(r)$, ranging from 0 to 1 , which is a measure of angular dispersion with values closer to 1 indicating that individual observations are clustered more closely around the mean; the concentration ( $k$, which measures the departure of the distribution from a uniform distribution (or perfect circle); circular variance (V), which is calculated as $\mathrm{V}=1-r$ and is equivalent to its linear counterpart; the circular standard deviation $(S)$, calculated as $S=[-2 \ln (2)]^{1 / 2}$; the standard error of the mean; and the 95 and $99 \%$ confidence intervals derived from standard error. Additionally, Rayleigh's test of uniformity and Watson's $U^{2}$ test were used to determine whether data are derived from a von Mises distribution (continuous probability distribution on a circle, not to be confused with von Mises stress). A more detailed review of circular statistics can be found in Zar (1999). 
Table 4 Descriptive statistics for principal $\left(\varepsilon_{1}\right.$ and $\left.\varepsilon_{2}\right)$ and $\left|\left(\varepsilon_{1} / \varepsilon_{2}\right)\right|$ strain magnitude and $\varepsilon_{1}$ orientation for experiment 99 .

\begin{tabular}{|c|c|c|c|c|c|c|c|c|c|c|c|c|c|}
\hline \multirow{2}{*}{$\begin{array}{l}\text { Gauge } \\
\text { location }\end{array}$} & \multirow[b]{2}{*}{ Bite side } & \multirow{2}{*}{$\begin{array}{l}\text { Bite } \\
\text { position }\end{array}$} & \multirow[b]{2}{*}{$n$} & \multicolumn{3}{|l|}{$\varepsilon_{1}$} & \multicolumn{3}{|l|}{$\varepsilon_{2}$} & \multicolumn{2}{|c|}{$\varepsilon_{1} / \varepsilon_{2}$ Ratio } & \multicolumn{2}{|c|}{$\begin{array}{l}\varepsilon_{1} \\
\text { Orientation }\end{array}$} \\
\hline & & & & Mean & SD & Max & Mean & SD & Max & Mean & SD & Mean & SD \\
\hline \multirow{10}{*}{$\begin{array}{l}\text { Right } \\
\text { dorsolateral } \\
\text { dentary }\end{array}$} & Midline & & 6 & 188 & 83 & 342 & -149 & 73 & -284 & 1.31 & 0.39 & -2 & 7 \\
\hline & Left (BS) & $\mathrm{m}$ & 2 & 272 & 3 & 274 & -201 & 1 & -202 & 1.35 & 0.01 & 20 & 0 \\
\hline & & $p$ & 1 & 137 & & 137 & -157 & & -157 & 0.87 & & -26 & \\
\hline & All left & & 3 & 227 & 78 & 342 & -186 & -26 & -202 & 1.19 & 0.27 & 4 & 27 \\
\hline & Right (WS) & $\mathrm{a} / \mathrm{m}$ & 2 & 797 & 583 & 1210 & -618 & 638 & -1069 & 1.72 & 0.82 & -25 & 21 \\
\hline & & $\mathrm{m}$ & 7 & 365 & 375 & 1188 & -145 & 185 & -554 & 3.1 & 0.86 & -18 & 14 \\
\hline & & $\mathrm{m} / \mathrm{p}$ & 2 & 52 & 9 & 58 & -2 & 1 & -2 & 54.33 & 20.53 & -22 & 1 \\
\hline & & $p$ & 9 & 382 & 399 & 1415 & -199 & 212 & -722 & 2.26 & 0.8 & -32 & 29 \\
\hline & All right & & 20 & 384 & 399 & 1415 & -202 & 274 & -1069 & 7.71 & 19.73 & -25 & 22 \\
\hline & All bites & & 29 & 327 & 342 & 1415 & -190 & 229 & -1069 & 5071 & 16.54 & -18 & 23 \\
\hline \multirow{12}{*}{$\begin{array}{l}\text { Right } \\
\text { lateral } \\
\text { dentary }\end{array}$} & Midline & & 6 & 608 & 185 & 966 & -589 & 157 & -892 & 1.02 & 0.05 & 16 & 25 \\
\hline & Left (BS) & a & 1 & 1072 & & 1072 & -894 & & -894 & 1.2 & & 31 & \\
\hline & & $\mathrm{m}$ & 3 & 1364 & 288 & 1630 & -1106 & 209 & -1299 & 1.23 & 0.03 & 30 & 0 \\
\hline & & $\mathrm{m} / \mathrm{p}$ & 4 & 572 & 70 & 676 & -444 & 60 & -531 & 1.29 & 0.02 & 29 & 1 \\
\hline & & $p$ & 2 & 756 & 172 & 877 & -594 & 145 & -697 & 1.27 & 0.02 & 27 & 2 \\
\hline & All left & & 10 & 897 & 388 & 1072 & -718 & 322 & -1299 & 1.26 & 0.04 & 29 & 2 \\
\hline & Right (WS) & $a / m$ & 2 & 1381 & 60 & 1424 & -1531 & 30 & -1553 & 0.9 & 0.02 & -47 & 1 \\
\hline & & $\mathrm{m}$ & 7 & 1229 & 578 & 2226 & -1192 & 490 & -1779 & 1.02 & 0.18 & -45 & 3 \\
\hline & & $\mathrm{m} / \mathrm{p}$ & 2 & 409 & 110 & 487 & -250 & 116 & -332 & 1.72 & 0.36 & -45 & 0 \\
\hline & & $p$ & 9 & 944 & 316 & 1436 & -606 & 199 & -986 & 1.63 & 0.63 & -50 & 6 \\
\hline & All right & & 20 & 1034 & 469 & 2226 & -868 & 501 & -1779 & 1.35 & 0.54 & -48 & 5 \\
\hline & All bites & & 36 & 925 & 433 & 2226 & -780 & 422 & -1779 & 1.27 & 0.42 & -6 & 55 \\
\hline \multirow{12}{*}{$\begin{array}{l}\text { Right } \\
\text { ventral } \\
\text { dentary }\end{array}$} & Midline & & 6 & 1072 & 218 & 1423 & -1938 & 385 & -2463 & 0.55 & 0.03 & 97 & 6 \\
\hline & Left (BS) & a & 1 & 1046 & & 1046 & -1712 & & -1712 & 0.61 & & 90 & \\
\hline & & $\mathrm{m}$ & 3 & 1313 & 277 & 1560 & -2132 & 459 & -2537 & 0.62 & 0 & 90 & 0 \\
\hline & & $\mathrm{m} / \mathrm{p}$ & 4 & 509 & 66 & 604 & -813 & 118 & -985 & 0.63 & 0.01 & 90 & 1 \\
\hline & & p & 2 & 832 & 462 & 1158 & -1453 & 849 & -2053 & 0.58 & 0.02 & 93 & 4 \\
\hline & All left & & 10 & 869 & 411 & 1560 & -1427 & 689 & -2537 & 0.61 & 0.02 & 91 & 2 \\
\hline & Right (WS) & $\mathrm{a} / \mathrm{m}$ & 2 & 960 & 180 & 1087 & -1711 & 626 & -2153 & 0.58 & 0.11 & -50 & 7 \\
\hline & & $\mathrm{m}$ & 7 & 754 & 295 & 1251 & -807 & 320 & -1230 & 1 & 0.35 & -32 & 16 \\
\hline & & $\mathrm{m} / \mathrm{p}$ & 2 & 428 & 82 & 486 & -187 & 43 & -217 & 2.31 & 0.09 & -2 & 1 \\
\hline & & $\mathrm{p}$ & 9 & 1922 & 685 & 2987 & -971 & 336 & -1470 & 1.97 & 0.1 & 4 & 2 \\
\hline & All right & & 20 & 1268 & 782 & 2987 & -909 & 478 & -2153 & 1.53 & 0.63 & -15 & 23 \\
\hline & All bites & & 36 & 1124 & 643 & 2987 & -1224 & 650 & -2537 & 1.11 & 0.66 & 33 & 57 \\
\hline \multirow{12}{*}{$\begin{array}{l}\text { Right } \\
\text { medial } \\
\text { splenial }\end{array}$} & Midline & & 6 & 765 & 131 & 967 & -1398 & 280 & -1847 & 0.55 & 0.02 & 53 & 6 \\
\hline & Left (BS) & a & 1 & 754 & & 754 & -1567 & & -1567 & 0.48 & & -43 & \\
\hline & & $\mathrm{m}$ & 3 & 920 & 142 & 1066 & -2139 & 512 & -2606 & 0.44 & 0.05 & -43 & 1 \\
\hline & & $\mathrm{m} / \mathrm{p}$ & 4 & 307 & 61 & 390 & -709 & 114 & -880 & 0.43 & 0.04 & -47 & 1 \\
\hline & & $\mathrm{p}$ & 2 & 632 & 261 & 816 & -1366 & 711 & -1868 & 0.48 & 0.06 & -46 & 3 \\
\hline & All left & & 10 & 601 & 297 & 1066 & -1355 & 717 & -2606 & 0.45 & 0.04 & -45 & 2 \\
\hline & Right (WS) & $a / m$ & 2 & 678 & 483 & 1019 & -1084 & 686 & -1569 & 0.61 & 0.06 & -80 & 2 \\
\hline & & $\mathrm{m}$ & 7 & 386 & 255 & 876 & -554 & 255 & -1032 & 0.66 & 0.13 & -70 & 19 \\
\hline & & $\mathrm{m} / \mathrm{p}$ & 2 & 183 & 36 & 209 & -272 & 33 & -295 & 0.67 & 0.05 & -37 & 1 \\
\hline & & $p$ & 9 & 518 & 468 & 1693 & -547 & 269 & -1181 & 0.84 & 0.28 & 35 & 32 \\
\hline & All right & & 20 & 455 & 376 & 1693 & -575 & 337 & -1569 & 0.74 & 0.22 & -38 & 57 \\
\hline & All bites & & 36 & 547 & 340 & 1693 & -929 & 605 & -2606 & 0.63 & 0.21 & -31 & 45 \\
\hline
\end{tabular}

a, anterior; $a / m$, anterior/middle; $m$, middle; $m / p$, middle/posterior; $p$, posterior.

To determine whether working and balancing sides strain regimes differ, principal strain orientations recorded within the same gage during ipsilateral vs. contralateral biting were compared using a nonparametric Mardia-Watson-Wheeler test (for data that did not match a von Mises distribution) or a parametric Watson-Williams F-test (when data matched a von Mises distribu- tion). These tests determine whether two or more distributions are identical; significant differences between distributions lead to a large $W$ statistic and low probability of distributions being identical. To determine whether strain orientation changed as load magnitude increased, circular-linear correlation coefficients were calculated between $\varepsilon_{1}$ orientation and magnitude (Zar, 1999). 
202 Alligator mandibular bone strain, L. B. Porro et al.

Table 5 Descriptive statistics for principal $\left(\varepsilon_{1}\right.$ and $\left.\varepsilon_{2}\right)$ and $\left|\left(\varepsilon_{1} / \varepsilon_{2}\right)\right|$ strain magnitude and $\varepsilon_{1}$ orientation for experiment 102.

\begin{tabular}{|c|c|c|c|c|c|c|c|c|c|c|c|c|c|}
\hline \multirow{2}{*}{$\begin{array}{l}\text { Gauge } \\
\text { location }\end{array}$} & \multirow[b]{2}{*}{ Bite side } & \multirow{2}{*}{$\begin{array}{l}\text { Bite } \\
\text { position }\end{array}$} & \multirow[b]{2}{*}{$n$} & \multicolumn{3}{|c|}{$\varepsilon_{1}$} & \multicolumn{3}{|c|}{$\varepsilon_{2}$} & \multicolumn{2}{|c|}{$\varepsilon_{1} / \varepsilon_{2}$ Ratio } & \multicolumn{2}{|c|}{$\begin{array}{c}\varepsilon_{1} \\
\text { Orientation }\end{array}$} \\
\hline & & & & Mean & SD & Max & Mean & SD & $\operatorname{Max}$ & Mean & SD & Mean & SD \\
\hline \multirow{10}{*}{$\begin{array}{l}\text { Left } \\
\text { dorsolateral } \\
\text { dentary }\end{array}$} & Left (WS) & $\mathrm{a} / \mathrm{m}$ & 3 & 916 & 470 & 1450 & -560 & 352 & -960 & 1.71 & 0.19 & -19 & 5 \\
\hline & & $\mathrm{m}$ & 12 & 1311 & 590 & 2256 & -753 & 335 & -1202 & 1.76 & 0.14 & -23 & 6 \\
\hline & & $\mathrm{m} / \mathrm{p}$ & 2 & 1290 & 763 & 1830 & -596 & 383 & -867 & 2.21 & 0.14 & -26 & 4 \\
\hline & & $\mathrm{p}$ & 5 & 1264 & 570 & 2244 & -565 & 267 & -889 & 2.39 & 0.63 & -79 & 12 \\
\hline & All left & & 22 & 1245 & 558 & 2256 & -670 & 316 & -1202 & 1.94 & 0.42 & -35 & 25 \\
\hline & Right (BS) & $\mathrm{m}$ & 5 & 1056 & 254 & 1414 & -1127 & 263 & -1472 & 0.94 & 0.03 & 27 & 2 \\
\hline & & $\mathrm{m} / \mathrm{p}$ & 4 & 1158 & 413 & 1642 & -1203 & 419 & -1678 & 0.96 & 0.04 & 24 & 2 \\
\hline & & $\mathrm{p}$ & 5 & 1372 & 539 & 1777 & -1379 & 581 & -1742 & 1.03 & 0.1 & 23 & 2 \\
\hline & All right & & 14 & 1198 & 410 & 1777 & -1239 & 422 & -1742 & 0.98 & 0.07 & 24 & 2 \\
\hline & All bites & & 36 & 1227 & 500 & 2256 & -891 & 453 & -1742 & 1.56 & 0.58 & -10 & 35 \\
\hline \multirow{14}{*}{$\begin{array}{l}\text { Left } \\
\text { lateral } \\
\text { dentary }\end{array}$} & Midline & & 3 & 844 & 183 & 985 & -973 & 151 & -1078 & 0.86 & 0.06 & -39 & 1 \\
\hline & Left (WS) & a & 2 & 337 & 70 & 426 & -509 & 133 & -603 & 0.75 & 0.06 & -35 & 2 \\
\hline & & $\mathrm{a} / \mathrm{m}$ & 9 & 860 & 246 & 1207 & -876 & 293 & -1338 & 1.01 & 0.15 & -39 & 1 \\
\hline & & $\mathrm{m}$ & 19 & 1482 & 696 & 2728 & -1301 & 636 & -2142 & 1.16 & 0.13 & -38 & 9 \\
\hline & & $\mathrm{m} / \mathrm{p}$ & 6 & 1231 & 728 & 2589 & -829 & 645 & -1801 & 2.15 & 1.83 & -38 & 4 \\
\hline & & $p$ & 11 & 1174 & 488 & 2312 & -183 & 198 & -629 & 11.08 & 8.68 & -47 & 18 \\
\hline & All left & & 47 & 1212 & 628 & 2728 & -864 & 650 & -2142 & 3.4 & 5.69 & -40 & 11 \\
\hline & Right (BS) & a & 2 & 836 & 48 & 870 & -851 & 24 & -868 & 0.98 & 0.03 & 40 & 0 \\
\hline & & $\mathrm{a} / \mathrm{m}$ & 2 & 1308 & 159 & 1420 & -1343 & 202 & -1486 & 0.98 & 0.03 & 40 & 1 \\
\hline & & $\mathrm{m}$ & 8 & 1457 & 223 & 1694 & -1525 & 248 & -1800 & 0.96 & 0.01 & 37 & 1 \\
\hline & & $\mathrm{m} / \mathrm{p}$ & 11 & 1232 & 389 & 1855 & -1279 & 449 & -2027 & 0.98 & 0.07 & 36 & 1 \\
\hline & & $\mathrm{p}$ & 13 & 1336 & 465 & 1901 & -1413 & 541 & -2072 & 0.97 & 0.08 & 36 & 2 \\
\hline & All right & & 36 & 1302 & 385 & 1901 & -1362 & 442 & -2072 & 0.97 & 0.06 & 36 & 2 \\
\hline & All bites & & 83 & 1227 & 532 & 2728 & -1071 & 608 & -2142 & 2.25 & 4.34 & -4 & 39 \\
\hline \multirow{14}{*}{$\begin{array}{l}\text { Left } \\
\text { ventral } \\
\text { dentary }\end{array}$} & Midline & & 3 & 590 & 24 & 618 & -1066 & 105 & -1142 & 0.56 & 0.05 & 60 & 1 \\
\hline & Left (WS) & a & 2 & 390 & 45 & 422 & -383 & 69 & -432 & 1.03 & 0.07 & -39 & 1 \\
\hline & & $\mathrm{a} / \mathrm{m}$ & 9 & 296 & 68 & 368 & -316 & 60 & -454 & 0.96 & 0.24 & -39 & 12 \\
\hline & & $\mathrm{m}$ & 19 & 291 & 95 & 413 & -420 & 136 & -787 & 0.72 & 0.23 & -37 & 13 \\
\hline & & $\mathrm{m} / \mathrm{p}$ & 6 & 188 & 53 & 282 & -377 & 119 & -558 & 0.56 & 0.28 & -57 & 22 \\
\hline & & $\mathrm{p}$ & 11 & 540 & 391 & 1641 & -569 & 375 & -1266 & 1.11 & 0.56 & -81 & 42 \\
\hline & All left & & 47 & 341 & 228 & 1641 & -428 & 219 & -1266 & 0.85 & 0.38 & -50 & 29 \\
\hline & Right (BS) & a & 2 & 330 & 101 & 401 & -747 & 98 & -816 & 0.44 & 0.08 & -62 & 2 \\
\hline & & $\mathrm{a} / \mathrm{m}$ & 2 & 511 & 174 & 633 & -1127 & 371 & -1390 & 0.45 & 0.01 & -63 & 0 \\
\hline & & $\mathrm{m}$ & 8 & 659 & 113 & 829 & -1488 & 281 & -1935 & 0.44 & 0.02 & -68 & 3 \\
\hline & & $\mathrm{m} / \mathrm{p}$ & 11 & 547 & 210 & 924 & -1344 & 545 & -2326 & 0.41 & 0.03 & -65 & 3 \\
\hline & & $\mathrm{p}$ & 12 & 598 & 248 & 989 & -1470 & 648 & -2527 & 0.41 & 0.03 & -66 & 3 \\
\hline & All right & & 35 & 576 & 206 & 989 & -1374 & 526 & -2527 & 0.42 & 0.03 & -66 & 3 \\
\hline & All bites & & 82 & 445 & 244 & 1641 & -839 & 595 & -2527 & 0.66 & 0.35 & -47 & 23 \\
\hline \multirow{14}{*}{$\begin{array}{l}\text { Left } \\
\text { ventral } \\
\text { splenial }\end{array}$} & Midline & & 1 & 723 & & 723 & -1900 & & -1900 & 0.38 & & 89 & \\
\hline & Left (WS) & $a$ & 2 & 638 & 237 & 805 & -1527 & 531 & -1902 & 0.42 & 0.01 & -82 & 23 \\
\hline & & $\mathrm{a} / \mathrm{m}$ & 6 & 455 & 141 & 615 & -966 & 301 & -1366 & 0.47 & 0.02 & -80 & 2 \\
\hline & & $\mathrm{m}$ & 7 & 258 & 128 & 407 & -365 & 141 & -519 & 0.69 & 0.2 & -70 & 15 \\
\hline & & $\mathrm{m} / \mathrm{p}$ & 4 & 375 & 211 & 664 & -356 & 232 & -668 & 1.31 & 0.79 & -33 & 44 \\
\hline & & $\mathrm{p}$ & 4 & 1482 & 481 & 1794 & -768 & 149 & -896 & 1.89 & 0.42 & 11 & 4 \\
\hline & All left & & 23 & 576 & 492 & 1794 & -691 & 435 & -1902 & 0.93 & 0.64 & -53 & 39 \\
\hline & Right (BS) & $a$ & 2 & 341 & 159 & 454 & -994 & 330 & -1228 & 0.33 & 0.05 & -98 & 4 \\
\hline & & $\mathrm{a} / \mathrm{m}$ & 2 & 554 & 319 & 780 & -1709 & 742 & -2234 & 0.31 & 0.05 & -95 & 4 \\
\hline & & $\mathrm{m}$ & 3 & 781 & 112 & 907 & -2300 & 357 & -2670 & 0.34 & 0.01 & -92 & 1 \\
\hline & & $\mathrm{m} / \mathrm{p}$ & 7 & 481 & 251 & 812 & -1374 & 778 & -2390 & 0.4 & 0.19 & -105 & 35 \\
\hline & & $p$ & 8 & 662 & 306 & 1187 & -1773 & 881 & -3454 & 0.38 & 0.02 & -93 & 1 \\
\hline & All right & & 22 & 581 & 271 & 1187 & -1641 & 780 & -3454 & 0.37 & 0.11 & -97 & 19 \\
\hline & All bites & & 46 & 576 & 391 & 1794 & -1174 & 783 & -3454 & 0.64 & 0.54 & 58 & 37 \\
\hline
\end{tabular}


Table 5. (continued)

\begin{tabular}{|c|c|c|c|c|c|c|c|c|c|c|c|c|c|}
\hline \multirow{2}{*}{$\begin{array}{l}\text { Gauge } \\
\text { location }\end{array}$} & \multirow[b]{2}{*}{ Bite side } & \multirow{2}{*}{$\begin{array}{l}\text { Bite } \\
\text { position }\end{array}$} & \multirow[b]{2}{*}{$n$} & \multicolumn{3}{|c|}{$\varepsilon_{1}$} & \multicolumn{3}{|c|}{$\varepsilon_{2}$} & \multicolumn{2}{|c|}{$\varepsilon_{1} / \varepsilon_{2}$ Ratio } & \multicolumn{2}{|c|}{$\begin{array}{c}\varepsilon_{1} \\
\text { Orientation }\end{array}$} \\
\hline & & & & Mean & SD & Max & Mean & SD & Max & Mean & SD & Mean & SD \\
\hline \multirow{14}{*}{$\begin{array}{l}\text { Left } \\
\text { medial } \\
\text { splenial }\end{array}$} & Midline & & 3 & 582 & 118 & 698 & -839 & 95 & -948 & 0.69 & 0.09 & 148 & 7 \\
\hline & Left (WS) & $\mathrm{a}$ & 2 & 449 & 160 & 562 & -959 & 338 & -1198 & 0.47 & 0 & 113 & 1 \\
\hline & & $\mathrm{a} / \mathrm{m}$ & 9 & 512 & 175 & 773 & -903 & 363 & -1428 & 0.59 & 0.09 & 100 & 6 \\
\hline & & $\mathrm{m}$ & 19 & 654 & 319 & 1070 & -927 & 451 & -1549 & 0.71 & 0.1 & 93 & 9 \\
\hline & & $\mathrm{m} / \mathrm{p}$ & 6 & 577 & 250 & 950 & -560 & 382 & -1177 & 1.33 & 0.78 & 72 & 27 \\
\hline & & $\mathrm{p}$ & 11 & 956 & 252 & 1401 & -363 & 144 & -608 & 2.86 & 0.79 & 40 & 9 \\
\hline & All left & & 47 & 679 & 306 & 1401 & -745 & 431 & -1549 & 1.26 & 1.03 & 80 & 27 \\
\hline & Right (BS) & a & 2 & 514 & 39 & 541 & -548 & 27 & -567 & 0.94 & 0.12 & -15 & 5 \\
\hline & & $\mathrm{a} / \mathrm{m}$ & 2 & 764 & 49 & 798 & -837 & 145 & -939 & 0.92 & 0.1 & -17 & 4 \\
\hline & & $\mathrm{m}$ & 8 & 810 & 109 & 905 & -903 & 131 & -1027 & 0.9 & 0.03 & -15 & 1 \\
\hline & & $\mathrm{m} / \mathrm{p}$ & 11 & 666 & 192 & 966 & -757 & 267 & -1218 & 0.9 & 0.08 & -15 & 3 \\
\hline & & $\mathrm{p}$ & 12 & 733 & 231 & 1010 & -825 & 329 & -1246 & 0.97 & 0.19 & -14 & 7 \\
\hline & All right & & 35 & 71 & 190 & 1010 & -806 & 260 & -1246 & 0.93 & 0.14 & -15 & 5 \\
\hline & All bites & & 82 & 688 & 259 & 1401 & -770 & 360 & -1549 & 1.1 & 0.78 & 41 & 53 \\
\hline
\end{tabular}

a, anterior; $a / m$, anterior/middle; $\mathrm{m}$, middle; $\mathrm{m} / \mathrm{p}$, middle/posterior; $\mathrm{p}$, posterior.

Lastly, two-way ANOvas were carried out using the Circular Statistics Toolbox for MATLAB (Berens, 2009) to determine whether differences in $\varepsilon_{1}$ orientations from gage sites located in dorsolateral, lateral, ventral and medial areas of the jaw across all experiments were due to changes in bite point or individual variation (Table 11).

All statistical tests discussed below were conducted using sPSS v11.5 (SPSS Inc., Chicago, IL). Shear strain data were averaged across in vivo experiments (and not sorted into different bite point locations for this analysis); summarized results for the entire mandible and for the dentary and splenial bones are indicated in Table 12. To quantitatively evaluate the degree of variation in strain orientation with changes in bite point, ANOVAS were performed to assess whether significant differences in $\varepsilon_{1}$ orientation existed among bite points for individual gages during all experiments (Table 13).

\section{Results}

\section{Principal strain $\left(\varepsilon_{1}\right)$ orientations: lateral mandible}

In vivo strain orientations

During biting ipsilateral to the gages (i.e. in the working side mandible) $\varepsilon_{1}$ strains on the lateral aspect of the mandible (dorsolateral and lateral gages) are oriented anteroinferiorly, between -12 and $-79^{\circ}$ from the reference axis (Fig. 2, Tables 3-6). The only exception is the lateral gage in Experiment 103, which exhibits $\varepsilon_{1}$ strains oriented anterosuperiorly at an angle of approximately $30^{\circ}$. With the exception of Experiment 75, $\varepsilon_{1}$ strain orientations tend to rotate clockwise as the bite point moves posteriorly.

During biting contralateral to the gage site (i.e. in the balancing side mandible) (Fig. 3, Tables 3-6), $\varepsilon_{1}$ is oriented anterosuperiorly between 23 and $40^{\circ}$ from the reference axis. Exceptions include the dorsolateral gage in Experiment $99\left(\varepsilon_{1}\right.$ orientations ranging from 20 to $\left.-26^{\circ}\right)$ and, as during ipsilateral biting, the lateral gage in Experiment 103 in which $\varepsilon_{1}$ is oriented posterosuperiorly. Contralateral biting does not produce any observable trends of changes in $\varepsilon_{1}$ orientation with changes in bite point. Mean vector length and concentration (Table 8 ) reveal that $\varepsilon_{1}$ strains are more concentrated (i.e. orientations are less variable) during contralateral than ipsilateral biting.

In all experiments, Mardia-Watson-Wheeler and Wheeler-Williams tests show that lateral gage $\varepsilon_{1}$ orientations during ipsilateral bites are significantly different from those recorded during contralateral biting (Table 8). Twoway ANOVA (Table 11) suggests that bite point has a significant impact on $\varepsilon_{1}$ orientations in lateral gages (ipsilateral and contralateral biting) and dorsolateral gages (ipsilateral biting) but not in dorsolateral gages during contralateral bites. Additionally, analyses reveal significant differences in $\varepsilon_{1}$ orientation between similarly located gages (dorsolateral and lateral gages) in different experiments when both biting side and bite point are taken into account (Table 11); this may be due to slight differences in gage location between experiments or individual differences between research subjects. For most dorsolateral and lateral gage sites, strain orientation appears to be strongly correlated with strain magnitude (Table 8), although these comparisons are rarely statistically significant.

\section{FEM strain orientations}

Working side strain orientations at dorsolateral gages are directed horizontally to anteroinferiorly, ranging from +1 to $-83^{\circ}$ (Fig. 2, Table 7). At working side lateral gage locations, orientations range from superior $\left(90^{\circ}\right)$ to anteroinferior $\left(-78^{\circ}\right) . \varepsilon_{1}$ orientations tend to rotate clockwise 
Table 6 Descriptive statistics for principal $\left(\varepsilon_{1}\right.$ and $\left.\varepsilon_{2}\right)$ and $\left|\left(\varepsilon_{1} / \varepsilon_{2}\right)\right|$ strain magnitude and $\varepsilon_{1}$ orientation for experiment 103.

\begin{tabular}{|c|c|c|c|c|c|c|c|c|c|c|c|c|c|}
\hline \multirow{2}{*}{$\begin{array}{l}\text { Gauge } \\
\text { location }\end{array}$} & \multirow[b]{2}{*}{ Bite side } & \multirow{2}{*}{$\begin{array}{l}\text { Bite } \\
\text { position }\end{array}$} & \multirow[b]{2}{*}{$n$} & \multicolumn{3}{|c|}{$\varepsilon_{1}$} & \multicolumn{3}{|c|}{$\varepsilon_{2}$} & \multicolumn{2}{|c|}{$\varepsilon_{1} / \varepsilon_{2}$ Ratio } & \multicolumn{2}{|c|}{$\begin{array}{c}\varepsilon_{1} \\
\text { Orientation }\end{array}$} \\
\hline & & & & Mean & SD & Max & Mean & SD & Max & Mean & SD & Mean & SD \\
\hline \multirow{11}{*}{$\begin{array}{l}\text { Right } \\
\text { dorsolateral } \\
\text { dentary }\end{array}$} & \multirow[t]{4}{*}{ Left (BS) } & $\mathrm{a} / \mathrm{m}$ & 6 & 1334 & 282 & 1751 & -1141 & 246 & -1489 & 1.17 & 0.03 & 38 & 2 \\
\hline & & $\mathrm{m}$ & 3 & 1380 & 248 & 1606 & -1176 & 179 & -1335 & 1.17 & 0.03 & 37 & 1 \\
\hline & & $\mathrm{m} / \mathrm{p}$ & 6 & 859 & 435 & 1430 & -655 & 372 & -1115 & 1.38 & 0.17 & 37 & 3 \\
\hline & & $\mathrm{p}$ & 4 & 1021 & 188 & 1301 & -817 & 147 & -1021 & 1.25 & 0.06 & 37 & 2 \\
\hline & All left & & 19 & 1125 & 373 & 1751 & -925 & 340 & -1489 & 1.25 & 0.13 & 37 & 3 \\
\hline & \multirow[t]{4}{*}{ Right (WS) } & $\mathrm{a} / \mathrm{m}$ & 3 & 1411 & 81 & 1503 & -1122 & 148 & -1254 & 1.27 & 0.12 & -12 & 5 \\
\hline & & $\mathrm{m}$ & 1 & 874 & & 874 & -593 & & -593 & 1.47 & & -14 & \\
\hline & & $\mathrm{m} / \mathrm{p}$ & 4 & 1257 & 570 & 1825 & -113 & 651 & -170 & 24.99 & 34.79 & -58 & 22 \\
\hline & & $p$ & 3 & 2506 & 128 & 2651 & -285 & 8 & -292 & 8.79 & 0.7 & -78 & 2 \\
\hline & All right & & 11 & 1605 & 677 & 2651 & -479 & 442 & -1254 & 11.96 & 21.89 & -47 & 31 \\
\hline & All bites & & 30 & 1301 & 547 & 2651 & -761 & 432 & -1489 & 5.18 & 13.89 & 6 & 45 \\
\hline \multirow{11}{*}{$\begin{array}{l}\text { Right } \\
\text { lateral } \\
\text { dentary }\end{array}$} & \multirow[t]{4}{*}{ Left (BS) } & $\mathrm{a} / \mathrm{m}$ & 6 & 1385 & 249 & 1775 & -1588 & 328 & -2039 & 0.88 & 0.06 & 115 & 1 \\
\hline & & $\mathrm{m}$ & 9 & 1597 & 231 & 1858 & -1821 & 260 & -2129 & 0.88 & 0.01 & 114 & 1 \\
\hline & & $\mathrm{m} / \mathrm{p}$ & 6 & 823 & 364 & 1248 & -827 & 484 & -1426 & 1.11 & 0.28 & 108 & 7 \\
\hline & & $p$ & 9 & 1155 & 441 & 1911 & -1267 & 530 & -2170 & 0.92 & 0.06 & 113 & 2 \\
\hline & All left & & 30 & 1267 & 428 & 1911 & -1409 & 539 & -2170 & 0.94 & 0.15 & 113 & 4 \\
\hline & \multirow[t]{4}{*}{ Right (WS) } & $\mathrm{a} / \mathrm{m}$ & 9 & 1720 & 604 & 2958 & -1489 & 157 & -1727 & 1.15 & 0.38 & 31 & 2 \\
\hline & & $\mathrm{m}$ & 3 & 2018 & 842 & 2835 & -840 & 731 & -1511 & 16.29 & 25.98 & 35 & 5 \\
\hline & & $\mathrm{m} / \mathrm{p}$ & 4 & 1870 & 670 & 2709 & -328 & 258 & -648 & 10.53 & 8.83 & 31 & 5 \\
\hline & & $p$ & 8 & 2088 & 281 & 2417 & -112 & 75 & -265 & 49.39 & 84.26 & 32 & 4 \\
\hline & All right & & 24 & 1905 & 546 & 2958 & -755 & 673 & -1727 & 20.69 & 51.83 & 32 & 4 \\
\hline & All bites & & 54 & 1551 & 576 & 2958 & -1119 & 681 & -2170 & 9.72 & 35.55 & 77 & 50 \\
\hline \multirow{11}{*}{$\begin{array}{l}\text { Right } \\
\text { ventral } \\
\text { dentary }\end{array}$} & \multirow[t]{4}{*}{ Left (BS) } & $\mathrm{a} / \mathrm{m}$ & 6 & 846 & 164 & 1087 & -822 & 181 & -1045 & 1.04 & 0.07 & 103 & 3 \\
\hline & & $\mathrm{m}$ & 9 & 882 & 91 & 963 & -799 & 67 & -896 & 1.1 & 0.07 & 103 & 0 \\
\hline & & $\mathrm{m} / \mathrm{p}$ & 6 & 486 & 255 & 790 & -470 & 282 & -821 & 1.1 & 0.14 & 97 & 9 \\
\hline & & $\mathrm{p}$ & 9 & 662 & 218 & 1041 & -617 & 187 & -946 & 1.07 & 0.04 & 103 & 2 \\
\hline & All left & & 30 & 730 & 234 & 1087 & -683 & 222 & -1045 & 1.08 & 0.08 & 102 & 5 \\
\hline & \multirow[t]{4}{*}{ Right (WS) } & $\mathrm{a} / \mathrm{m}$ & 9 & 657 & 150 & 843 & -1007 & 346 & -1398 & 0.69 & 0.15 & -21 & 14 \\
\hline & & $\mathrm{m}$ & 3 & 687 & 444 & 1193 & -655 & 146 & -816 & 0.99 & 0.42 & 3 & 31 \\
\hline & & $\mathrm{m} / \mathrm{p}$ & 4 & 623 & 266 & 880 & -317 & 174 & -499 & 2.33 & 1.28 & 26 & 9 \\
\hline & & $\mathrm{p}$ & 8 & 974 & 242 & 1331 & -572 & 151 & -829 & 1.72 & 0.26 & 36 & 2 \\
\hline & All right & & 24 & 761 & 276 & 1331 & -703 & 350 & -1398 & 1.35 & 0.81 & 7 & 29 \\
\hline & All bites & & 54 & 743 & 252 & 1331 & -692 & 283 & -1398 & 1.2 & 0.55 & 60 & 47 \\
\hline \multirow{11}{*}{$\begin{array}{l}\text { Right } \\
\text { medial } \\
\text { splenial }\end{array}$} & \multirow[t]{4}{*}{ Left (BS) } & $\mathrm{a} / \mathrm{m}$ & 6 & 861 & 187 & 1097 & -2108 & 532 & -2806 & 0.41 & 0.03 & 99 & 0 \\
\hline & & $\mathrm{m}$ & 7 & 1018 & 159 & 1176 & -2500 & 383 & -2828 & 0.41 & 0.03 & 100 & 1 \\
\hline & & $\mathrm{m} / \mathrm{p}$ & 6 & 662 & 275 & 1055 & -1326 & 792 & -2445 & 0.57 & 0.15 & 99 & 1 \\
\hline & & $\mathrm{p}$ & 8 & 788 & 334 & 1512 & -1735 & 757 & -3436 & 0.45 & 0.03 & 99 & 1 \\
\hline & All left & & 27 & 833 & 273 & 1512 & -1926 & 744 & -3436 & 0.46 & 0.1 & 99 & 1 \\
\hline & Right (WS) & $\mathrm{a} / \mathrm{m}$ & 7 & 651 & 397 & 1534 & -465 & 176 & -733 & 1.44 & 0.62 & 74 & 12 \\
\hline & & $\mathrm{m}$ & 1 & 432 & & 432 & -426 & & -426 & 0.01 & & 94 & \\
\hline & & $\mathrm{m} / \mathrm{p}$ & 4 & 724 & 476 & 1176 & -998 & 481 & -1321 & 0.68 & 0.21 & -23 & 78 \\
\hline & & $p$ & 3 & 2084 & 701 & 2565 & -1743 & 121 & -1878 & 1.22 & 0.47 & -7 & 2 \\
\hline & All right & & 15 & 942 & 735 & 2565 & -860 & 574 & -1878 & 1.16 & 0.56 & 35 & 49 \\
\hline & All bites & & 42 & 872 & 484 & 2565 & -1545 & 855 & -3436 & 0.71 & 0.48 & 67 & 43 \\
\hline
\end{tabular}

$a$, anterior; $a / m$, anterior/middle; $m$, middle; $m / p$, middle/posterior; $p$, posterior.

as bite point moves posteriorly, although there is no clear trend for FEM gage site 4, corresponding to the lateral gage of Experiment 75. Strain orientations on the lateral side of the mandible vary with gage location and bite point, as seen in FEM strain fields (strain fields are closeup views of the FEM with vectors illustrating $\varepsilon_{1}$ orientation for each element) (Fig. 4). During anterior and middle bites, $\varepsilon_{1}$ is oriented anteroinferiorly at all lateral gage sites; however, during posterior biting (when the bite point is immediately superior to the gages) there are strong local variations in strain orientation.

On the balancing side (Fig. 3, Table 7), $\varepsilon_{1}$ orientations are directed horizontally to anteroinferiorly for dorsolateral gages (ranging from 1 to $-55^{\circ}$ ) but anterosuperiorly (from 
Fig. 2 Orientations of maximum principal strain $\left(\varepsilon_{1}\right)$ on the working side mandible. Colored arrows represent variations in bite point in in vivo experiments (see inset for color key); strain orientation for every recorded bite during experiments is shown for every gage site in five experiments for lateral $(B, E, H, K)$, ventral $(A, C, F, I, L)$ and medial $(D, G, J, M)$ views. Black arrows represent strain orientations during anterior (solid line) and posterior bites (broken line) in the FEM (finite element model) in areas corresponding to in vivo gage sites. Note difference in scale between in vivo and FEM strain in the inset.

\section{A}

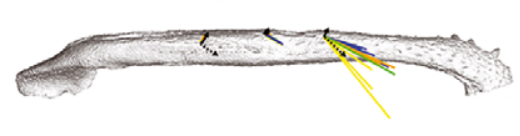

Experiment 56

B

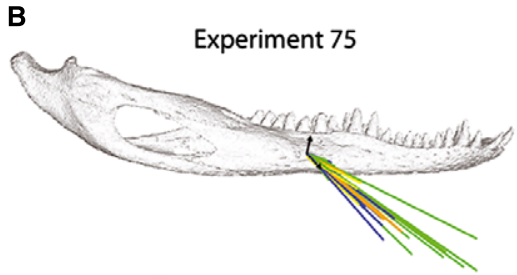

C
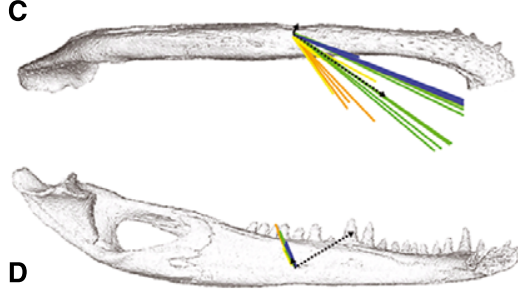

H Experiment 102

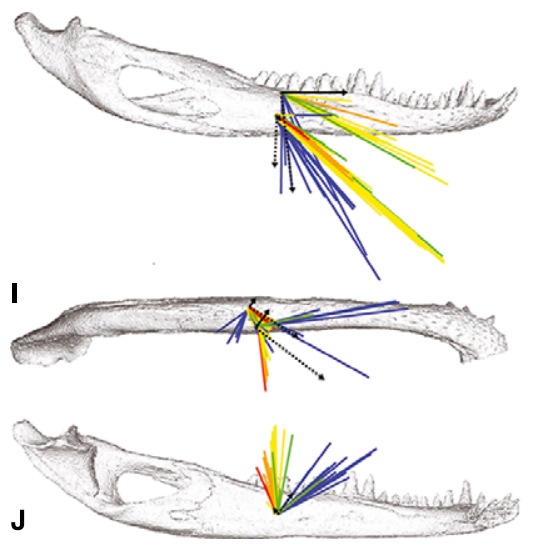

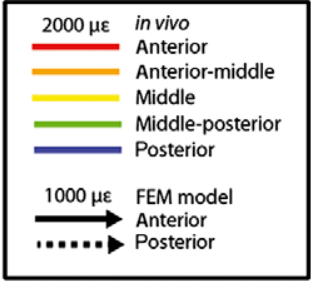

E Experiment 99

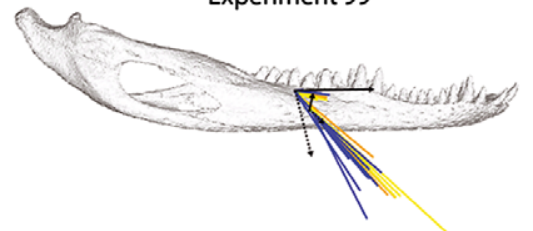

$\mathbf{F}$
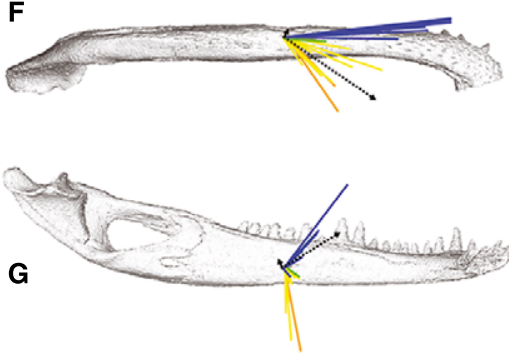

K

Experiment 103

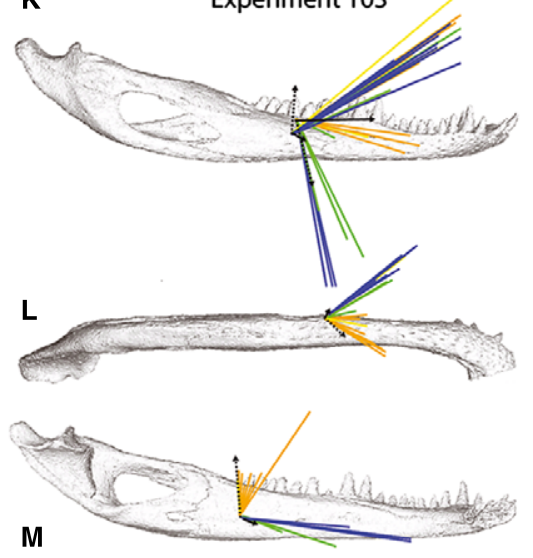

29 to $38^{\circ}$ ) for lateral gages. $\varepsilon_{1}$ orientations rotate clockwise as bite point moves posteriorly; however, $\varepsilon_{1}$ orientation does not vary by more than $5^{\circ}$ within any balancing side dorsolateral or lateral gage site. Strain fields (Fig. 4) reveal that the strain orientations on the lateral aspect of the balancing side remain similar across gage sites.

Strain orientations within dorsolateral and lateral gages vary less with changes in bite point on the balancing side (Fig. 3) than on the working side of the FEM (Figs 2 and 4). The range of strain orientations predicted by the FEM overlapped the range of in vivo $\varepsilon_{1}$ orientations recorded in dorsolateral gages (Experiments 99, 102, 103) during ipsilateral biting, lateral gages (Experiments 75, 99 , 102) during ipsilateral and contralateral biting, and the lateral gage of Experiment 103 during ipsilateral biting. In contrast, FEM strain orientations did not match in vivo orientations at dorsolateral gages during contralateral biting, and were nearly perpendicular to $\varepsilon_{1}$ orientations recorded at the lateral gage of Experiment 103 during contralateral biting; in this context, it should be observed that the strain orientations recorded during Experiment 103 are anomalous.

\section{Principal strain $\left(\varepsilon_{1}\right)$ orientations: medial mandible}

In vivo strain orientations

In vivo strain orientations recorded on the medial aspect of the mandible during ipsilateral biting (Fig. 2, Tables 3-6) are highly variable both within and between experiments, and it is difficult to make generalizations regarding the strain orientations for this region. In contrast, during contralateral biting, $\varepsilon_{1}$ on the medial aspect of the mandible is con- 


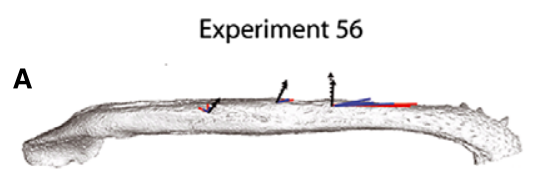

B
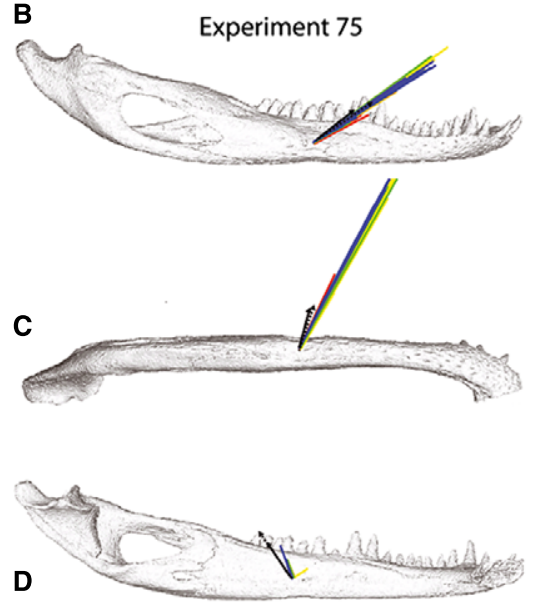

Experiment 102
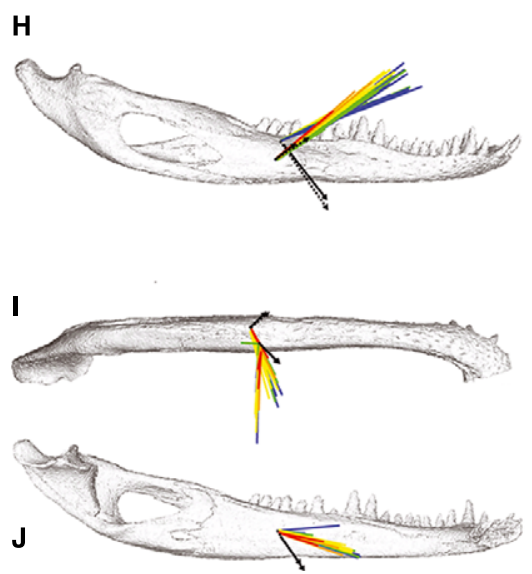

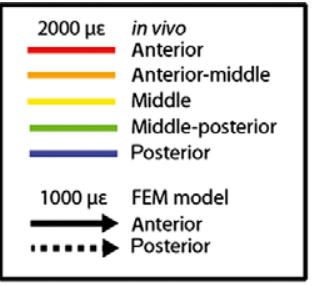

E
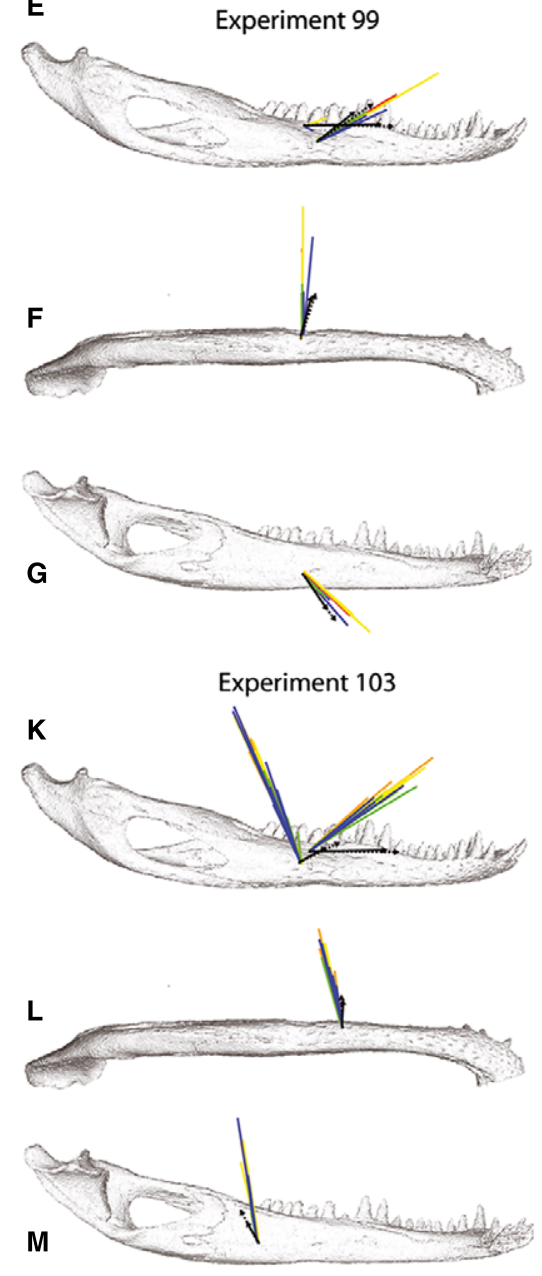

Fig. 3 Orientations of maximum principal strain $\left(\varepsilon_{1}\right)$ on the balancing side mandible. Colored arrows represent variations in bite point in in vivo experiments (see inset for color key); strain orientation for every recorded bite during experiments is shown for every gage site in five experiments for lateral $(B, E, H, K)$, ventral $(A, C, F, I, L)$ and medial $(D, G, J, M)$ views. Black arrows represent strain orientations during anterior (solid line) and posterior bites (broken line) in the FEM (finite element model) in areas corresponding to in vivo gage sites. Note difference in scale between in vivo and FEM strain in the inset. sistently oriented anteroinferiorly from the reference axis (Fig. 3; Tables 3-6) with values ranging from -14 to $-81^{\circ}$. Neither ipsilateral nor contralateral biting produce any observable trends of changes in $\varepsilon_{1}$ orientation with changes in bite point. As with lateral gages, $\varepsilon_{1}$ strains on the medial aspect of the mandible are more concentrated during contralateral biting than during ipsilateral biting (Table 9).

In all experiments, medial $\varepsilon_{1}$ orientations during ipsilateral bites are significantly different from those recorded during contralateral biting (Table 9). Two-way ANOvA (Table 11) suggests that differences in gage site/research subject across experiments has a significant impact on medial $\varepsilon_{1}$ orientations (ipsilateral and contralateral biting) and that bite point has a significant impact on medial $\varepsilon_{1}$ orien- tations during ipsilateral biting but not during contralateral biting. Circular-linear correlations do not reveal strong relationships between strain orientation and magnitude at most medial gage sites (Table 9).

\section{FEM strain orientations}

Strain orientations on the medial working side of the FEM were directed anterosuperiorly to anteroinferiorly, ranging from 32 to $-86^{\circ}$ (Fig. 2, Table 7). On the balancing side (Fig. 3, Table 7), $\varepsilon_{1}$ orientations were directed anteroinferiorly, ranging from -54 to $-63^{\circ}$, and varied by no more $4^{\circ}$ within any individual gage site, again demonstrating that strain orientations varied less with changes in bite point on the balancing side than on the working side of the FEM. 
Table 7 Descriptive statistics for principal $\left(\varepsilon_{1}\right.$ and $\left.\varepsilon_{2}\right)$ and $\left|\left(\varepsilon_{1} / \varepsilon_{2}\right)\right|$ strain magnitude and $\varepsilon_{1}$ orientation for the gage sites in the FEM. Maximum and mean values as well as standard deviations are based on results from bricks within gage sites.

\begin{tabular}{|c|c|c|c|c|c|c|c|c|c|c|c|c|c|}
\hline \multirow[b]{2}{*}{ Gage ID } & \multirow[b]{2}{*}{ Gage side } & \multirow{2}{*}{$\begin{array}{l}\text { No. of } \\
\text { bricks }\end{array}$} & \multirow{2}{*}{$\begin{array}{l}\text { Bite } \\
\text { position* }\end{array}$} & \multicolumn{3}{|c|}{ E1 } & \multicolumn{3}{|c|}{ E2 } & \multicolumn{2}{|c|}{$\begin{array}{c}\text { E1 } \\
\text { orientation }\end{array}$} & \multicolumn{2}{|c|}{ E1/E2 ratio } \\
\hline & & & & Mean & SD & Max & Mean & SD & Max & Mean & SD & Mean & SD \\
\hline \multirow{10}{*}{$\begin{array}{l}1 \text { Ventral } \\
\text { anterior } \\
\text { dentary }\end{array}$} & \multirow[t]{5}{*}{ Right (WS) } & \multirow[t]{5}{*}{24} & $\mathrm{a}$ & 61 & 8 & 71 & -291 & 28 & -342 & 64 & 2 & 0.21 & 0.02 \\
\hline & & & $\mathrm{a} / \mathrm{m}$ & 63 & 4 & 72 & -226 & 10 & -243 & 74 & 2 & 0.28 & 0.02 \\
\hline & & & $\mathrm{m}$ & 57 & 8 & 73 & -77 & 19 & -115 & -84 & 8 & 0.81 & 0.3 \\
\hline & & & $\mathrm{m} / \mathrm{p}$ & 136 & 44 & 213 & -37 & 16 & -61 & -51 & 9 & 5.21 & 6.19 \\
\hline & & & $\mathrm{p}$ & 176 & 41 & 248 & -37 & 14 & -59 & -43 & 3 & 6 & 6 \\
\hline & \multirow[t]{5}{*}{ Left (BS) } & \multirow[t]{5}{*}{25} & $a$ & 162 & 24 & 199 & -436 & 52 & -570 & 87 & 4 & 0.37 & 0.05 \\
\hline & & & $\mathrm{a} / \mathrm{m}$ & 187 & 27 & 228 & -451 & 52 & -587 & 90 & 4 & 0.42 & 0.05 \\
\hline & & & $\mathrm{m}$ & 199 & 29 & 242 & -456 & 51 & -591 & 91 & 4 & 0.44 & 0.06 \\
\hline & & & $\mathrm{m} / \mathrm{p}$ & 204 & 29 & 248 & -458 & 51 & -592 & 91 & 4 & 0.45 & 0.06 \\
\hline & & & $\mathrm{p}$ & 203 & 29 & 246 & -444 & 47 & -569 & 92 & 4 & 0.46 & 0.06 \\
\hline \multirow{10}{*}{$\begin{array}{l}2 \text { Ventral } \\
\text { posterior } \\
\text { dentary }\end{array}$} & \multirow[t]{5}{*}{ Right (WS) } & \multirow[t]{5}{*}{21} & a & 50 & 9 & 68 & -305 & 37 & -357 & 71 & 2 & 0.16 & 0.02 \\
\hline & & & $\mathrm{a} / \mathrm{m}$ & 54 & 8 & 74 & -380 & 31 & -327 & 77 & 2 & 0.19 & 0.02 \\
\hline & & & $\mathrm{m}$ & 44 & 5 & 54 & -160 & 13 & -192 & 80 & 2 & 0.28 & 0.04 \\
\hline & & & $\mathrm{m} / \mathrm{p}$ & 43 & 8 & 59 & -74 & 11 & -103 & 88 & 4 & 0.59 & 0.11 \\
\hline & & & $p$ & 0 & 0 & 0 & 2 & 0 & 2 & -5 & 7 & 0 & 0 \\
\hline & Left (BS) & 24 & $a$ & 133 & 20 & 183 & -505 & 57 & -633 & 62 & 2 & 0.26 & 0.03 \\
\hline & & & $\mathrm{a} / \mathrm{m}$ & 147 & 21 & 201 & -512 & 56 & -634 & 63 & 2 & 0.29 & 0.03 \\
\hline & & & $\mathrm{m}$ & 153 & 22 & 211 & -509 & 56 & -628 & 64 & 2 & 0.3 & 0.03 \\
\hline & & & $\mathrm{m} / \mathrm{p}$ & 155 & 22 & 215 & -506 & 55 & -623 & 65 & 3 & 0.31 & 0.03 \\
\hline & & & $p$ & 152 & 22 & 214 & -481 & 54 & -594 & 66 & 3 & 0.32 & 0.04 \\
\hline 3 Ventral & Right (WS) & 18 & $a$ & 83 & 20 & 126 & -473 & 76 & -561 & 61 & 5 & 0.18 & 0.05 \\
\hline angular & & & $\mathrm{a} / \mathrm{m}$ & 74 & 12 & 98 & -443 & 66 & -525 & 57 & 6 & 0.17 & 0.04 \\
\hline & & & $\mathrm{m}$ & 32 & 10 & 54 & -309 & 51 & -374 & 56 & 6 & 0.1 & 0.04 \\
\hline & & & $\mathrm{m} / \mathrm{p}$ & 11 & 7 & 23 & -224 & 46 & -279 & 57 & 4 & 0.05 & 0.03 \\
\hline & & & $p$ & 126 & 82 & 289 & -112 & 30 & -177 & -33 & 9 & 1.27 & 0.13 \\
\hline & Left (BS) & 21 & $a$ & 117 & 29 & 169 & -621 & 143 & -853 & 53 & 4 & 0.2 & 0.04 \\
\hline & & & $\mathrm{a} / \mathrm{m}$ & 116 & 32 & 177 & -611 & 151 & -859 & 52 & 4 & 0.19 & 0.05 \\
\hline & & & $\mathrm{m}$ & 106 & 36 & 174 & -584 & 160 & -846 & 52 & 4 & 0.18 & 0.05 \\
\hline & & & $\mathrm{m} / \mathrm{p}$ & 99 & 37 & 170 & -567 & 165 & -836 & 53 & 5 & 0.18 & 0.05 \\
\hline & & & $p$ & 84 & 36 & 154 & -528 & 172 & -795 & 55 & 5 & 0.16 & 0.05 \\
\hline 4 Lateral & Right (WS) & 21 & a & 116 & 55 & 203 & -88 & 25 & -154 & 79 & 14 & 1.55 & 0.92 \\
\hline dentary & & & $\mathrm{a} / \mathrm{m}$ & 130 & 33 & 188 & -158 & 37 & -229 & 90 & 8 & 0.9 & 0.39 \\
\hline & & & $\mathrm{m}$ & 58 & 7 & 74 & -231 & 37 & -288 & -68 & 3 & 0.25 & 0.05 \\
\hline & & & $\mathrm{m} / \mathrm{p}$ & 64 & 10 & 81 & -327 & 52 & -404 & -57 & 5 & 0.2 & 0.03 \\
\hline & & & $\mathrm{p}$ & 128 & 27 & 188 & -322 & 84 & -459 & -44 & 4 & 0.41 & 0.11 \\
\hline & Left (BS) & 23 & $\mathrm{a}$ & 289 & 96 & 426 & -205 & 39 & -293 & 38 & 8 & 1.47 & 0.51 \\
\hline & & & $\mathrm{a} / \mathrm{m}$ & 349 & 74 & 490 & -226 & 45 & -330 & 37 & 7 & 1.61 & 0.52 \\
\hline & & & $\mathrm{m}$ & 387 & 77 & 532 & -234 & 49 & -347 & 36 & 7 & 1.73 & 0.54 \\
\hline & & & $\mathrm{m} / \mathrm{p}$ & 403 & 79 & 550 & -237 & 51 & -354 & 35 & 7 & 1.78 & 0.55 \\
\hline & & & $\mathrm{p}$ & 414 & 78 & 560 & -229 & 51 & -348 & 34 & 6 & 1.9 & 0.58 \\
\hline 5 Ventral & Right (WS) & 22 & $a$ & 65 & 10 & 84 & -738 & 68 & -900 & 64 & 3 & 0.09 & 0.01 \\
\hline dentary & & & $\mathrm{a} / \mathrm{m}$ & 53 & 12 & 78 & -571 & 51 & -709 & 74 & 3 & 0.09 & 0.02 \\
\hline & & & $\mathrm{m}$ & 98 & 34 & 167 & -200 & 23 & -263 & -80 & 5 & 0.5 & 0.19 \\
\hline & & & $\mathrm{m} / \mathrm{p}$ & 342 & 93 & 565 & -102 & 23 & -165 & -50 & 3 & 3.39 & 0.74 \\
\hline & & & $p$ & 674 & 92 & 938 & -103 & 19 & -142 & -34 & 3 & 7 & 0.72 \\
\hline & Left (BS) & 21 & $a$ & 253 & 19 & 288 & -838 & 118 & -1051 & 75 & 3 & 0.31 & 0.04 \\
\hline & & & $\mathrm{a} / \mathrm{m}$ & 282 & 20 & 322 & -847 & 121 & -1067 & 74 & 3 & 0.34 & 0.05 \\
\hline & & & $\mathrm{m}$ & 295 & 21 & 337 & -843 & 121 & -1066 & 73 & 3 & 0.36 & 0.05 \\
\hline & & & $\mathrm{m} / \mathrm{p}$ & 299 & 22 & 343 & -839 & 121 & -1062 & 73 & 3 & 0.36 & 0.05 \\
\hline & & & $\mathrm{p}$ & 291 & 22 & 335 & -795 & 115 & -1010 & 72 & 3 & 0.37 & 0.05 \\
\hline
\end{tabular}


Table 7. (continued)

\begin{tabular}{|c|c|c|c|c|c|c|c|c|c|c|c|c|c|}
\hline \multirow[b]{2}{*}{ Gage ID } & \multirow[b]{2}{*}{ Gage side } & \multirow{2}{*}{$\begin{array}{l}\text { No. of } \\
\text { bricks }\end{array}$} & \multirow{2}{*}{$\begin{array}{l}\text { Bite } \\
\text { position* }\end{array}$} & \multicolumn{3}{|c|}{ E1 } & \multicolumn{3}{|c|}{ E2 } & \multicolumn{2}{|c|}{$\begin{array}{c}\text { E1 } \\
\text { orientation }\end{array}$} & \multicolumn{2}{|c|}{ E1/E2 ratio } \\
\hline & & & & Mean & SD & Max & Mean & SD & Max & Mean & SD & Mean & SD \\
\hline \multirow{10}{*}{$\begin{array}{r}6 \text { Medial } \\
\text { splenial }\end{array}$} & Right (WS) & 24 & $a$ & 63 & 14 & 89 & -277 & 32 & -334 & 116 & 8 & 0.23 & 0.05 \\
\hline & & & $\mathrm{a} / \mathrm{m}$ & 63 & 15 & 97 & -179 & 20 & -220 & 122 & 9 & 0.35 & 0.09 \\
\hline & & & $\mathrm{m}$ & 190 & 19 & 225 & -140 & 12 & -167 & 142 & 2 & 1.43 & 0.17 \\
\hline & & & $\mathrm{m} / \mathrm{p}$ & 312 & 27 & 388 & -135 & 33 & -181 & 147 & 3 & 2.49 & 0.77 \\
\hline & & & $\mathrm{p}$ & 420 & 55 & 544 & -30 & 18 & -80 & 32 & 7 & 30.68 & 51.32 \\
\hline & Left (BS) & 23 & a & 255 & 37 & 322 & -628 & 48 & -700 & 124 & 2 & 0.41 & 0.08 \\
\hline & & & $\mathrm{a} / \mathrm{m}$ & 295 & 41 & 370 & -683 & 53 & -765 & 125 & 2 & 0.44 & 0.08 \\
\hline & & & $\mathrm{m}$ & 321 & 43 & 401 & -721 & 57 & -808 & 125 & 2 & 0.45 & 0.08 \\
\hline & & & $\mathrm{m} / \mathrm{p}$ & 333 & 45 & 416 & -738 & 58 & -828 & 125 & 2 & 0.46 & 0.08 \\
\hline & & & $p$ & 351 & 46 & 438 & -751 & 60 & -844 & 125 & 2 & 0.47 & 0.09 \\
\hline \multirow{10}{*}{$\begin{array}{l}7 \mathrm{DL} \\
\text { dentary }\end{array}$} & Right (WS) & 25 & $a$ & 490 & 64 & 626 & -56 & 18 & -93 & 1 & 3 & 9.38 & 2.37 \\
\hline & & & $\mathrm{a} / \mathrm{m}$ & 396 & 47 & 496 & -45 & 16 & -81 & -6 & 4 & 9.66 & 3.47 \\
\hline & & & $\mathrm{m}$ & 112 & 11 & 128 & -44 & 10 & -74 & -28 & 8 & 2.64 & 0.62 \\
\hline & & & $\mathrm{m} / \mathrm{p}$ & 40 & 17 & 70 & -158 & 26 & -214 & -68 & 2 & 0.26 & 0.11 \\
\hline & & & $\mathrm{p}$ & 412 & 91 & 543 & -707 & 122 & -1000 & -76 & 5 & 0.61 & 0.2 \\
\hline & Left (BS) & 18 & a & 472 & 109 & 556 & -146 & 30 & -192 & 1 & 4 & 3.25 & 0.93 \\
\hline & & & $\mathrm{a} / \mathrm{m}$ & 503 & 111 & 581 & -166 & 36 & -221 & 0 & 3 & 3.06 & 0.84 \\
\hline & & & $\mathrm{m}$ & 527 & 117 & 610 & -183 & 37 & -241 & 0 & 3 & 2.93 & 0.84 \\
\hline & & & $\mathrm{m} / \mathrm{p}$ & 539 & 119 & 625 & -191 & 38 & -251 & 0 & 3 & 2.87 & 0.82 \\
\hline & & & $\mathrm{p}$ & 547 & 121 & 634 & -201 & 36 & -262 & -1 & 4 & 2.77 & 0.82 \\
\hline \multirow{10}{*}{$\begin{array}{l}8 \mathrm{DL} \\
\text { dentary }\end{array}$} & Right (WS) & 23 & a & 401 & 53 & 492 & -34 & 11 & -56 & 0 & 2 & 12.96 & 4.4 \\
\hline & & & $\mathrm{a} / \mathrm{m}$ & 357 & 47 & 434 & -16 & 10 & -40 & -6 & 3 & 47 & 64 \\
\hline & & & $\mathrm{m}$ & 149 & 20 & 181 & -6 & 3 & -12 & -18 & 8 & 41 & 31 \\
\hline & & & $\mathrm{m} / \mathrm{p}$ & 57 & 13 & 80 & -32 & 13 & -56 & -57 & 7 & 2.12 & 1.12 \\
\hline & & & $\mathrm{p}$ & 615 & 66 & 720 & -599 & 107 & -786 & -83 & 3 & 1.06 & 0.23 \\
\hline & Left (BS) & 22 & a & 426 & 50 & 508 & -140 & 18 & -180 & -52 & 1 & 3.09 & 0.57 \\
\hline & & & $\mathrm{a} / \mathrm{m}$ & 451 & 51 & 534 & -165 & 21 & -212 & -54 & 1 & 2.78 & 0.51 \\
\hline & & & $\mathrm{m}$ & 477 & 51 & 559 & -183 & 23 & -233 & -55 & 1 & 2.66 & 0.46 \\
\hline & & & $\mathrm{m} / \mathrm{p}$ & 491 & 51 & 573 & -192 & 24 & -244 & -55 & 1 & 2.6 & 0.45 \\
\hline & & & $\mathrm{p}$ & 508 & 50 & 586 & -200 & 25 & -253 & -55 & 2 & 2.58 & 0.42 \\
\hline \multirow{10}{*}{$\begin{array}{r}9 \text { Lateral } \\
\text { dentary }\end{array}$} & Right (WS) & 24 & $a$ & 44 & 26 & 100 & -63 & 14 & -93 & -46 & 11 & 0.74 & 0.43 \\
\hline & & & $\mathrm{a} / \mathrm{m}$ & 89 & 27 & 144 & -105 & 16 & -140 & -50 & 6 & 0.88 & 0.32 \\
\hline & & & $\mathrm{m}$ & 82 & 14 & 108 & -118 & 15 & -146 & -62 & 3 & 0.72 & 0.19 \\
\hline & & & $\mathrm{m} / \mathrm{p}$ & 89 & 11 & 108 & -137 & 11 & -154 & -70 & 1 & 0.66 & 0.12 \\
\hline & & & $p$ & 307 & 61 & 414 & -47 & 29 & -105 & -92 & 3 & 22 & 55 \\
\hline & Left (BS) & 20 & a & 156 & 25 & 192 & -180 & 15 & -211 & 38 & 5 & 0.87 & 0.14 \\
\hline & & & $\mathrm{a} / \mathrm{m}$ & 187 & 27 & 225 & -206 & 17 & -244 & 37 & 4 & 0.91 & 0.13 \\
\hline & & & $\mathrm{m}$ & 212 & 28 & 255 & -217 & 19 & -261 & 35 & 4 & 0.98 & 0.13 \\
\hline & & & $\mathrm{m} / \mathrm{p}$ & 226 & 29 & 270 & -222 & 20 & -268 & 34 & 3 & 1.02 & 0.13 \\
\hline & & & $\mathrm{p}$ & 245 & 31 & 292 & -220 & 21 & -268 & 33 & 3 & 1.12 & 0.14 \\
\hline \multirow{10}{*}{$\begin{array}{l}10 \text { Ventral } \\
\text { dentary }\end{array}$} & Right (WS) & 21 & $a$ & 69 & 9 & 86 & -464 & 43 & -566 & 58 & 2 & 0.15 & 0.02 \\
\hline & & & $\mathrm{a} / \mathrm{m}$ & 55 & 6 & 65 & -409 & 35 & -495 & 62 & 2 & 0.13 & 0.02 \\
\hline & & & $\mathrm{m}$ & 27 & 4 & 34 & -214 & 18 & -249 & 67 & 2 & 0.13 & 0.02 \\
\hline & & & $\mathrm{m} / \mathrm{p}$ & 21 & 9 & 46 & -84 & 15 & -113 & 86 & 9 & 0.26 & 0.12 \\
\hline & & & $\mathrm{p}$ & 350 & 38 & 439 & -40 & 11 & -58 & -32 & 2 & 9.54 & 4.06 \\
\hline & Left (BS) & 23 & a & 142 & 10 & 158 & -613 & 64 & -711 & 45 & 1 & 0.23 & 0.02 \\
\hline & & & $\mathrm{a} / \mathrm{m}$ & 149 & 10 & 165 & -612 & 65 & -716 & 43 & 1 & 0.25 & 0.02 \\
\hline & & & $\mathrm{m}$ & 152 & 10 & 169 & -606 & 67 & -714 & 42 & 1 & 0.25 & 0.03 \\
\hline & & & $\mathrm{m} / \mathrm{p}$ & 153 & 10 & 171 & -601 & 67 & -712 & 42 & 1 & 0.26 & 0.03 \\
\hline & & & $\mathrm{p}$ & 149 & 10 & 167 & -572 & 66 & -680 & 41 & 2 & 0.26 & 0.03 \\
\hline
\end{tabular}


Table 7. (continued)

\begin{tabular}{|c|c|c|c|c|c|c|c|c|c|c|c|c|c|}
\hline \multirow[b]{2}{*}{ Gage ID } & \multirow[b]{2}{*}{ Gage side } & \multirow{2}{*}{$\begin{array}{l}\text { No. of } \\
\text { bricks }\end{array}$} & \multirow{2}{*}{$\begin{array}{l}\text { Bite } \\
\text { position* }\end{array}$} & \multicolumn{3}{|c|}{ E1 } & \multicolumn{3}{|c|}{ E2 } & \multicolumn{2}{|c|}{$\begin{array}{c}\text { E1 } \\
\text { orientation }\end{array}$} & \multicolumn{2}{|c|}{ E1/E2 ratio } \\
\hline & & & & Mean & SD & Max & Mean & SD & Max & Mean & SD & Mean & SD \\
\hline \multirow{10}{*}{$\begin{array}{l}11 \text { Ventral } \\
\text { splenial }\end{array}$} & Right (WS) & 22 & $\mathrm{a}$ & 133 & 12 & 156 & -593 & 33 & -637 & 55 & 2 & 0.22 & 0.02 \\
\hline & & & $\mathrm{a} / \mathrm{m}$ & 111 & 14 & 138 & -474 & 28 & -512 & 57 & 3 & 0.23 & 0.03 \\
\hline & & & $\mathrm{m}$ & 49 & 9 & 67 & -187 & 20 & -216 & 59 & 3 & 0.27 & 0.07 \\
\hline & & & $\mathrm{m} / \mathrm{p}$ & 26 & 13 & 60 & -8 & 5 & -16 & -60 & 20 & 6.13 & 6.96 \\
\hline & & & $p$ & 524 & 29 & 569 & -127 & 15 & -158 & -37 & 2 & 4.16 & 0.46 \\
\hline & Left (BS) & 22 & $\mathrm{a}$ & 169 & 17 & 201 & -789 & 82 & -922 & -46 & 2 & 0.21 & 0.02 \\
\hline & & & $\mathrm{a} / \mathrm{m}$ & 175 & 19 & 210 & -806 & 82 & -942 & -45 & 2 & 0.22 & 0.02 \\
\hline & & & $\mathrm{m}$ & 179 & 20 & 217 & -822 & 81 & -956 & -44 & 2 & 0.22 & 0.02 \\
\hline & & & $\mathrm{m} / \mathrm{p}$ & 181 & 20 & 219 & -829 & 80 & -962 & -44 & 2 & 0.22 & 0.02 \\
\hline & & & $\mathrm{p}$ & 179 & 19 & 217 & -822 & 77 & -948 & -45 & 2 & 0.22 & 0.02 \\
\hline \multirow{10}{*}{$\begin{array}{l}12 \text { Medial } \\
\text { splenial }\end{array}$} & Right (WS) & 22 & $a$ & 37 & 24 & 87 & -77 & 28 & -132 & -50 & 14 & 0.59 & 0.5 \\
\hline & & & $\mathrm{a} / \mathrm{m}$ & 62 & 32 & 127 & -46 & 16 & -77 & -32 & 12 & 1.58 & 1.03 \\
\hline & & & $\mathrm{m}$ & 174 & 15 & 202 & -86 & 9 & -102 & -36 & 3 & 2.08 & 0.27 \\
\hline & & & $\mathrm{m} / \mathrm{p}$ & 257 & 20 & 295 & -116 & 8 & -130 & -38 & 1 & 2.21 & 0.13 \\
\hline & & & $p$ & 157 & NA & 157 & -6 & NA & -6 & 55 & NA & 24.31 & NA \\
\hline & Left (BS) & 20 & $a$ & 212 & 25 & 259 & -298 & 64 & -445 & -54 & 3 & 0.75 & 0.22 \\
\hline & & & $a / m$ & 246 & 25 & 293 & -340 & 68 & -493 & -55 & 3 & 0.76 & 0.2 \\
\hline & & & $\mathrm{m}$ & 269 & 26 & 315 & -369 & 72 & -527 & -55 & 2 & 0.76 & 0.19 \\
\hline & & & $\mathrm{m} / \mathrm{p}$ & 281 & 26 & 327 & -383 & 73 & -542 & -56 & 2 & 0.76 & 0.18 \\
\hline & & & $p$ & 299 & 26 & 344 & -398 & 74 & -555 & -56 & 2 & 0.78 & 0.18 \\
\hline \multirow{10}{*}{$\begin{array}{l}13 \text { Lateral } \\
\text { dentary }\end{array}$} & Right (WS) & 25 & $a$ & 105 & 33 & 184 & -56 & 9 & -83 & -30 & 6 & 1.89 & 0.6 \\
\hline & & & $\mathrm{a} / \mathrm{m}$ & 129 & 25 & 196 & -82 & 10 & -112 & -40 & 4 & 1.62 & 0.43 \\
\hline & & & $\mathrm{m}$ & 78 & 9 & 98 & -86 & 8 & -98 & -62 & 2 & 0.93 & 0.15 \\
\hline & & & $\mathrm{m} / \mathrm{p}$ & 79 & 6 & 94 & -122 & 14 & -159 & -78 & 2 & 0.66 & 0.08 \\
\hline & & & $\mathrm{p}$ & 300 & 54 & 410 & -178 & 44 & -281 & 86 & 2 & 1.74 & 0.32 \\
\hline & Left (BS) & 24 & $\mathrm{a}$ & 192 & 24 & 237 & -167 & 24 & -227 & 29 & 6 & 1.18 & 0.27 \\
\hline & & & $a / m$ & 226 & 24 & 267 & -197 & 25 & -262 & 29 & 5 & 1.17 & 0.23 \\
\hline & & & $\mathrm{m}$ & 252 & 25 & 296 & -211 & 24 & -278 & 28 & 5 & 1.21 & 0.21 \\
\hline & & & $m / p$ & 265 & 26 & 308 & -218 & 24 & -285 & 28 & 5 & 1.23 & 0.21 \\
\hline & & & $p$ & 279 & 26 & 324 & -217 & 23 & -281 & 27 & 4 & 1.3 & 0.21 \\
\hline \multirow{10}{*}{$\begin{array}{l}14 \text { Medial } \\
\text { splenial }\end{array}$} & Right (WS) & 22 & $a$ & 96 & 35 & 173 & -127 & 35 & -202 & -21 & 5 & 0.75 & 0.16 \\
\hline & & & $a / m$ & 97 & 35 & 177 & -89 & 27 & -149 & -16 & 7 & 1.1 & 0.27 \\
\hline & & & $\mathrm{m}$ & 80 & 13 & 106 & -17 & 5 & -24 & -67 & 12 & 5.27 & 3.31 \\
\hline & & & $\mathrm{m} / \mathrm{p}$ & 166 & 14 & 190 & -63 & 20 & -92 & 96 & 2 & 3.02 & 1.43 \\
\hline & & & $p$ & 378 & 47 & 463 & -58 & 26 & -115 & 94 & 4 & 8.46 & 5.09 \\
\hline & Left (BS) & 25 & $a$ & 153 & 25 & 207 & -244 & 38 & -314 & 121 & 2 & 0.63 & 0.07 \\
\hline & & & $\mathrm{a} / \mathrm{m}$ & 169 & 23 & 218 & -277 & 39 & -349 & 118 & 2 & 0.62 & 0.07 \\
\hline & & & $\mathrm{m}$ & 186 & 23 & 236 & -310 & 40 & -387 & 118 & 2 & 0.6 & 0.07 \\
\hline & & & $\mathrm{m} / \mathrm{p}$ & 196 & 23 & 247 & -328 & 41 & -406 & 117 & 2 & 0.6 & 0.07 \\
\hline & & & $p$ & 216 & 26 & 271 & -358 & 42 & -439 & 118 & 2 & 0.61 & 0.06 \\
\hline
\end{tabular}

a, anterior; a/m, anterior/middle; $\mathrm{m}$, middle; $\mathrm{m} / \mathrm{p}$, middle/posterior; posterior.

Strain fields of the medial aspect of the mandible (Fig. 4) reveal strong local variations in principal strain on the working side during posterior biting.

Despite (or because of) the high variability of $\varepsilon_{1}$ orientations recorded at medial gages in vivo (especially during ipsilateral biting), strain orientations predicted by the FEM overlapped the range of in vivo $\varepsilon_{1}$ orientations during ipsilateral biting (Experiments 75, 102, 103) and contralateral biting (Experiment 99), and were similar to $\varepsilon_{1}$ orientations recorded during contralateral biting in Experiments 75, 102 and 103.
FEM strain orientations did not fall within the range of data recorded at the medial gage site in Experiment 99 during ipsilateral biting, despite the fact that in vivo $\varepsilon_{1}$ orientations at this site were highly variable (spanning over $115^{\circ}$ ).

\section{Principal strain $\left(\varepsilon_{1}\right)$ orientations: ventral mandible}

In vivo strain orientations

On the ventral aspect of the working side, $\varepsilon_{1}$ strain orientation is highly variable between experiments and gage sites 


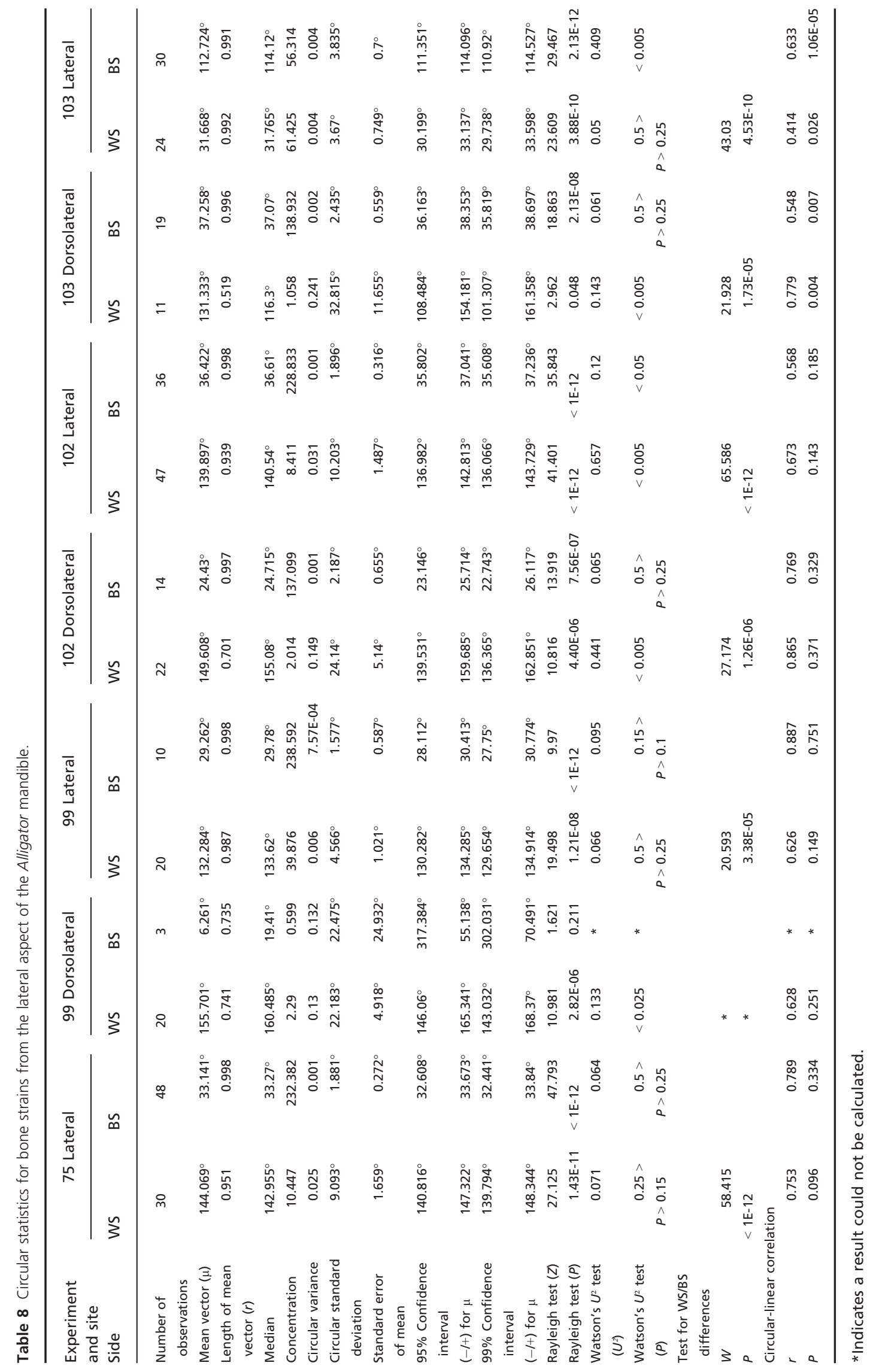


Table 9 Circular statistics for bone strains from the medial aspect of the Alligator mandible.

\begin{tabular}{|c|c|c|c|c|c|c|c|c|}
\hline \multirow{2}{*}{$\begin{array}{l}\text { Experiment and site } \\
\text { Side }\end{array}$} & \multicolumn{2}{|c|}{75 Medial } & \multicolumn{2}{|c|}{99 Medial } & \multicolumn{2}{|c|}{102 Medial } & \multicolumn{2}{|c|}{103 Medial } \\
\hline & ws & BS & WS & BS & WS & BS & WS & BS \\
\hline No. of observations & 30 & 48 & 20 & 10 & 47 & 35 & 15 & 27 \\
\hline Mean vector $(\mu)$ & $113.66^{\circ}$ & $111.33^{\circ}$ & $86.855^{\circ}$ & $134.901^{\circ}$ & $84.21^{\circ}$ & $165.116^{\circ}$ & $99.913^{\circ}$ & $99.373^{\circ}$ \\
\hline $\begin{array}{l}\text { Length of mean } \\
\text { vector }(r)\end{array}$ & 0.994 & 0.918 & 0.302 & 0.997 & 0.634 & 0.988 & 0.064 & 0.999 \\
\hline Median & $112.99^{\circ}$ & $111.335^{\circ}$ & $95.02^{\circ}$ & $135.22^{\circ}$ & $92.36^{\circ}$ & $164.04^{\circ}$ & $93.55^{\circ}$ & $99.26^{\circ}$ \\
\hline Concentration & 90.202 & 6.401 & 0.634 & 109.854 & 1.655 & 43.003 & 0 & 879.798 \\
\hline Circular variance & 0.003 & 0.041 & 0.349 & 0.002 & 0.183 & 0.006 & 0.468 & $2.84 \mathrm{E}-04$ \\
\hline $\begin{array}{l}\text { Circular standard } \\
\text { deviation }\end{array}$ & $3.025^{\circ}$ & $11.829^{\circ}$ & $44.322^{\circ}$ & $2.326^{\circ}$ & $27.362^{\circ}$ & $4.394^{\circ}$ & $67.173^{\circ}$ & $0.966^{\circ}$ \\
\hline Standard error of mean & $0.552^{\circ}$ & $1.705^{\circ}$ & $14.636^{\circ}$ & $0.866^{\circ}$ & $4.08^{\circ}$ & $0.743^{\circ}$ & * & $0.186^{\circ}$ \\
\hline $\begin{array}{l}95 \% \text { Confidence } \\
\text { interval }\end{array}$ & $112.577^{\circ}$ & $107.987^{\circ}$ & $58.163^{\circ}$ & $133.204^{\circ}$ & $76.21^{\circ}$ & $163.66^{\circ}$ & * & $99.009^{\circ}$ \\
\hline$(-/+)$ for $\mu$ & $114.743^{\circ}$ & $114.673^{\circ}$ & $115.548^{\circ}$ & $136.598^{\circ}$ & $92.209^{\circ}$ & $166.572^{\circ}$ & * & $99.738^{\circ}$ \\
\hline $\begin{array}{l}99 \% \text { Confidence } \\
\text { interval }\end{array}$ & $112.237^{\circ}$ & $106.936^{\circ}$ & $49.15^{\circ}$ & $132.671^{\circ}$ & $73.698^{\circ}$ & $163.203^{\circ}$ & * & $98.894^{\circ}$ \\
\hline$(-/+)$ for $\mu$ & $115.083^{\circ}$ & $115.724^{\circ}$ & $124.561^{\circ}$ & $137.132^{\circ}$ & $94.721^{\circ}$ & $167.03^{\circ}$ & * & $99.852^{\circ}$ \\
\hline Rayleigh test $(Z)$ & 29.667 & 40.475 & 1.826 & 9.934 & 18.876 & 34.186 & 0.061 & 26.969 \\
\hline Rayleigh test $(P)$ & $1.81 \mathrm{E}-12$ & $<1 \mathrm{E}-12$ & 0.162 & $<1 \mathrm{E}-12$ & $2.35 \mathrm{E}-09$ & $<1 \mathrm{E}-12$ & 0.942 & $2.09 \mathrm{E}-11$ \\
\hline Watson's $U^{2}$ test $\left(U^{2}\right)$ & 0.15 & 2.292 & 0.112 & 0.048 & 0.556 & 0.361 & * & 0.186 \\
\hline Watson's $U^{2}$ test $(P)$ & $<0.025$ & $<0.005$ & $<0.005$ & $>0.5$ & $<0.005$ & $<0.005$ & * & $<0.005$ \\
\hline \multicolumn{9}{|l|}{ Test for WS/BS differences } \\
\hline$w$ & 8.422 & & 12.759 & & 64.427 & & $5.66 \mathrm{E}-04$ & \\
\hline$P$ & 0.015 & & $1.00 \mathrm{E}-03$ & & $<1 \mathrm{E}-12$ & & $9.81 \mathrm{E}-01$ & \\
\hline \multicolumn{9}{|l|}{ Circular-linear correlation } \\
\hline$r$ & 0.559 & 0.593 & 0.698 & 0.998 & 0.462 & 0.700 & 0.972 & 0.668 \\
\hline$P$ & 0.378 & 0.454 & 0.080 & 0.537 & 0.271 & 0.091 & 0.349 & 0.244 \\
\hline
\end{tabular}

*Indicates a result could not be calculated.

(Fig. 2, Tables 2-6), directed anteromedially [Experiments 56 (anterior and posterior dentary gages), 75, 99, and 102 (ventral splenial gage)] or anterolaterally [Experiments 56 (angular gage), 102 (ventral dentary gage), and 103]. During ipsilateral biting, $\varepsilon_{1}$ orientations at ventral gage sites tend to rotate in a counterclockwise direction (relative to the reference axis) as bite point moves posteriorly.

With the exception of Experiment 56, $\varepsilon_{1}$ on the ventral aspect of the mandible during contralateral biting is nearly perpendicular to the reference axis (Fig. 3, Tables 2-6). In Experiment 56, principal strains are parallel to the reference axis. Contralateral biting does not produce any obvious trend of change in $\varepsilon_{1}$ orientation with change in bite point. At most ventral gage sites, $\varepsilon_{1}$ strains are more concentrated during contralateral than ipsilateral biting (Table 10). The exceptions are the posterior dentary and angular gages of Experiment 56, in which the reverse is true.

In all experiments, ventral $\varepsilon_{1}$ orientations during ipsilateral bites are significantly different from those recorded during contralateral biting (Table 10). Two-way ANovA (Table 11) suggests that differences in bite point and gage sites/experimental animals have a significant impact on $\varepsilon_{1}$ orientations. There appears to be some correlation between strain orientation and magnitude at ventral gage sites but these are rarely significant (Table 10).

\section{FEM strain orientations}

Working side strain orientations at all ventral gage sites are directed anterolaterally during anterior bites to anteromedially during posterior bites (Fig. 2, Table 7). On the balancing side (Fig. 3, Table 7) $\varepsilon_{1}$ orientations are directed perpendicular to the reference axis at more anterior gage sites [Experiments 56 (anterior dentary), 75, 99 and 103] but anterolaterally or anteromedially at more posterior gage sites [Experiments 56 (posterior dentary and angular sites) and 102]. Within balancing side gage sites, $\varepsilon_{1}$ orientations varied by no more than $5^{\circ}$ with changes in bite point. As with lateral and medial gage sites, ventral strain orientations in the FEM vary less with changes in bite point on the balancing side (Fig. 3) than on the working side (Figs 2 and 4). Unlike the lateral and medial aspects of the mandible, strain orientations are more variable between ventral gage sites during middle biting than during posterior biting (Fig. 4). This may be due to the more anterior location of ventral gages (thus, orientations reflect the proximity of bite point). Alternatively, high variability in 


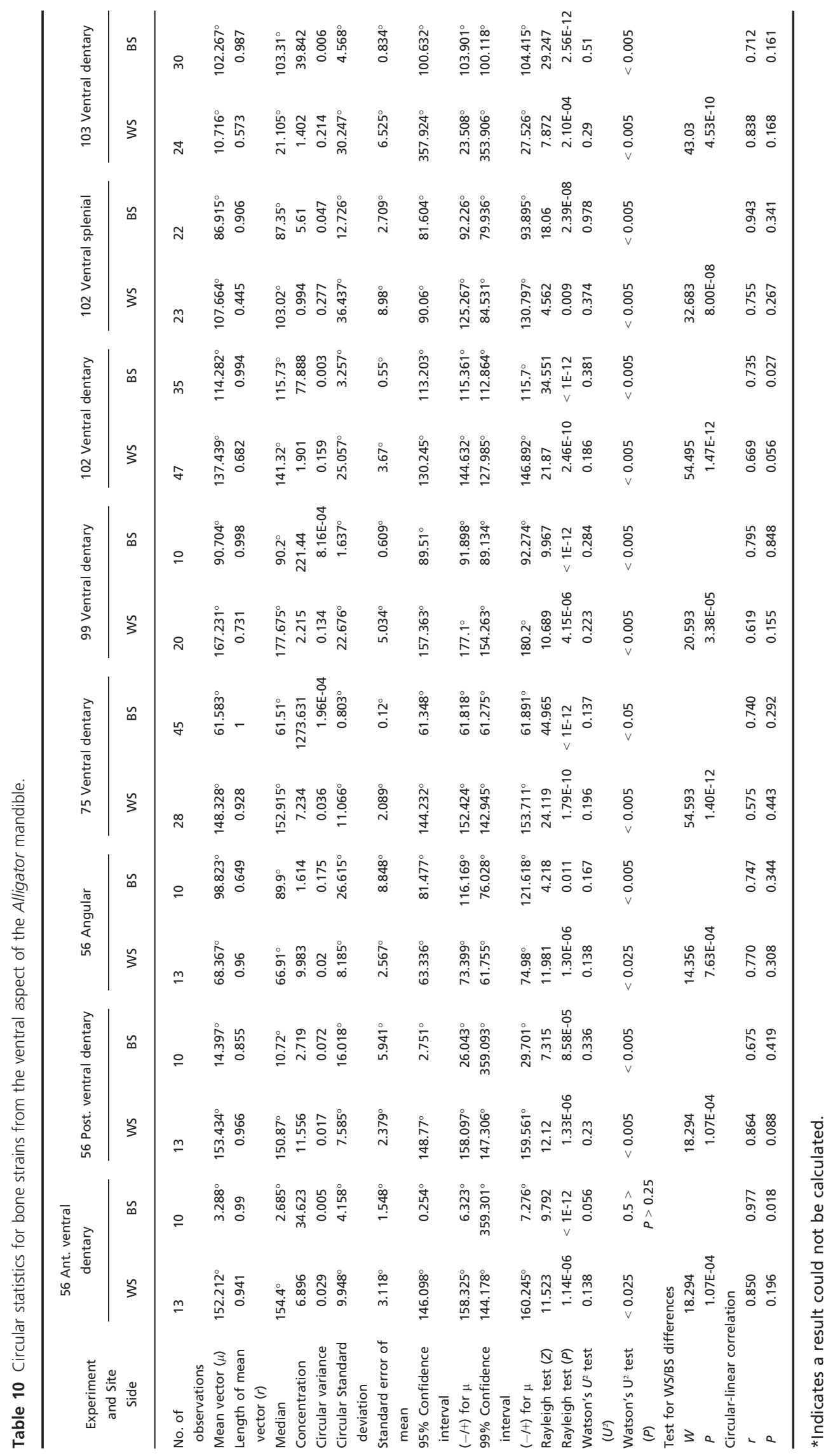


Table 11 Two-way ANOva comparing variation within individual gage sites or between research subjects ('Individual') and with changes in bite location ('Bite Point') in four regions of the Alligator mandible.

\begin{tabular}{|c|c|c|c|c|c|c|c|}
\hline \multicolumn{4}{|c|}{ Dorsolateral WS } & \multicolumn{4}{|c|}{ Dorsolateral BS } \\
\hline Source & df & $\mathrm{CHI} 2$ & $P$-value & Source & df & $F$ & $P$-value \\
\hline 'Individual' & 4 & 7.96897 & 0.092721 & 'Individual' & 2 & 17.46796 & 0.003149 \\
\hline 'Bite Point' & 6 & 41.2755 & $2.56 \mathrm{E}-07$ & 'Bite Point' & 3 & 3.814009 & 0.076628 \\
\hline \multicolumn{4}{|c|}{ Lateral WS } & \multicolumn{4}{|c|}{ Lateral BS } \\
\hline Source & df & $\mathrm{CHI} 2$ & $P$-value & Source & $d f$ & $\mathrm{CHI} 2$ & $P$-value \\
\hline 'Individual' & 6 & 211.6827 & 0 & 'Individual' & 6 & 244.7956 & 0 \\
\hline 'Bite Point' & 8 & 16.09427 & 0.04105 & 'Bite Point' & 8 & 16.38079 & 0.037243 \\
\hline \multicolumn{4}{|c|}{ Medial WS } & \multicolumn{4}{|c|}{ Medial BS } \\
\hline Source & $' d f '$ & 'CHI2' & 'P-value' & Source & 'df' & 'CHI2' & 'P-value' \\
\hline 'Individual' & 6 & 54.52522 & $5.78 \mathrm{E}-10$ & 'Individual' & 6 & 213.2399 & 0 \\
\hline 'Bite Point' & 8 & 71.97532 & $1.98 \mathrm{E}-12$ & ‘Bite Point' & 8 & 7.993922 & 0.434064 \\
\hline \multicolumn{4}{|c|}{ Ventral WS } & \multicolumn{4}{|c|}{ Ventral BS } \\
\hline 'Source' & $' d f '$ & 'CHI2' & 'P-value' & 'Source' & 'df' & 'CHI2' & 'P-value' \\
\hline 'Individual' & 14 & 168.7355 & 0 & 'Individual' & 14 & 303.9026 & 0 \\
\hline 'Bite Point' & 8 & 48.30766 & $8.63 \mathrm{E}-08$ & 'Bite Point' & 8 & 33.75298 & 4.50E-05 \\
\hline
\end{tabular}

strain orientations on the ventral aspect of the FEM may be a result of numerous sutural contacts - note that during middle biting, there is a distinct line of reversal in strain orientation between the splenial and dentary bones.

Because of high variability in in vivo principal strain orientations on the ventral surface of the mandible during ipsilateral biting, strain orientations in the working side FEM broadly overlap in vivo strain orientations at all gage sites except the posterior dentary gage of Experiment 56. The range of strain orientations predicted by the balancing side FEM resembled in vivo $\varepsilon_{1}$ orientations recorded in Experiments 75, 99 and 103 during contralateral biting but did not match strain orientations from Experiments 56 or 102.

\section{Principal strain magnitudes and ratios}

Within-gage in vivo $\varepsilon_{1}$ means (Tables 2-6) ranged from 34 to $1679 \mu \varepsilon$ and the grand mean for all gage sites was $924 \mu \varepsilon$. Within-gage in vivo $\varepsilon_{2}$ means ranged from -145 to $-1849 \mu \varepsilon$ with a grand mean of $-955 \mu \varepsilon$ for all gage sites. There was no difference in mean $\varepsilon_{1}$ strain magnitudes on the working and balancing sides (Table 12); in contrast, mean $\varepsilon_{2}$ strain magnitudes were greater on the balancing side. During both ipsilateral and contralateral biting, in vivo principal strain magnitudes were higher in the dentary than in the splenial.
The grand $\varepsilon_{1} / \varepsilon_{2}$ mean for all in vivo experiments was 1.18 . The $\varepsilon_{1} / \varepsilon_{2}$ ratio was usually $>1$ on the working side mandible as a whole as well as in the working side dentary, but $<1$ on the balancing side mandible and dentary. These results indicate that the working side mandible (all bones) and the dentary experience predominantly tension, whereas these structures on the balancing side experience predominantly compression. The $\varepsilon_{1} / \varepsilon_{2}$ ratio was $<1$ in the splenial during both ipsilateral and contralateral biting, suggesting that the splenial is primarily under compression.

$\varepsilon_{1}$ and $\varepsilon_{2}$ strains were between two and five times higher during in vivo experiments than those exhibited by the FEM (Table 12). Like the in vivo results, the FEM exhibited higher $\varepsilon_{2}$ strains (as well as higher $\varepsilon_{1}$ strains) on the balancing side than on the working side. Unlike the in vivo results, strains were not consistently higher in the dentary of the FEM than in the splenial. $\varepsilon_{1} / \varepsilon_{2}$ ratios in the FEM were always higher on the working side than on the balancing side. Unlike the in vivo experiments, in which the splenial was always in compression, only the balancing side splenial of the FEM exhibited an $\varepsilon_{1} / \varepsilon_{2}$ ratio $<1$.

\section{Shear strain}

Within-gage means of in vivo maximum shear strain ranged from 197 to $3528 \mu \varepsilon$, the grand mean for all gage sites being 
Table 12 Mean shear and principal strain magnitudes across all bite points for different region of the mandible, in vivo and in silico. Bold values indicate significant results.

\begin{tabular}{|c|c|c|c|c|c|c|c|}
\hline & \multicolumn{4}{|c|}{ In vivo experiments } & \multicolumn{3}{|c|}{ FEM } \\
\hline & \multicolumn{4}{|c|}{ Shear strain } & \multicolumn{3}{|c|}{ Shear strain } \\
\hline & Grand mean & WS mean & BS mean & $P$-value & Grand mean & WS mean & BS mean \\
\hline All gage sites & 1879 & 1630 & $<2130$ & $<0.001$ & 518 & 339 & $<696$ \\
\hline Dentary & 2122 & 1809 & $<2449$ & $<0.001$ & 504 & 346 & $<661$ \\
\hline \multirow[t]{3}{*}{ Splenial } & 1373 & 1240 & $<1498$ & 0.03 & 545 & 312 & $<778$ \\
\hline & \multicolumn{4}{|c|}{ E1 } & \multicolumn{3}{|c|}{ E1 } \\
\hline & Grand mean & WS mean & BS mean & $P$-value & Grand mean & WS mean & BS mean \\
\hline All gage sites & 924 & 920 & $=928$ & 0.85 & 208 & 157 & $<258$ \\
\hline Dentary & 1110 & 1083 & $=1138$ & 0.34 & 224 & 161 & $<286$ \\
\hline \multirow[t]{3}{*}{ Splenial } & 536 & 562 & $=512$ & 0.3 & 202 & 170 & $<233$ \\
\hline & \multicolumn{4}{|c|}{$\mathrm{E} 2 *$} & \multicolumn{3}{|c|}{$\mathrm{E} 2 *$} \\
\hline & Grand mean & WS mean & BS mean & $P$-value & Grand mean & WS mean & BS mean \\
\hline All gage sites & -955 & -710 & $<-1201$ & $<0.001$ & -310 & -182 & $<-438$ \\
\hline Dentary & -1012 & -725 & $<-1310$ & $<0.001$ & -280 & -185 & $<-375$ \\
\hline \multirow[t]{3}{*}{ Splenial } & -837 & -678 & $<-986$ & $<0.001$ & -343 & -141 & $<-545$ \\
\hline & \multicolumn{4}{|c|}{$\mathrm{E} 1 / \mathrm{E} 2$} & \multicolumn{3}{|c|}{$\mathrm{E} 1 / \mathrm{E} 2$} \\
\hline & Grand mean & WS mean & BS mean & $P$-value & Grand mean & WS mean & BS mean \\
\hline All gage sites & 1.18 & 1.6 & $>0.84$ & $<0.001$ & 1.95 & 2.98 & $>0.92$ \\
\hline Dentary & 1.4 & 1.88 & $>0.95$ & $<0.001$ & 2.07 & 2.95 & $>1.19$ \\
\hline Splenial & 0.77 & 0.94 & $>0.61$ & $<0.001$ & 2.1 & 3.71 & $>0.5$ \\
\hline
\end{tabular}

*More negative E2 considered to be greater (higher compressive strain).

$1879 \mu \varepsilon$ (Table 12). When all gage sites were considered, there was a significant difference between mean shear strain on the working side and balancing side (1630 vs. $2130 \mu \varepsilon$, respectively, $P<0.001)$. Similarly, when examined by region of the mandible, mean shear strain was greater on the balancing side than on the working side in both the dentary and splenial bones (Table 12). On both sides, shear strain was higher in the dentary than in the splenial. Shear strain was also higher on the balancing side in the FEM but shear was not higher in the dentary of the FEM than in the splenial. As with principal strain magnitudes, in vivo shear strains were between two and four times higher than those exhibited by the FEM.

\section{Strain gradients}

Figures 5 and 6 compare mean $\varepsilon_{1}$ and shear strain magnitudes at every gage site in vivo and in the FEM. Strain magnitudes vary greatly among in vivo gage sites. Gages on the ventral aspect of the mandible recorded both the highest
(Experiment 75) and lowest (Experiment 56) strains. Higher strains were recorded on the lateral aspect than on the medial aspect of the mandible during both ipsilateral and contralateral biting. Posterior bites generated the highest $\varepsilon_{1}$ strains at most (but not all) gage sites in the working side mandible; in contrast, middle bites tended to produce both the highest $\varepsilon_{1}$ and shear strains in the balancing side mandible.

Absolute strain magnitudes are substantially lower in the FEM than in vivo at nearly all sites (Figs 5 and 6). As with in vivo strains, the ventral aspect of the mandible featured the lowest strains (gage sites corresponding to Experiment 56) and one of the highest strains (gage site corresponding to Experiment 75); in contrast, strains on the lateral aspect of the FEM were not consistently higher than those on the medial aspect. On the working side, posterior bites usually produced the highest strains; there was little variation in strain magnitude with changes in bite location on the balancing side of the FEM. 
Table 13 ANOva testing for differences in $\varepsilon_{1}$ principal strain orientation during biting at different locations along the toothrow.

\begin{tabular}{|c|c|c|c|c|c|}
\hline Gauge location & $\begin{array}{l}\text { Experiment } \\
\text { number }\end{array}$ & $\begin{array}{l}\text { Working side bite } \\
\text { points represented* }\end{array}$ & $\begin{array}{l}P \text {-value for working } \\
\text { side differences** }\end{array}$ & $\begin{array}{l}\text { Balancing side bite } \\
\text { points represented* }\end{array}$ & $\begin{array}{l}P \text {-value for balancing } \\
\text { side differences** }\end{array}$ \\
\hline \multirow[t]{3}{*}{ Dorsolateral dentary } & 99 & $2-5$ & n.s. & 3,5 & 0.008 \\
\hline & 102 & $2-5$ & $<0.0001$ & $3-5$ & 0.007 \\
\hline & 103 & $2-5$ & 0.003 & $2-5$ & n.s. \\
\hline \multirow[t]{4}{*}{ Lateral dentary } & 75 & $2-5$ & n.s. & $1-5$ & 0.003 \\
\hline & 99 & $2-5$ & n.s. & $1,3-5$ & n.s. \\
\hline & 102 & $1-5$ & n.s. & $1-5$ & $<0.0001$ \\
\hline & 103 & $2-5$ & n.s. & $2-5$ & 0.007 \\
\hline \multirow[t]{6}{*}{ Ventral dentary } & 56 & $2-5$ & 0.016 & 1,5 & 0.007 \\
\hline & 56 & $2-5$ & 0.007 & 1,5 & n.s. \\
\hline & 75 & $2-5$ & $<0.0001$ & $1-5$ & $<0.0001$ \\
\hline & 99 & $2-5$ & $<0.0001$ & $1,3-5$ & n.s. \\
\hline & 102 & $1-5$ & $<0.0001$ & $1-5$ & n.s. \\
\hline & 103 & $2-5$ & $<0.0001$ & $2-5$ & n.s. \\
\hline Ventral angular & 56 & $2-5$ & n.s. & 1,5 & n.s. \\
\hline Ventral splenial & 102 & $1-5$ & $<0.0001$ & $1-5$ & n.s. \\
\hline \multirow[t]{4}{*}{ Medial splenial } & 75 & $2-5$ & 0.006 & $1-5$ & n.s. \\
\hline & 99 & $2-5$ & $<0.0001$ & $1,3-5$ & 0.047 \\
\hline & 102 & $1-5$ & $<0.0001$ & $1-5$ & n.s. \\
\hline & 103 & $2-5$ & $<0.0001$ & $2-5$ & n.s. \\
\hline
\end{tabular}

*Bite points 1-5 represent bite regions from anterior to posterior.

$* * P$-values represent existence of significant differences among all $\varepsilon_{1}$ principal strain orientations at bite points represented.

Contour plots of von Mises strain in the FEM reveal strain gradients throughout the mandible during biting (Fig. 7). On both sides of the lower jaw, the articulars, surangulars, ventral angulars, and bone around the external mandibular fenestrae exhibit high strains. Additionally, the ventral aspect of the balancing side ramus (dentary and splenial) experiences high strains. The working side ramus is less strained overall than the balancing side but exhibits high strain immediately below the bite point.

\section{In vivo neutral axis}

Neutral axis orientation in the working and balancing sides is presented for different bite points and experiments (Figs 8 and 9, Table 14). The orientation of the neutral axis is measured relative to the horizontal, with positive and negative angles indicating clockwise and counterclockwise rotations, respectively. Note that while the neutral axis orientation is a calculated value based on strain at individual gages, its location relative to the cortex was arbitrarily placed through the center of the cross-section.

On the working side, neutral axis orientations exhibit increased variability as bite point moves from anterior to posterior; the range of neutral axis orientations across all experiments is $68.9,98.0,118.3$ and $104.6^{\circ}$ for anterior/middle, middle, middle/posterior and posterior bite points, respectively. Although mean neutral axis orientations across bite points and experiments show some degree of consistency, they provide conflicting results in terms of bending regime. In two experiments (102 and 103), mean neutral axis orientations indicate negative dorsoventral bending (i.e. the dorsal border of the mandible under tension). However, in Experiment 75 the dorsal border is under compression. Finally, in Experiment 99 the mean neutral axis orientation indicates mediolateral bending. Maximum and minimum normal strains on the working side are similarly variable (Fig. 8, Table 14). Absolute normal strains range from $5780 \mu \varepsilon$ (anterior/middle biting, Experiment 103) to $-6130 \mu \varepsilon$ (posterior biting, Experiment 75). In two experiments (Experiment 75 and 102), maximum and minimum normal strains increase with more posterior bites, but no obvious trend exists in Experiments 99 and 103. In summary, neutral axis orientations for the working side are variable, with little consistency across experiments and large standard deviations within experiments.

In contrast, average neutral axis orientations on the balancing side show little variation, either across different bite point locations or across experiments (Fig. 9, Table 14). The exception is Experiment 99, which exhibits high variability, both within and across bite points. For Experiments 75, 102, and 103 , mean neutral axis orientation for all contralateral bite point locations ranges from 1.8 to $26.8^{\circ}$. This suggests that the balancing side consistently experiences negative dorsoventral bending (the dorsal border under tension). Additionally, there may be some lateral bending (with the lateral aspect of the mandible under tension), as indicated by the consistent counterclockwise rotation of the neutral axis. Maximum normal strains on the balancing side are similar to those on the working side but minimum normal strains are not as extreme as those recorded on the 
Working side

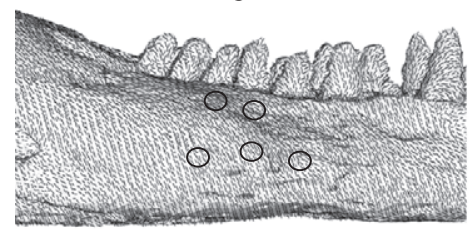

Lateral

Middle
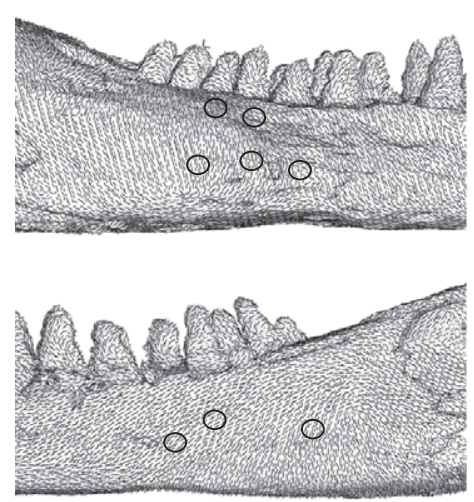

Medial

Middle

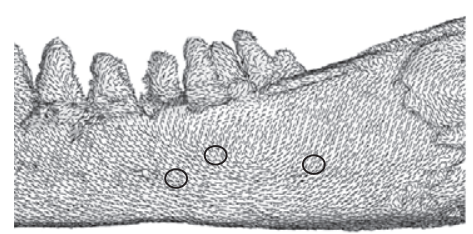

Posterior

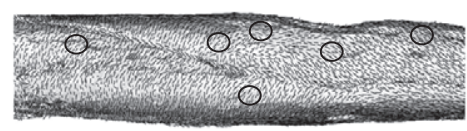

Ventral

Middle

Posterior
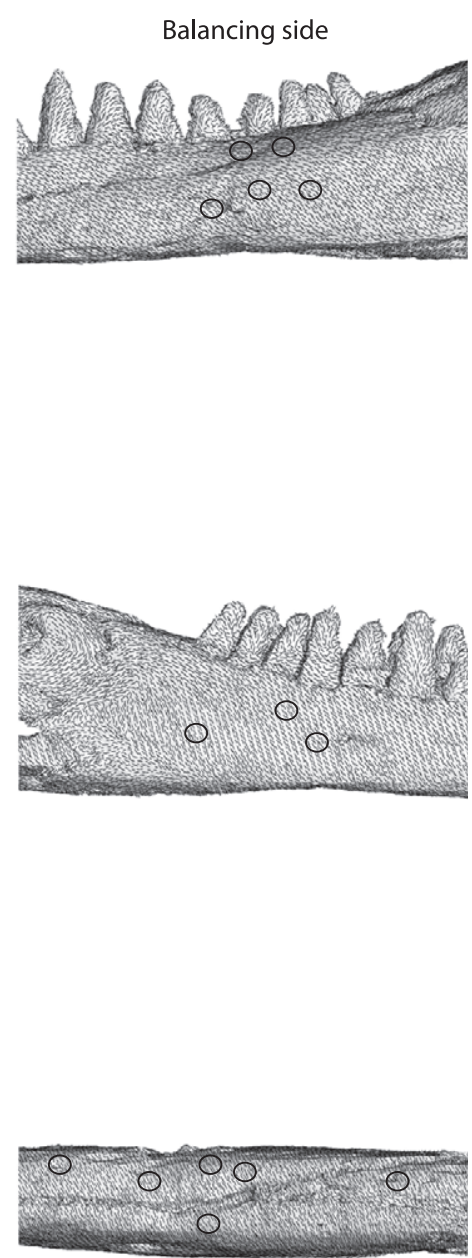

Fig. 4 Strain fields in the FEM (finite element model). Vectors indicate maximum principal strain orientations at the centroid of each element in the FEM in lateral (top) medial (middle) and ventral (bottom) views of the working and balancing sides of the model. Note that the working side in the FEM is the right mandible and the balancing side is the left mandible. Black circles indicate the location of in vivo gage sites from which data was extracted. Strain fields generated in the working side during middle and posterior bites are shown to illustrate the impact of bite point on strain orientations; strain fields showed little variability with change in bite point on the balancing side, thus only middle bites are illustrated. working side. There is no clear trend of increasing or decreasing normal strains for different bite points. In summary, neutral axis orientations on the balancing side, with the exception of one experiment, show low variability across contralateral bite points or experiments.

\section{FEM neutral axis}

Strain normal to the middle posterior section of the Alligator FEM during biting at different locations is illustrated for the working (Fig. 8) and balancing (Fig. 9) sides, and neutral axis orientation and strain magnitudes are listed in Table 14. Unlike the in vivo neutral axis, which is assumed to be straight, the FEM indicates that the neutral axis is more sinuous and its course is influenced by internal structures such as sutures, cavities and tooth roots. Nonetheless, the neutral axis in the FEM passes near the section geometric centroid in nearly all cases.

On the working side, the neutral axis of the Alligator FEM rotates counterclockwise as bite point moves from anterior/middle to posterior (Fig. 8). During anterior biting, the dorsal margin of the mandible is under tension, whereas the ventral border experiences compression, indicating negative bending. During middle bites, compressive strain shifts to the lateral aspect of the mandible and tension occurs on the medial aspect, suggesting the mandible is being bent medially. During middle posterior and posterior bites, the neutral axis is obliquely oriented, with compression occurring dorsally and laterally, and tension located ventrally and medially, indicating positive bending of the mandible with some medial bending.

On the balancing side, the orientation of the neutral axis does not change with changes in contralateral bite point, being obliquely oriented through the corpus. The dorsal margin of the mandible is always in tension, whereas its ventral border is in compression, indicating negative bending. Because compression is shifted slightly to the medial surface (and tension is shifted laterally), there also appears to be some lateral bending of the balancing side. 


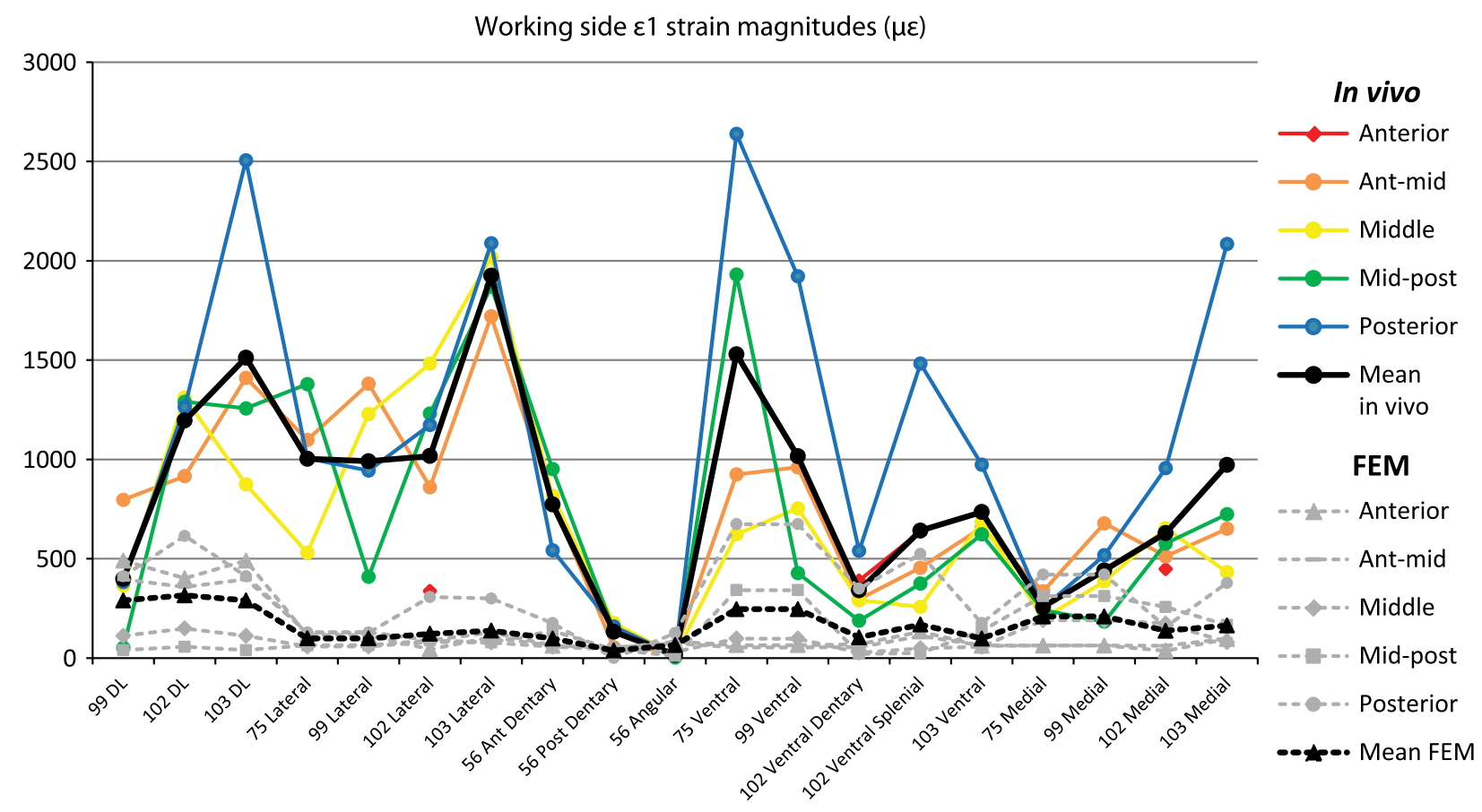

Balancing side $\varepsilon 1$ strain magnitudes $(\mu \varepsilon)$

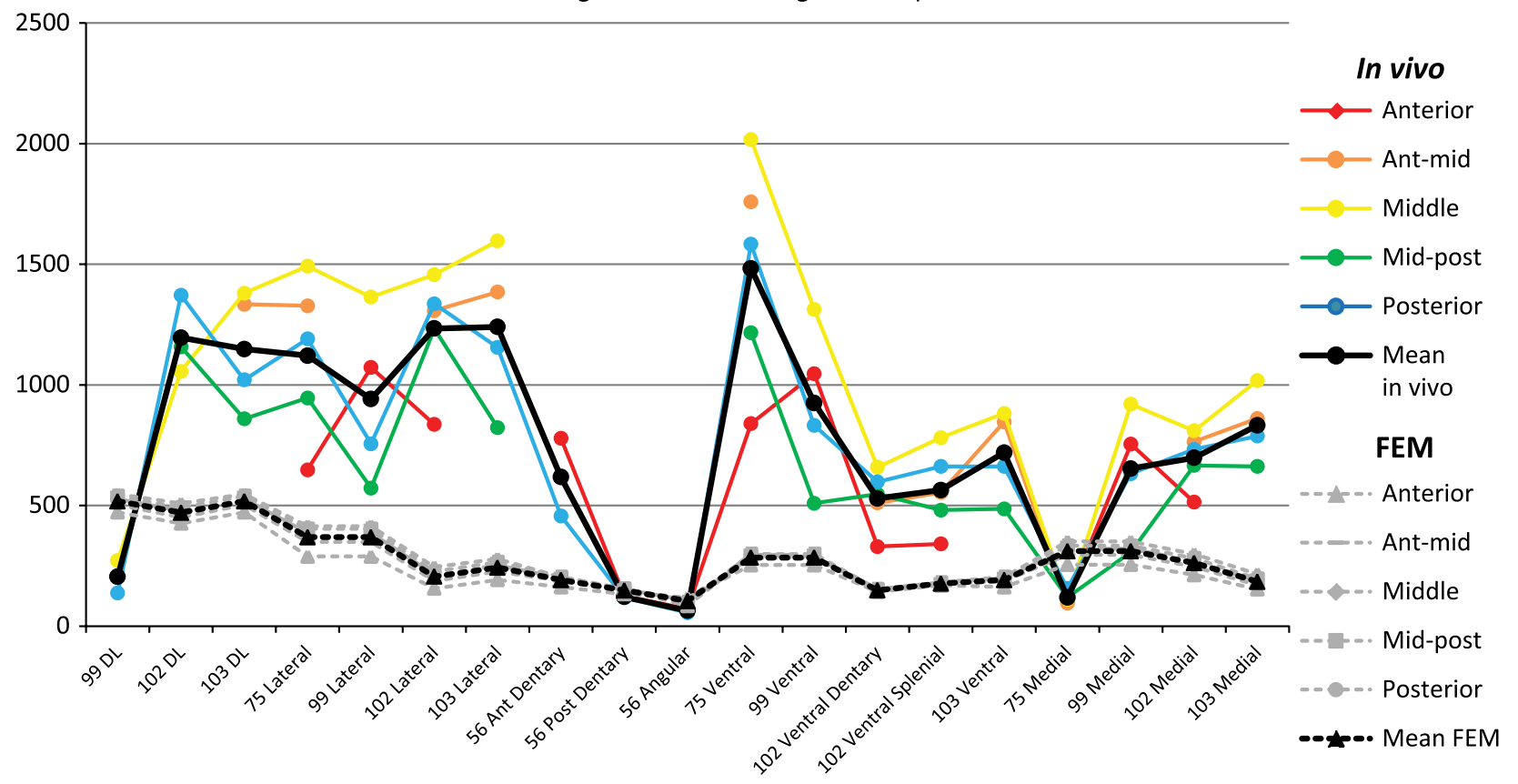

Fig. 5 Mean in vivo and FEM (finite element model) $\varepsilon_{1}$ strain magnitudes. Plots illustrate maximum principal strain at 19 gage sites and their corresponding sites in the FEM. The upper plot shows results from the working side and the lower plot shows results from the balancing side. Both the mean $\varepsilon_{1}$ strains within each gage site and the mean value during biting at different locations are shown. Missing areas of the in vivo plots indicate no data was collected for this bite point at this gage site.

Because of the variability in the orientation of the working side neutral axis observed in vivo, it is difficult to compare neutral axis orientations between the Alligator FEM and in vivo results. In contrast, neutral axis orientations are very similar on the balancing side of the in vivo experiments (except Experiment 99) and the FEM.
Maximum and minimum normal strain magnitudes are lower in the FEM than those calculated in vivo. On the working side, strain magnitudes vary with changes in bite point; on the balancing side, magnitudes are consistent despite changes in bite location. 
Working side $\gamma$ strain magnitudes $(\mu \varepsilon)$

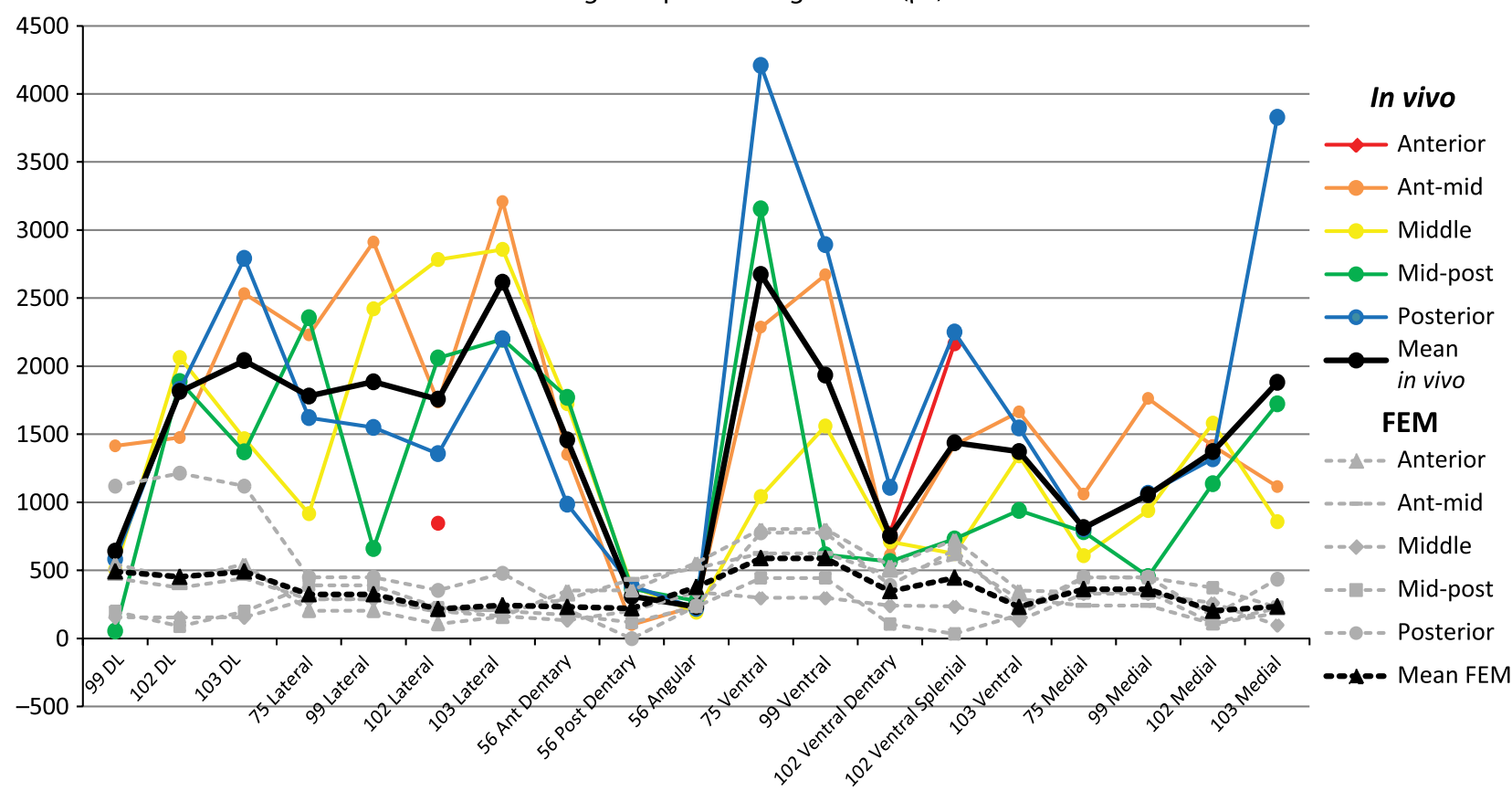

Balancing side $\gamma$ strain magnitudes $(\mu \varepsilon)$

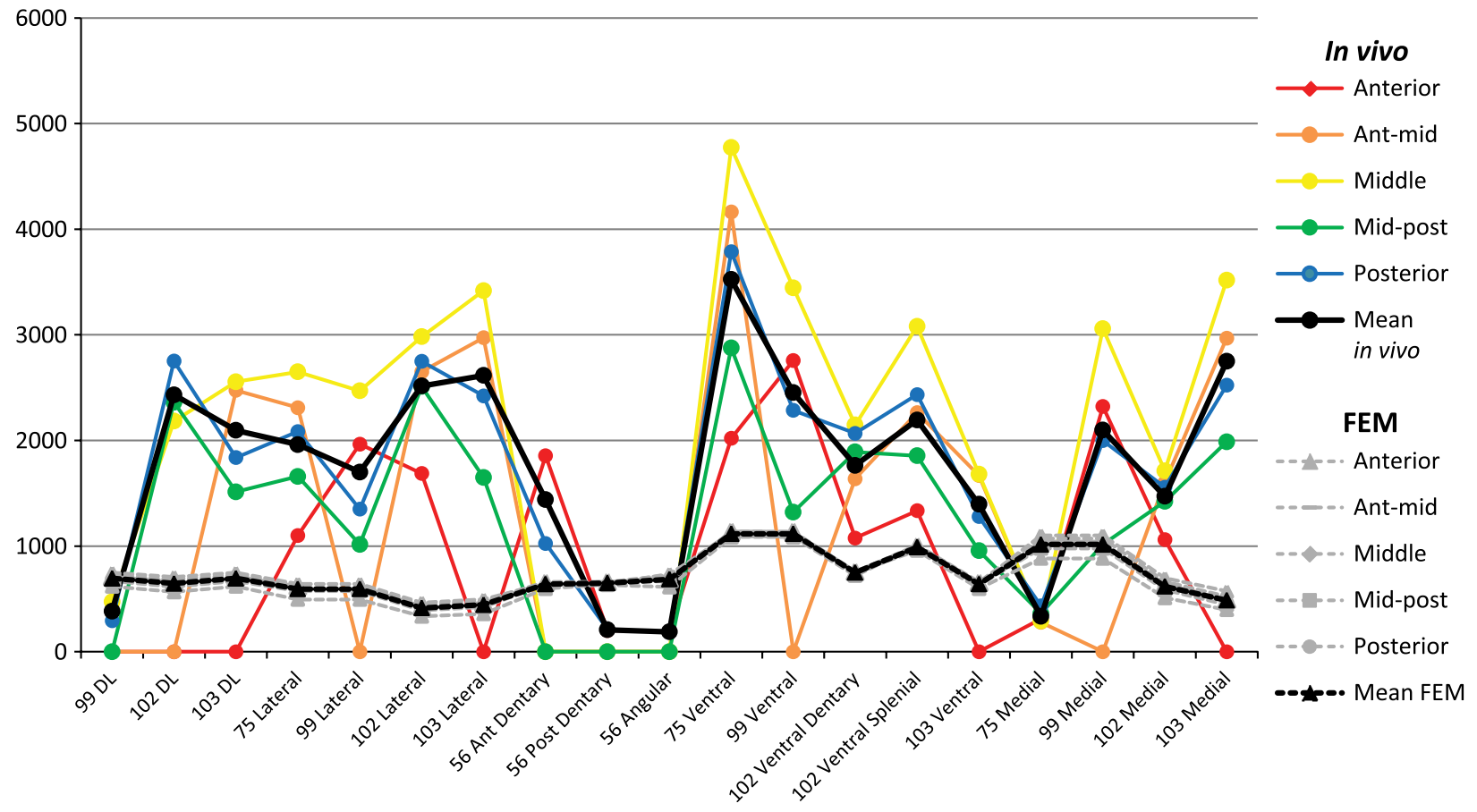

Fig. 6 Plots comparing mean in vivo and FEM (finite element model) shear $(\gamma)$ strain magnitudes. Plots illustrate shear strain at 19 gage sites and their corresponding sites in the FEM. The upper plot shows results from the working side and the lower plot from the balancing side. Both the mean $\gamma$ strains within each gage site and the mean value during biting at different locations are shown.

\section{Discussion}

We have presented new in vivo bone strain data from the Alligator mandible, including strain orientations and magnitudes, and neutral axis of bending; the same data were extracted from the FEM. Here we evaluate our FEM using in vivo data, then use the FEM to understand overall deformation and strain distribution in the mandible during biting. Finally, we compare Alligator strain data and deformation regimes with those known from mammalian mandibles. 
Fig. 7 Contour plots of von Mises strain in the FEM (finite element model). Scale bar indicates strain magnitude (in microstrain) during posterior biting. WS, working side; BS, balancing side in ventral view. For the FEM, the right mandible is always the working side, the left mandible is the balancing side.
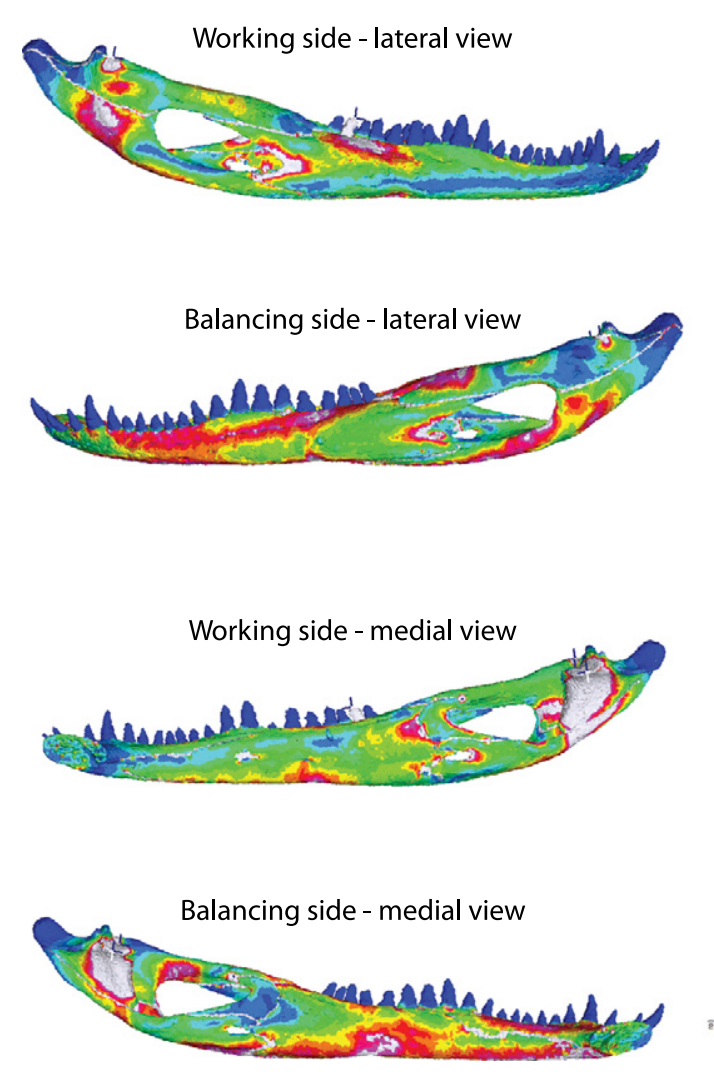

Brick micro strain

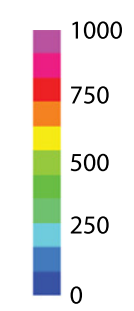

Some problems with the data should be noted. First, due to animal behavior and gage failure, complete datasets are not available for all experiments. Secondly, some experiments gave results that were inconsistent with other experiments; most notable are the results for orientation of the neutral axis in Experiment 99 (Figs 8 and 9) and the principal strain orientations from the lateral gage in Experiment 103 (Figs 2 and 3).

\section{FEM validation and mandibular deformation}

Overall, there is a good correspondence between in vivo and in silico principal strain orientations, including changes in strain orientations with changes in bite point. FEM $\varepsilon_{1}$ strain orientations overlap orientations recorded in vivo at 17 of 19 gage sites during ipsilateral biting and at 10 of 19 sites during contralateral biting. The greatest discrepancy between in vivo and FEM strain orientations occurred on the ventral aspect of the mandible during contralateral biting, during which FEM $\varepsilon_{1}$ strain orientations overlapped in vivo orientations at only three of eight gage sites. It should be noted that strain orientations at three ventral gage sites in Experiment 56, which did not match those obtained from the FEM, also showed poor correspondence with ventral strain orientations obtained in other experiments (Fig. 3). Additionally, strain fields from the ventral aspect of the FEM demonstrate that strain orientations are highly vari- able between gage sites. Thus, although our FEM does not perfectly represent mandibular deformation during biting, general agreement between in vivo and in silico surface strain orientations and the location/orientation of the neutral axis of bending through the corpus suggest our FEM is a reasonable working hypothesis of mandibular deformation in Alligator during biting. The poor correspondence between in vivo and FEM surface strains along the ventral aspect of the mandible may be due these areas being adjacent to sutures, the modeling of which may be imprecise (Reed et al. 2011).

In vivo principal strain orientations and the FEM are used to describe deformation in the mandible during biting. As noted in previous studies (Chalk et al. 2011; Porro et al. 2011; Ross et al. 2011), the FEM itself (its geometry, material properties, and boundary conditions) is our hypothesis of the mechanical behavior of the Alligator mandible; the following descriptors simply summarize the deformation undergone by the mandible during biting.

Analysis of the working side FEM (Fig. 8, and see Porro et al. 2011) suggests that the middle-posterior section of the mandible (where most gages were placed) experiences negative bending (dorsal deformation) during anterior bites, but positive bending (ventral deformation) as the bite point moves posteriorly. Dynamic deformation videos of the FEM (Supporting Information Video S1) illustrate how, during anterior bites, the upward action of the jaw elevator 


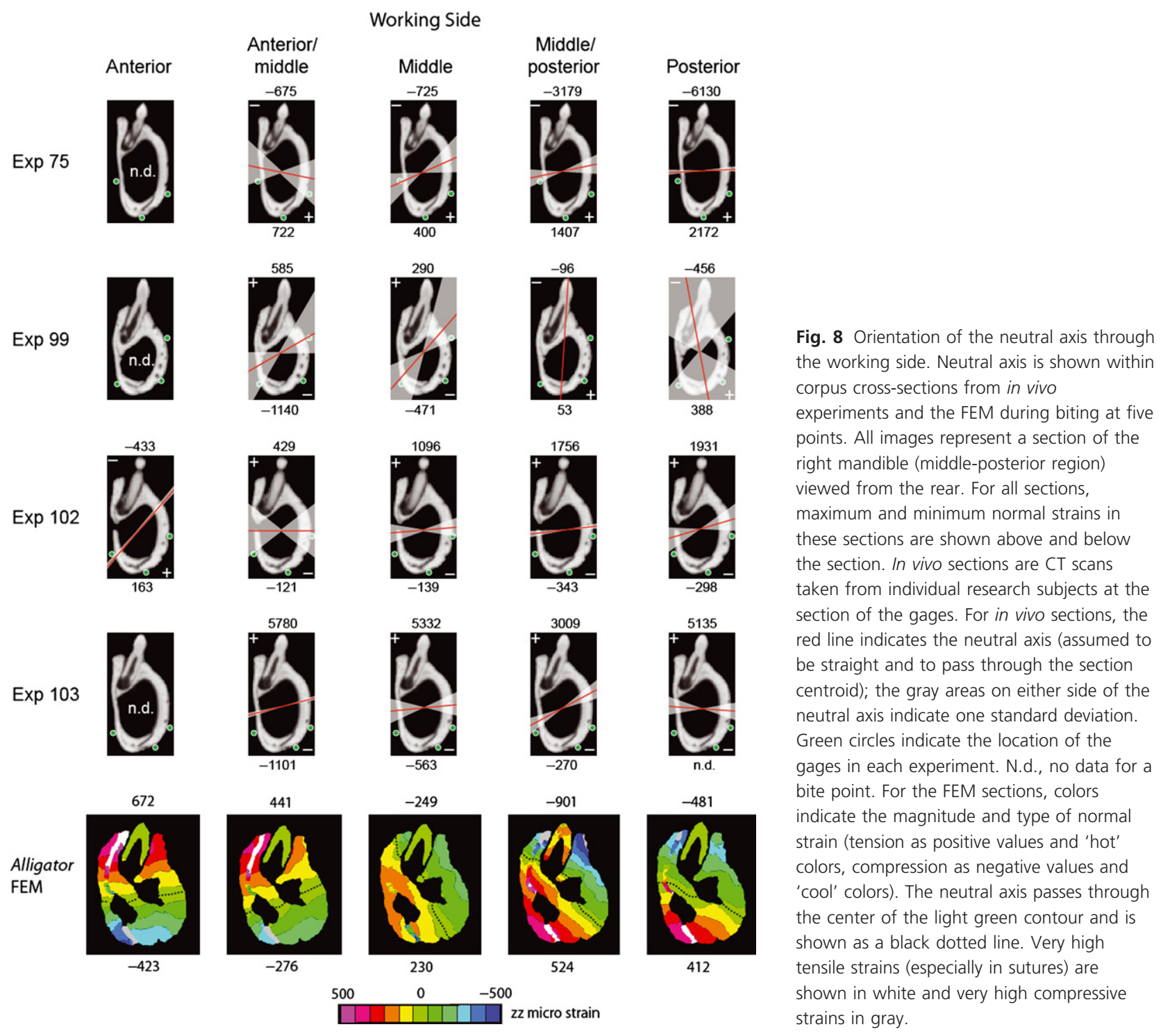

muscles results in the mandible being bent dorsally. During posterior bites, the posterior portion of the mandible experiences dorsal deformation, whereas the portion anterior to the bite point experiences ventral deformation due to forces. At all bite points, the working side mandible is deformed medially, and the ventral margin of the mandible is inverted while the tooth row is everted, indicative of torsion (sensu Figs 9 and 14 in Porro et al. 2011). As in the FEM, during anterior biting in most in vivo experiments $\varepsilon_{1}$ is directed anteroinferiorly on the upper lateral aspect of the working side, rotating clockwise as bite point shifts posteriorly. Strains on the ventral surface of the mandible were directed along the long-axis of the mandible and rotated counterclockwise with a posterior shift in bite point. These strain orientations suggest a combination of negative dorsoventral bending and positive torsion of the working side mandible (eversion of anterior toothrow relative to gaged sections) during anterior biting. As the bite point moves posteriorly, positive bending becomes more important.

Examination of the balancing side FEM (Fig. 9, and see Porro et al. 2011) reveals that, regardless of bite point on the working side mandible, the most important deformation regime on the balancing side is (negative) dorsoventral bending, i.e. the mandible is bent upwards in the middle so that the dorsal edge is in longitudinal tension and the ventral edge is in longitudinal compression. This loading regime is due to the upwardly directed action of the jaw elevators being opposed at the symphysis and jaw joint. The second most important deformation regime in the balancing side mandible is torsion due to inversion of the anterior toothrow relative to the section of the mandible where strain was recorded. Dynamic views of the FEM show inversion of the balancing side tooth row (Supporting Information Videos S1 and S2). In vivo, the lateral orientation of $\varepsilon_{1}$ on the ventral surface of the balancing side mandible is 
Exp 75

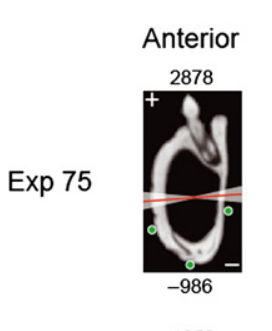

Exp 99

Fig. 9 Orientation of the neutral axis through the balancing side. Neutral axis is shown within corpus cross-sections from in vivo experiments and the FEM during biting at five contralateral points. All images represent a section of the right mandible (middleposterior region) viewed from the rear. For all sections, maximum and minimum normal strains in these sections are shown above and below the section. In vivo sections are CT scans taken from individual research subjects at the section of the gages. For in vivo sections, the red line indicates the neutral axis (assumed to be straight and pass through the section centroid); the gray areas on either side of the neutral axis indicate one standard deviation. Green circles indicate the location of the gages in each experiment. N.d., no data for a bite point. For the FEM sections, colors indicate the magnitude and type of normal strains (tension as positive values and 'hot' colors, compression as negative values and 'cool' colors). The neutral axis passes through the center of the light green contour and is shown as a dotted line. Very high tensile strains are shown in white and very high compressive strains in gray. (1)
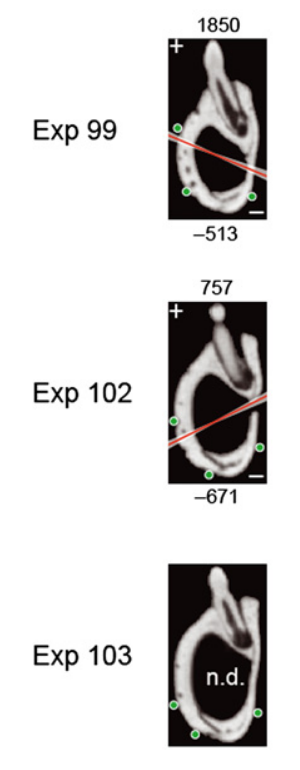
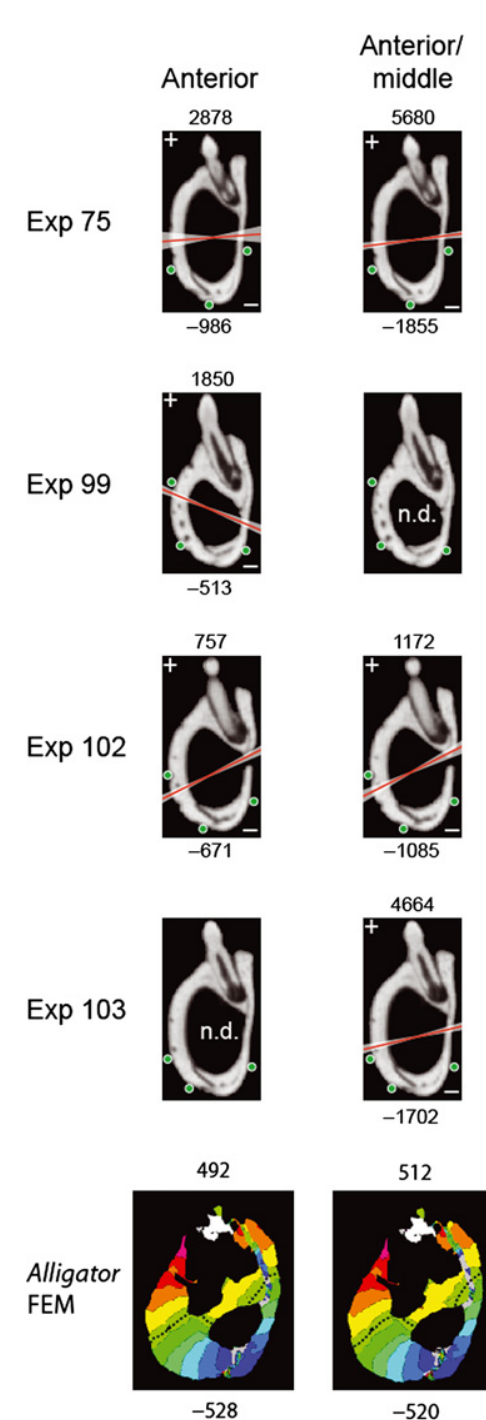

Balancing Side

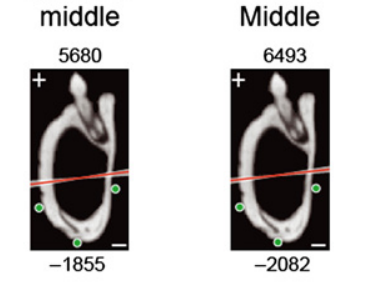

Middle/
posterior
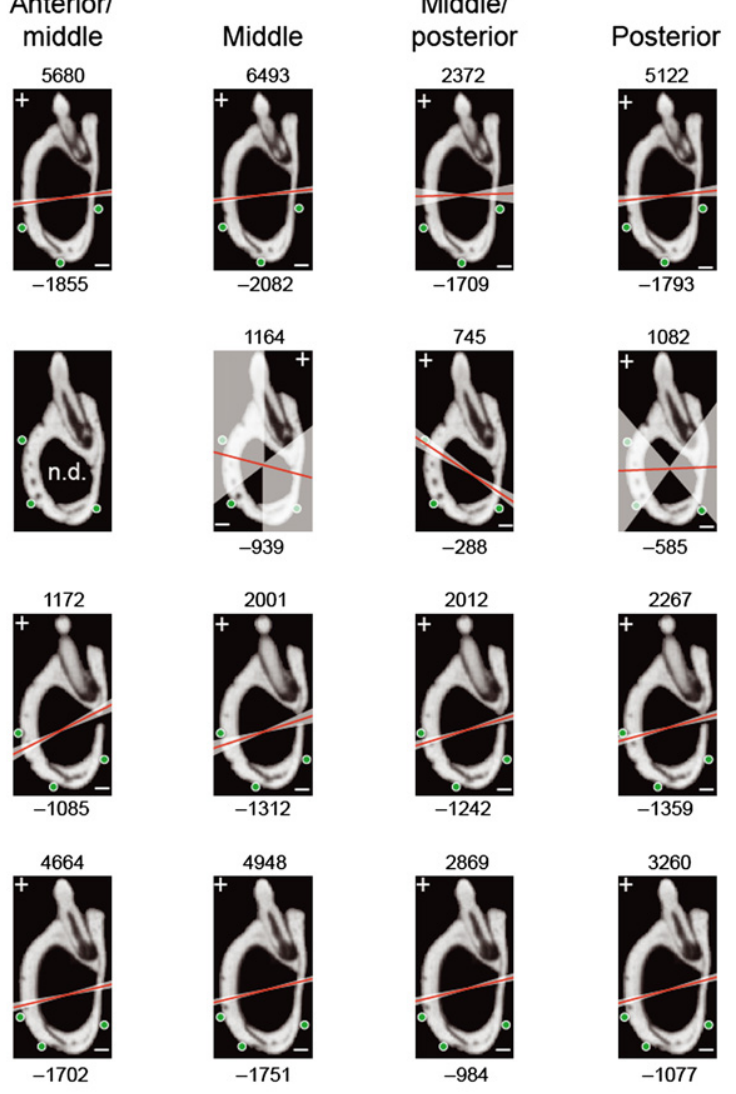

512

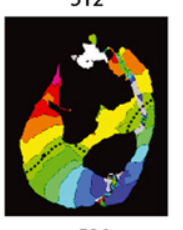

501

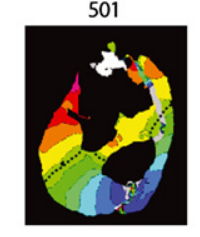

417

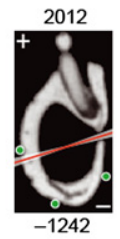

479
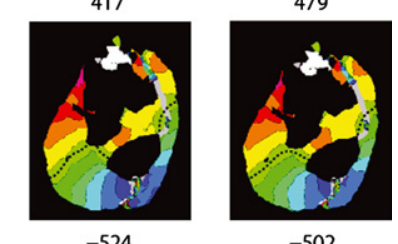

$-502$

$-500$

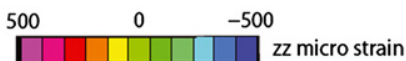

indicative of negative dorsoventral bending regime. Moreover, the anterosuperiorly directed components of $\varepsilon_{1}$ on the lateral aspect of the mandible in vivo and the anteroinferiorly directed components on the medial aspect are suggestive of the torsional regime described above. As in the $\mathrm{FEM}$, in vivo balancing side strain orientations change little with changes in bite location. Cross-sections through both the in vivo mandible and FEM (Fig. 9) corroborate predictions from strain orientations, demonstrating that the mandible is being bent dorsally (negative bending) at all bite points, although slight counterclockwise rotation of the neutral axis suggests some lateral bending.

For all gage sites in all experiments, $\varepsilon_{1}$ orientation during ipsilateral biting is both different from and more variable (with changes in bite point) than during contralateral bites 13 of the 19 gages exhibited statistically significant differences in $\varepsilon_{1}$ orientations $(P<0.05)$ for different bite points during ipsilateral bites; only eight of 19 gage sites exhibited significant differences in $\varepsilon_{1}$ orientations with changes in bite point during contralateral bites (Table 13). The orientation of the neutral axis is also more variable on the working side than on the balancing side. Thus, both in vivo and in silico results clearly demonstrate the impact of bite force and bite location on the mechanical behavior of the working side. Furthermore, inter-experimental variation in $\varepsilon_{1}$ orientation is greater on the working side than on the balancing side. Lastly, on the working side, variation in $\varepsilon_{1}$ orientation is lowest on the lateral aspect of the mandible (mean $r=0.83$ ), and highest on the medial aspect (mean $r=0.49$ ); whereas on the balancing side, variation in $\varepsilon_{1}$ orientation was lowest on the medial aspect of the mandible (mean $r=0.97$ ).

Circular-linear analyses reveal correlations between strain orientation and magnitude at some gage sites. These correlations are not unexpected as there can only be one load- 
222 Alligator mandibular bone strain, L. B. Porro et al.

Table 14 Descriptive statistics for orientation of neutral axis of bending $(\alpha)$ and calculated maximum and minimum normal strains for all experiments and the FEM.

\begin{tabular}{|c|c|c|c|c|c|c|c|c|c|}
\hline \multirow[b]{2}{*}{ Experiment } & \multirow[b]{2}{*}{ Bite side } & \multirow{2}{*}{$\begin{array}{c}\text { Bite } \\
\text { position* }\end{array}$} & \multirow[b]{2}{*}{$n$} & \multicolumn{2}{|c|}{$\begin{array}{l}\text { Neutral axis } \\
\text { orientation }(\alpha)\end{array}$} & \multicolumn{2}{|c|}{$\begin{array}{c}\text { Maximum } \\
\text { calculated normal } \\
\text { strain }(\mu \varepsilon)\end{array}$} & \multicolumn{2}{|c|}{$\begin{array}{c}\text { Minimum } \\
\text { calculated normal } \\
\text { strain }(\mu \varepsilon)\end{array}$} \\
\hline & & & & Mean & SD & Mean & SD & Mean & SD \\
\hline \multirow[t]{12}{*}{75} & Left (WS) & $\mathrm{a} / \mathrm{m}$ & 4 & 11.6 & 32.4 & 722 & 580 & -675 & 381 \\
\hline & & $\mathrm{m}$ & 6 & -24.1 & 23 & 400 & 246 & -725 & 822 \\
\hline & & $\mathrm{m} / \mathrm{p}$ & 12 & -11.8 & 15.1 & 1407 & 888 & -3179 & 2729 \\
\hline & & $\mathrm{p}$ & 8 & -2.8 & 2.7 & 2172 & 844 & -6130 & 2463 \\
\hline & All left & & 30 & -8.8 & 20.2 & 1318 & 972 & -3142 & 2989 \\
\hline & Right (BS) & $a$ & 4 & 4.3 & 8.5 & 2878 & 659 & -986 & 198 \\
\hline & & $\mathrm{a} / \mathrm{m}$ & 3 & 7.4 & 0.9 & 5680 & 931 & -1855 & 271 \\
\hline & & $\mathrm{m}$ & 19 & 6.5 & 1.2 & 6493 & 1518 & -2082 & 470 \\
\hline & & $\mathrm{m} / \mathrm{p}$ & 8 & 1.8 & 9.7 & 2373 & 2018 & -1709 & 1414 \\
\hline & & $p$ & 5 & 6.2 & 4.6 & 5122 & 2375 & -1793 & 670 \\
\hline & All right & & 39 & 5.5 & 5.3 & 5056 & 2367 & -1829 & 774 \\
\hline & All bites & & 69 & 0.1 & 14.8 & 3637 & 2671 & -2328 & 2025 \\
\hline \multirow[t]{11}{*}{99} & Right (WS) & $\mathrm{a} / \mathrm{m}$ & 2 & 30 & 30.3 & 585 & 186 & -1140 & 676 \\
\hline & & $\mathrm{m}$ & 9 & 48.8 & 26.1 & 290 & 188 & -471 & 312 \\
\hline & & $\mathrm{m} / \mathrm{p}$ & 1 & 86.3 & & 53 & & -96 & \\
\hline & & $p$ & 9 & 100.5 & 50.9 & 388 & 193 & -456 & 475 \\
\hline & All right & & 21 & 69.6 & 46 & 346 & 206 & -508 & 444 \\
\hline & Left (BS) & $a$ & 2 & 22.6 & 1.7 & 1850 & 431 & -513 & 126 \\
\hline & & $\mathrm{m}$ & 3 & -37.6 & 52.3 & 1164 & 337 & -939 & 535 \\
\hline & & $\mathrm{m} / \mathrm{p}$ & 7 & 33.5 & 3.4 & 745 & 310 & -288 & 71 \\
\hline & & $p$ & 3 & -2.4 & 51.5 & 1082 & 725 & -575 & 219 \\
\hline & All left & & 15 & 10.6 & 39.9 & 1044 & 535 & -506 & 340 \\
\hline & All bites & & 36 & 45.9 & 52.1 & 629 & 507 & -507 & 400 \\
\hline \multirow[t]{13}{*}{102} & Left (WS) & $a$ & 2 & -49.1 & 1.8 & 163 & 8 & -433 & 149 \\
\hline & & $\mathrm{a} / \mathrm{m}$ & 9 & 0.1 & 38.3 & 429 & 402 & -121 & 183 \\
\hline & & $\mathrm{m}$ & 19 & -5 & 14.4 & 1096 & 561 & -139 & 167 \\
\hline & & $\mathrm{m} / \mathrm{p}$ & 6 & -6.9 & 4.3 & 1756 & 1232 & -343 & 319 \\
\hline & & $p$ & 9 & -16.6 & 19.1 & 1931 & 803 & -298 & 384 \\
\hline & All left & & 45 & -8.5 & 23 & 189 & 880 & -212 & 258 \\
\hline & Right (BS) & $a$ & 2 & 26.5 & 2.4 & 757 & 36 & -671 & 46 \\
\hline & & $a / m$ & 2 & 26.8 & 4.7 & 1172 & 439 & -1055 & 281 \\
\hline & & $\mathrm{m}$ & 9 & 18.1 & 6.9 & 2001 & 695 & -1312 & 440 \\
\hline & & $\mathrm{m} / \mathrm{p}$ & 11 & 17.1 & 2.6 & 2012 & 927 & -1242 & 547 \\
\hline & & $p$ & 12 & 15.3 & 3.4 & 2267 & 1146 & -1359 & 654 \\
\hline & All right & & 36 & 17.8 & 5.4 & 1978 & 962 & -1256 & 541 \\
\hline & All bites & & 81 & 3.2 & 22 & 1532 & 986 & -674 & 653 \\
\hline \multirow[t]{11}{*}{103} & Right (WS) & $a / m$ & 9 & 14.5 & 3 & 5780 & 1010 & -1101 & 436 \\
\hline & & $\mathrm{m}$ & 3 & 5.6 & 19.7 & 5332 & 2985 & -563 & 47 \\
\hline & & $\mathrm{m} / \mathrm{p}$ & 4 & 28.3 & 11.2 & 3009 & 1425 & -270 & \\
\hline & & $p$ & 8 & -4.1 & 14.2 & 5135 & 1501 & & \\
\hline & All right & & 24 & 9.5 & 15.9 & 5047 & 1739 & -645 & 241 \\
\hline & Left (BS) & $\mathrm{a} / \mathrm{m}$ & 6 & 13.3 & 3.1 & 4664 & 1063 & -1702 & 355 \\
\hline & & $\mathrm{m}$ & 3 & 13.6 & 0.4 & 4948 & 945 & -1751 & 282 \\
\hline & & $\mathrm{m} / \mathrm{p}$ & 6 & 12.1 & 4.4 & 2869 & 1295 & -984 & 498 \\
\hline & & $\mathrm{p}$ & 4 & 14.9 & 5 & 3260 & 1193 & -1077 & 438 \\
\hline & All left & & 19 & 13.6 & 3.7 & 3846 & 1392 & -1351 & 524 \\
\hline & All bites & & 43 & -43.5 & 45.7 & 3137 & 1486 & -2191 & 1447 \\
\hline
\end{tabular}


Table 14. (continued)

\begin{tabular}{|c|c|c|c|c|c|c|c|c|c|}
\hline \multirow[b]{2}{*}{ Experiment } & \multirow[b]{2}{*}{ Bite side } & \multirow{2}{*}{$\begin{array}{c}\text { Bite } \\
\text { position* }\end{array}$} & \multirow[b]{2}{*}{$n$} & \multicolumn{2}{|c|}{$\begin{array}{l}\text { Neutral axis } \\
\text { orientation }(\alpha)\end{array}$} & \multicolumn{2}{|c|}{$\begin{array}{c}\text { Maximum } \\
\text { calculated normal } \\
\text { strain }(\mu \varepsilon)\end{array}$} & \multicolumn{2}{|c|}{$\begin{array}{c}\text { Minimum } \\
\text { calculated normal } \\
\text { strain }(\mu \varepsilon)\end{array}$} \\
\hline & & & & Mean & SD & Mean & SD & Mean & SD \\
\hline \multirow[t]{13}{*}{ FEM } & Right (WS) & a & & 7 & & 672 & & -423 & \\
\hline & & $\mathrm{a} / \mathrm{m}$ & & 21 & & 441 & & -276 & \\
\hline & & $\mathrm{m}$ & & -62 & & 230 & & -249 & \\
\hline & & $\mathrm{m} / \mathrm{p}$ & & -43 & & 524 & & -901 & \\
\hline & & $\mathrm{p}$ & & -23 & & 412 & & -381 & \\
\hline & All left & & & -20 & & 456 & & -446 & \\
\hline & Left (BS) & a & & 28 & & 492 & & -528 & \\
\hline & & $\mathrm{a} / \mathrm{m}$ & & 31 & & 512 & & -520 & \\
\hline & & $\mathrm{m}$ & & 31 & & 501 & & -524 & \\
\hline & & $\mathrm{m} / \mathrm{p}$ & & 31 & & 417 & & -524 & \\
\hline & & $p$ & & 30 & & 479 & & -502 & \\
\hline & All right & & & 30 & & 480 & & -520 & \\
\hline & All bites & & & 5 & & 468 & & -483 & \\
\hline
\end{tabular}

a, anterior; a/m, anterior/middle; $\mathrm{m}$, middle; $\mathrm{m} / \mathrm{p}$, middle/posterior; $\mathrm{p}$. posterior.

ing/stress/strain regime during maximal contraction of the jaw elevator muscles. As muscle recruitment approaches this value, there are likely to be fewer ways of generating bite force.

\section{Strain magnitudes and gradients}

Absolute principal and shear strain magnitudes were substantially lower in our FEM than data recorded at nearly all corresponding gage sites in vivo. Good correspondence in principal strain orientation with large discrepancies in principal strain magnitude is common in validation studies of vertebrate skulls, being noted when comparing in vivo and FEM results in the crania of primates (Ross et al. 2005, 2011; Kupczik et al. 2009) and crocodilians (Metzger et al. 2005). Better correspondence has been found between FEM and in vitro strain magnitudes in validation studies of the macaque mandible (Kupczik et al. 2007; Panagiotopoulou et al. 2010, 2011), ostrich mandible (Rayfield, 2011) and pig cranium (Bright \& Rayfield, 2011). However, it should be noted that in some of these studies material properties were changed and models made less stiff before comparable strain magnitudes were obtained in post hoc analyses.

Various factors may be responsible for the discrepancy in strain magnitudes between the in vivo data and the FEM. We used a value of $300 \mathrm{kN} \mathrm{m}^{-2}$ for the specific tension of muscle, a value commonly found in the literature (Sinclair \& Alexander, 1987); nonetheless, it is possible that Alligator jaw muscle contracts with greater force. Recent multi-body dynamics analyses of the diapsid Sphenodon have suggested that the specific tension of jaw muscles in this taxon must be significantly higher $\left(890 \mathrm{kN} \mathrm{m}^{-2}\right)$ to generate bite forces recorded in vivo (Curtis et al. 2010); furthermore, FEA of the skulls of multiple crocodilian species using a specific muscle tension of $300 \mathrm{kN} \mathrm{m}^{-2}$ resulted in bite forces consistently and significantly lower than those reported in vivo (Erickson et al. 2012; Walmsley et al. 2013). Our model incorporated recently reported material properties for Alligator mandibular cortical bone (Zapata et al. 2010); however, there are no data on the material properties of reptilian cranial sutures. It is possible that the Young's modulus assigned to the sutures within our model is too elastic, allowing the sutures to act as 'strain sinks' that decrease strain in surrounding bone (Buckland-Wright, 1978; Rafferty et al. 2003). Moreover, our model does not incorporate less stiff trabecular bone in the articular of the Alligator mandible, possibly rendering the model too stiff. Lastly, constraints have a tremendous impact on strain magnitudes within FEMs (Marinescu et al. 2005). We constrained the mandible at three nodes aligned mediolaterally across each articular, as well as a single node on the medial aspect of each mandible. This combination of constraints was found to produce the closest correspondence to in vivo data in terms of strain orientations. However, it is possible that our model is over-constrained, resulting in low strain magnitudes. Ongoing sensitivity analyses will shed further light on the input parameters impacting model behavior.

Strain gradients as visualized in FEA contour plots reveal very high strains at the articular and surrounding the external mandibular fenestra, suggesting that morphology in these regions may be better optimized for resisting forces exerted by the jaw adductors and joint reaction forces than those related to bite force. Interestingly, the working side mandibular corpus exhibits low 
strains (except immediately below the bite point) while the ventral aspect of the balancing side and the symphysis are highly strained. This concurs with in vivo and FEM strain data that demonstrate that the balancing side mandible experiences higher principal and shear strains than the working side.

\section{Form-function relationships in the Alligator mandible}

Our in vivo experiments and FE modeling only addressed the mechanical behavior of the mandible during biting; we did not elicit or attempt to model other feeding behaviors such as prey shaking and body rolling. Nevertheless, some comments regarding form-function relationships in the Alligator mandible are possible.

Van Drongelen \& Dullemeijer's (1982) analysis of mandible morphology in Caiman assumed that resistance to bending stress played a significant role in crocodilian mandible design. Although the in vivo and FEM results presented here and in Porro et al. (2011) confirm this assumption, they also suggest that other loading regimes are important, such as torsion and mediolateral bending. Moreover, (Porro et al. 2011) demonstrated that mandibular geometry interacts with bone and suture material properties to improve resistance of the mandible to external forces. A complete optimization analysis of Alligator mandible form should take these into account.

How does the Alligator mandible withstand the high strains recorded in vivo and estimated within cross-sections (Figs 8 and 9)? The strains recorded here are the largest reported from any vertebrate mandible: $>4000 \mu \varepsilon$ in tension in the biting side ventral dentary in Experiment 75 (Table 3), and $<-3400 \mu \varepsilon$ in compression from the same gage on the working side. These values exceed those reported from the limb bones of galloping mammals (Biewener \& Taylor, 1986), which are generated when the body mass is supported on one limb, whereas the mandibular strains recorded in our experiments were generated by the jaw elevator muscles alone. Moreover, if our calculations of normal strains in mandibular cross-sections are correct, maximum normal strains in the mandible exceed 5000 or even $6000 \mu \varepsilon$ in tension (Figs 8 and 9). Given that vertebrate bone yields at around 4500-9400 $\mu \varepsilon$ under static loading (Currey, 1990), and at even lower magnitudes under repetitive loading conditions, it seems unlikely that Alligator mandibles experience these strains regularly. Nevertheless, we conjecture that the defensive bites elicited in experimental settings may have resulted in close to the maximum stress and strains experienced by the Alligator mandible.

\section{Comparison of mandible deformation in Alligator and mammals}

The only other vertebrates for which extensive in vivo mandibular bone strain data are available are primate and ungulate mammals. The alligator feeding system differs from that of mammals both structurally (previously discussed) and in various functional aspects, including lack of translation at the craniomandibular joint, the magnitude of the bite forces experienced by the mandible, and the much lower number of daily loading events. These differences make it difficult to isolate reasons for differences in deformation and strain regimes. Nevertheless, some interesting observations are possible.

Free body analysis and in vivo bone strains suggest that during mastication and biting, the working side mandible of primates is twisted about its long axis, with the tooth row and mandibular angle being everted. Additionally, the corpus is sheared perpendicular to its long axis and bent in sagittal planes; however, these shear and bending stresses are less important than torsion on the working side. The balancing side primate mandible is bent during mastication, but both bent and twisted during isometric biting (Hylander, 1977, 1979a,b, 1981, 1984).

In Alligator, the deformation regime of the balancing side mandible closely resembles that of anthropoid primates, i.e. strong negative bending. In contrast, torsion is much less important than dorsoventral bending in the working side Alligator mandible (Porro et al. 2011), a difference attributable to the relatively longer mandible of Alligator. The most significant differences between primate and alligator mandible deformation regimes are in the region of the jaw muscle attachments. In primates the laterally directed masseter muscle everts the angle of the mandible (Hylander, 1979b). In contrast, the angle of the Alligator mandible is inverted; furthermore, the Alligator mandible experiences relatively large mediolateral bending stresses due to the orientation of the jaw elevator muscles, which generate large reaction forces at the jaw joint and pterygoid flange (Porro et al. 2011). The significance of this difference in loading regime for mandibular design in mammalian and non-mammalian vertebrates has yet to be evaluated.

Primates (Hylander et al. 1987), rabbits (Weijs \& De Jongh, 1977) and hyraxes (Lieberman et al. 2004) experience different loading regimes on the working and balancing side mandibles; pigs are the only mammal studied to date that show similar loading regimes on the working and balancing sides, which may be related to their isognathous chewing and bilateral mastication (Herring \& Scapino, 1973; Herring et al. 2001). Although mammals for which electromyographic data are available show differences in the magnitude and timing of activation between working and balancing jaw elevator muscles, crocodilians are thought to use their jaw elevators synchronously and symmetrically (Van Drongelen \& Dullemeijer, 1982; Busbey, 1989; Cleuren \& De Vree, 1995). This suggests that differences in stress and strain regimes on working and balancing side in Alligator are due to differences in reaction forces. 


\section{Concluding remarks}

The results presented here document for the first time in vivo bone strain in a sauropsid mandible during feeding. The deformation regime (inferred from principal strain orientations and neutral axis orientations) and strain magnitudes described here will serve as a baseline with which the mandibles of other sauropsids can be compared. Such future comparative studies, encompassing crocodilians, extinct archosaurs, squamates and turtles, may highlight similarities or differences in the mechanical response of the mandibles due to diet, feeding behavior and musculature, reflected in either convergent or disparate mandibular morphology.

As part of this study, we compared strain magnitudes and orientations (as well as neutral axes of bending) collected in vivo to data produced by a high-resolution FEM. Although this model has been the basis of previous studies, the accuracy of its predictions awaited validation against in vivo data. The results presented here suggest that our FEM of the Alligator mandible accurately predicts principal strain and neutral axis orientations (and, by extension, deformation regime) during biting at different points along the tooth row. Although FEA results should be validated against in vivolin vitro data when feasible, this is not possible for extinct forms. Our results suggest that given accurate geometry, material properties and boundary conditions, FEMs of sauropsid mandibles can be used to model deformation regimes realistically, highlighting the power of FEA in a comparative context.

In contrast, principal and shear strain magnitudes exhibited by the FEM are substantially lower than those recorded in vivo. Potential reasons for this discrepancy have previously been discussed; however, until the underlying cause is identified, we would caution researchers about using absolute values obtained from skull FEMs - for example, to predict maximum bite forces or skull safety factors. Furthermore, our FEM/in vivo comparison suggests that areas immediately adjacent to the application of loads (jaw joint and bite point) and areas immediately adjacent to sutures experience highly variable strain orientations; results from these areas should be thus be interpreted cautiously.

Both in vivo experimental data and the FEM highlight differences in the mechanical behavior of the Alligator mandible compared with that of primate mandibles. Although the primary deformation regime of the primate mandible, particularly the working side, is torsion about its long axis, the mandible of Alligator experiences a greater degree of dorsoventral bending, due to its relatively greater length. Furthermore, the medial placement of the jaw elevator muscles in diapsids (compared with their medial and lateral placement in mammals) reverses the direction of torsion experienced by the posterior portion of the Alligator mandible compared with that of primates. Strain magnitudes recorded in the Alligator mandible are much larger than those reported in mammals, and higher even than peak principal strains recorded in the crania of the lizards Uromastyx and Tupinambis (C. F. Ross, A. Herrel, L. B. Porro, K. Murray, S. Evans and M. Fagan, in review; L.B. Porro, C.F. Ross., J. Iriarte-Diaz, J. O'Reilly, S.E. Evans and M.J. Fagan, unpublished data). Strong strain gradients within the mandible, as illustrated by the FEM, suggest that certain regions of the mandible may be more strongly adapted to resist feeding forces (either reaction forces at the jaw joint or bite point or forces exerted by muscles) than other areas.

Both the in vivo and FEM data highlight the impact of bite point on the mechanical response of the mandible. Ongoing sensitivity analyses are evaluating the effect of other input variables, including material properties, constraints and muscle force, on model behavior. The availability of in vivo data will allow us to determine not only how these input parameters impact behavior, but also which specific combination produce the most accurate results.

\section{Acknowledgements}

The animal care and veterinary staff at Stony Brook University provided expert care of our animals. B. Demes kindly provided code in IGOR Pro 4.0. Justin Lemberg (University of Chicago) segmented CT scan data of Alligator. Christian Wietholt (Visage Imaging, Inc.) provided assistance with AMIRA and Anne Delvaux (Beauort Analysis) provided technical support for STRAND7. Casey Holliday (University of Missouri) provided his expertise during muscle mapping of the FEM. Analysis of Alligator jaw muscle architecture was carried out by Fred Anapol (University of Wisconsin).

\section{Author contributions}

All authors conceived of and designed the research. K.A.M. and C.F.R. collected in vivo data. K.A.M., C.F.R. and L.B.P. processed and interpreted in vivo data. L.B.P. built, loaded and interpreted the FEM. J.I.D. and C.F.R. wrote the MATLAB code for extracting FEM data. J.I.D. extracted and processed surface strain data from the FEM. L.B.P., K.A.M. and C.F.R. drafted the manuscript. All authors read and commented on the manuscript.

\section{References}

Bell PR, Snively E, Shychoski L (2009) A comparison of the jaw mechanics in hadrosaurid and ceratopsid dinosaurs using finite element analysis. Anat Rec (Hoboken) 292, 1338-1351.

Berens P (2009) CircStat: a MATLAB toolbox for circular statistics. J Stat Softw 31, p. 21. Available at http://www.jstatsoft.org/ v31/i10.

Biewener AA, Taylor CR (1986) Bone strain: a determinant of gait and speed? J Exp Bio/ 123, 383-400.

Bock WJ (1966) An approach to the functional analysis of bill shape. Auk 83, 10-51.

Bock WJ, Kummer B (1968) The avian mandible as a structural girder. J Biomech 1, 89-96. 
Bock WJ, Von Wahlert G (1965) Adaptation and the formfunction complex. Evolution 19, 269-299.

Bright JA, Rayfield EJ (2011) Sensitivity and ex vivo validation of finite element models of the domestic pig cranium. J Anat 219, 456-471.

Buckland-Wright JC (1978) Bone structure and the patterns of force transmission in the in the cat skull (Felis cattus). $J$ Morphol 155, 35-62.

Busbey $A B$ (1989) Form and function of the feedng apparatus of Alligator mississippiensis. J Morphol 202, 99-127.

Busbey AB (1995) The structural consequences of skull flattening in crocodilians. In: Functional Morphology in Vertebrate Paleontology. (ed. Thomason JJ), pp. 173-192, Cambridge: Cambridge University Press.

Carter DR, Harris WH, Vasu R, et al. (1981) The mechanical and biological response of cortical bone to in vivo strain histories. In: Mechanical Properties of Bone (AMD vol. 45). (ed. Cowin SC), pp. 81-92, New York: American Society of Mechanical Engineers.

Chalk J, Richmond BG, Ross CF, et al. (2011) A finite element analysis of masticatory stress hypotheses. Am J Phys Anthropol 145, 1-10.

Cleuren J, Aerts P, De Vree F (1995) Bite and joint force analysis in Caiman crocodilus. Belg J Zool 125, 79-94.

Currey JD (1990) Physical characteristics affecting the tensile failure properties of compact bone. J Biomech 23, 837-844.

Currey JD (2002) Bone: Structure and Mechanics. Princeton: Princeton University Press.

Curtis N, Jones MEH, Lappin AK, et al. (2010) Comparison between in vivo and theoretical bite performance: using multi-body modelling to predict muscle and bite forces in a reptile skull. J Biomech 43, 2804-2809.

Daniel WJT, McHenry C (2001) Bite force to skull stress correlation-modelling the skull of Alligator mississippiensis. In: Crocodilian Biology and Evolution. (eds Grigg GC, Seebacher F, Franklin CE), pp. 135-143, Surrey Beatty and Sons: Chipping Norton.

Demes B (2007) In vivo bone strain and bone functional adaptation. Am J Phys Anthropol 133, 717-722.

Demes B, Qin YX, Stern JT, et al. (2001) Patterns of strain in the macaque tibia during functional activity. Am J Phys Anthropol 116, 257-265.

Erickson GM, Lappin AK, Vliet KA (2003) The ontogeny of biteforce performance in American alligator (Alligator mississippiensis). J Zool 260, 317-327

Erickson GM, Gignac PM, Steppan SJ, et al. (2012) Insights into the ecology and evolutionary success of crocodilians revealed through bite-force and tooth-pressure experimentation. PLOS ONE 7, e31781.

Grosse IR, Dumont ER, Coletta C, et al. (2007) Techniques for modeling muscle-induced forces in finite element models of skeletal structures. Anat Rec (Hoboken) 290, 1069-1088.

Herring SW, Scapino R (1973) Physiology of feeding in miniature pigs. J Morphol 141, 427-460.

Herring SW, Rafferty KL, Liu ZJ, et al. (2001) Jaw muscles and the skull in mammals: the biomechanics of mastication. Comp Biochem Physiol Part A 131, 207-219.

Hibbeler RC (2000) Mechanics of Materials. Upper Saddle River, NJ: Prentice Hall.

Holliday CM, Witmer LM (2007) Archosaur adductor chamber evolution: integration of musculoskeletal and topological criteria in jaw muscle homology. J Morphol 268, 457-484.
Hylander WL (1977) In vivo bone strain in the mandible of Galago crassicaudatus. Am J Phys Anthropol 160, 223-240.

Hylander WL (1979a) The functional significance of primate mandibular form. J Morphol 160, 223-240.

Hylander WL (1979b) Mandibular function in Galago crassicaudatus and Macaca fascicularis: an in vivo approach to stress analysis of the mandible. J Morphol 159, 253-296.

Hylander WL (1981) Patterns of stress and strain in the macaque mandible. In: Craniofacial Biology. Monograph No. 10, Craniofacial Growth Series. (ed. Carlson DS), pp. 1-35, Ann Arbor, MI: Center for Human Growth and Development.

Hylander WL (1984) Stress and strain in the mandibular symphysis of primates: a test of competing hypotheses. Am J Phys Anthropol 64, 1-46.

Hylander WL, Crompton AW (1986) Jaw movements and patterns of mandibular bone strain during mastication in the monkey Macaca fasicularis. Arch Oral Biol 31, 841-848.

Hylander WL, Johnson KR, Crompton AW (1987) Loading patterns and jaw movements during mastication in Macaca fascicularis: a bone-strain, electromyographic, and cineradiographic analysis. Am J Phys Anthropol 72, 287-314.

Hylander WL, Ravosa MR, Ross CF, et al. (1998) Mandibular corpus strain in primates: further evidence for a functional link between symphyseal fusion and jaw-adductor muscle force. Am J Phys Anthropol 107, 257-271.

Kupczik K, Dobson CA, Fagan MJ, et al. (2007) Assessing mechanical function of the zygomatic region in macaques: validation and sensitivity testing of finite element models. J Anat 210, 41-53.

Kupczik K, Dobson CA, Crompton RH, et al. (2009) Masticatory loading and bone adaptation in the supraorbital torus of developing macaques. Am J Phys Anthropol 139, 193-203.

Lieberman DE, Krovitz GE, Yates FW, et al. (2004) Effects of food processing on masticatory strain and craniofacial growth in a retrognathic face. J Hum Evol 46, 655-677.

Liu ZJ, Herring SW (2000) Bone surface strains and internal bony pressures at the jaw joint of the miniature pig during masticatory muscle contraction. Arch Oral Biol 45, 95-112.

Marinescu R, Daegling DJ, Rapoff AJ (2005) Finite-element modeling of the anthropoid mandible: the effects of altered boundary conditions. Anat Rec A 283, 3000-3309.

Mazzetta GV, Cisilino AP, Blanco RE (2004) Distribución de ensiones durante la mordida en la mandíbula de Carnotaurus sastrei Bonaparte, 1985 (Theropoda: Abelisauridae). Ameghiniana 41, 605-617.

Mazzetta GV, Cisilino AP, Blanco RE, et al. (2009) Cranial mechanics and functional interpretation of the horned carnivorous dinosaur Carnotaurus sastrei. J Vertebr Paleontol 29, 822-830.

McHenry C, Clausen P, Daniel WJT, et al. (2006) Biomechanics of the rostrum in crocodilians: a comparative analysis using finite-element modeling. Anat $\operatorname{Rec} A$ 288, 827-849.

Metzger KA, Daniel WJT, Ross CF (2005) Comparison of beam theory and finite-element analysis with in vivo bone strain data from the Alligator cranium. Anat $\operatorname{Rec} A$ 283, 331-348.

Moazen M, Curtis N, O'Higgins P, et al. (2009) Biomechanical assessment of evolutionary changes in the lepidosaurian skull. Proc Natl Acad Sci U S A 20, 8273-8277.

Molnar RE (1998) Mechanical factors in the design of the skull of Tyrannosaurus rex (Osborn, 1905). Gaia 15, 198-218. 
Moreno K, Wroe S, Clausen P, et al. (2008) Cranial performance in the Komodo dragon (Varanus komodoensis) as revealed by high-resolution 3-D finite element analysis. J Anat 212, 736-746.

Panagiotopoulou O, Curtis N, O'Higgins P, et al. (2010) Modelling subcortical bone in finite element analyses: a validation and sensitivity study in the macaque mandible. J Biomech 43 1603-1611.

Panagiotopoulou O, Kupczik K, Cobb SN (2011) The mechanical function of the periodontal ligament in the macaque mandible: a validation and sensitivity study using finite element analysis. J Anat 218, 75-86.

Pierce SE, Angielczyk KD, Rayfield EJ (2008) Patterns of morphospace occupation and mechanical performance in exant crocodilian skulls: a combined geometric morphometric and finite element modeling approach. J Morphol 269, 840-864.

Plotnick RE, Baumiller TZ (2000) Invention by evolution: functional analysis in paleobiology. In: Deep Time: Paleobiology's Perspective. (eds Erwin DH, Wing SL), pp. 305-323, Lawrence: The Paleontological Society.

Porro LB, Holliday CM, Anapol F, et al. (2011) Free body analysis, beam mechanics, and finite element modeling of the mandible of Alligator mississippiensis. J Morphol 272, 910-937.

Rafferty KL, Herring SW, Marshall CD (2003) Biomechanics of the rostrum and the role of facial sutures. J Morphol 257, 33-44.

Rayfield EJ (2011) Strain in the ostrich mandible during simulated pecking and validation of specimen-specific finite element models. J Anat 218, 47-58.

Rayfield EJ, Milner AC (2008) Establishing a framework for archosaur cranial mechanics. Paleobiology 34, 494-515.

Rayfield EJ, Norman DB, Horner CC, et al. (2001) Cranial design and function in a large theropod dinosaur. Nature 409, 1033-1037.

Rayfield EJ, Milner AC, Xuan VB, et al. (2007) Functional morphology of spinosaur 'crocodile-mimic' dinosaurs. J Vertebr Paleontol 27, 892-901.

Reed DA, Porro LB, Iriarte-Diaz J, et al. (2011) The impact of bone and suture material properties on mandibular function in Alligator mississippiensis: testing theoretical phenotypes with finite element analysis. J Anat 218, 59-74.

Ross CF (2001) In vivo function of the craniofacial haft: the interorbital 'pillar'. Am J Phys Anthropol 116, 108-139.

Ross CF, Dechow PC, Iriarte-Diaz J, et al. (2011) In vivo bone strain and finite-element modeling of the craniofacial haft in catarrhine primates. J Anat 218, 112-141.

Ross CF, Metzger KA (2004) Bone strain gradients and optimization in vertebrate skulls. Ann Anat 186, 387-396.

Ross CF, Patel BA, Slice DE, et al. (2005) Modeling masticatory muscle force in finite element analysis: sensitivity analysis using principal coordinate analysis. Anat Rec A 283, 288-299.

Ross CF, Berthaume MA, Dechow PC, et al. (2011) In vivo bone strain and finite-element modeling of the craniofacial haft in catarrhine primates. J Anat 218, 112-141.

Rybicki EF, Mills EJ, Turner AS, et al. (1977) In vivo and analytical studies of forces and moments in equine long bones. J Biomech 10, 701-705.

Schwenk K (2000) Tetrapod feeding in the context of vertebrate morphology. In: Feeding: Form, Function, and Evolution in Terrestrial Vertebrates. (ed. Schwenk K), pp. 3-20, San Diego: Academic Press.
Sinclair AG, Alexander RM (1987) Estimates of forces exerted by the jaw muscles of some reptiles. J Zool London 213, 107-115. Soons J, Herrel A, Genbrugge A, et al. (2010) Mechanical stress, fracture risk and beak evolution in Darwin's ground finches (Geospiza). Philos Trans R Soc Lond B Biol Sci 365, 1093-1098.

Strait DS, Wang Q, Dechow PC, et al. (2005) Modeling elastic properties in finite-element analysis: how much precision is needed to produce an accurate model? Anat $\operatorname{Rec} A \mathbf{2 8 3}$, 275-287.

Strait DS, Richmond BG, Spencer MA, et al. (2007) Masticatory biomechanics and its relevance to early hominid phylogeny: an examination of palatal thickness using finite-element analysis. J Hum Evol 52, 585-599.

Taylor MA (1992) Functional anatomy of the head of the large aquatic predator Rhomaleosaurus zetlandicus (Plesiosauria, Reptilia) from the Toarcian (Lower Jurassic) of Yorkshire, England. Philos Trans R Soc Lond B Biol Sci 335, 247-280.

Therrien F, Henderson DM, Ruff CB (2005) Bite me: biomechanical models of theropod mandibles and implications for feeding behaviour. In: The Carnivorous Dinosaurs. (ed. Carpenter K), pp. 179-237, Bloomington: Indiana University Press.

Van Drongelen W, Dullemeijer P (1982) The feeding apparatus of Caiman crocodilus; a functional-morphological study. Anat Anz 151, 337-366.

Verrue VL, Dermaut L, Verhegghe B (2001) Three-dimensional finite element modeling of a dog skull for the simulation of initial orthopaedic displacements. Eur J Orthod 232, 517-527.

Walmsley CW, Smits PD, Quayle MR, et al. (2013) Why the long face? The mechanics of mandibular symphysis proportions in crocodiles. PLoS ONE 8, e53878.

Weijs WA, De Jongh HJ (1977) Strain in mandibular alveolar bone during mastication in the rabbit. Arch Oral Biol 22, 667-675.

Weishampel DB (1995) Fossils, function and phylogeny. In: Functional Morphology in Vertebrate Paleontology. (ed. Thomason JJ), pp. 34-54, Cambridge: Cambridge University Press.

Williams SH, Wall CE, Vinyard CJ, et al. (2008) Symphyseal fusion in selenodont artiodactyls: new insights from in vivo and comparative data. In: Primate Craniofacial Function and Biology. (eds Vinyard CJ, Ravosa MR, Wall CE), pp. 39-61, New York: Springer.

Williams SH, Vinyard CJ, Wall CE, et al. (2009) Mandibular corpus bone strain in goats and alpacas: implications for understanding the biomechanics of mandibular form in selenodont artiodactyls. J Anat 214, 65-78.

Zapata U, Metzger K, Wang Q, et al. (2010) Material properties of mandibular cortical bone in the American alligator, Alligator mississippiensis. Bone 46, 860-867.

Zar JH (1999) Biostatistical Analysis. Upper Saddle River, NJ: Prentice Hall.

\section{Supporting Information}

Additional Supporting Information may be found in the online version of this article:

Video S1. Anterior view of deformation of the FEM amplified $10 \times$ during anterior biting.

Video S2. Anterior view of deformation of the FEM amplified $10 \times$ during posterior biting. 